University of Rhode Island

DigitalCommons@URI

Open Access Master's Theses

2011

\title{
THE FORMULATION OF NATURALLY OCCURING POLYPHENOLIC NUTRACEUTICAL AGENTS USING HOT-MELT EXTRUSION
}

Aditya Duvuri

University of Rhode Island, alchemist2410@gmail.com

Follow this and additional works at: https://digitalcommons.uri.edu/theses

\section{Recommended Citation}

Duvuri, Aditya, "THE FORMULATION OF NATURALLY OCCURING POLYPHENOLIC NUTRACEUTICAL AGENTS USING HOT-MELT EXTRUSION" (2011). Open Access Master's Theses. Paper 88.

https://digitalcommons.uri.edu/theses/88

This Thesis is brought to you for free and open access by DigitalCommons@URI. It has been accepted for inclusion in Open Access Master's Theses by an authorized administrator of DigitalCommons@URI. For more information, please contact digitalcommons-group@uri.edu. 
THE FORMULATION OF NATURALLY OCCURING

POLYPHENOLIC NUTRACEUTICAL AGENTS

USING HOT-MELT EXTRUSION

BY

ADITYA DUVURI

A THESIS SUBMITTED IN PARTIAL FULFILLMENT OF THE

REQUIREMENTS FOR THE DEGREE OF

MASTER OF SCIENCE

IN

BIOMEDICAL AND PHARMACEUTICAL SCIENCES

UNIVERSITY OF RHODE ISLAND 
MASTER OF SCIENCE IN BIOMEDICAL AND

PHARMACEUTICAL SCIENCES

OF

ADITYA DUVURI

APPROVED:

Thesis Committee:

Major Professor: Dr. David Worthen

Dr. Peng Wang

Dr. Navindra Seeram

Dr. Joan Lausier

Dr. Nasser. H. Zawia

DEAN OF THE GRADUATE SCHOOL

UNIVERSITY OF RHODE ISLAND

2011 


\begin{abstract}
The process of pharmaceutical formulation is constantly evolving as many new active compounds are being discovered in order to overcome the challenges associated with producing viable dosage forms to reach the target tissues by these compounds. Perhaps, the most important property for a drug to be effectively formulated into a dosage form is adequate miscibility and solubility which may be in an organic solvent vehicle but is mainly in water. Water solubility along with solubility in the body fluids is critical from the point of view of achieving the desired levels of the drug in the body for effective therapy against a disease condition. Since antiquity, active pharmaceutical compounds have been extracted from various natural sources such as plants and animals. In plants, compounds have been extracted from seeds, leaves, roots and fruits of the plant. The compounds resveratrol and quercetin belong to a class of compounds called polyphenols that have shown activity against a few disease conditions. These compounds have very low aqueous solubility and bioavailability and for achieving efficient therapeutic activity, may have to be formulated into more soluble and bioavailable dosage forms.
\end{abstract}

Hot-melt extrusion is a novel technique that has been explored for pharmaceutical manufacturing in recent years. Applied on a large scale in the plastics industry, hotmelt extrusion has been employed in improving the solubility of poorly soluble compounds by producing an amorphous solid solution of the drug in a medium such as an edible or erodible biocompatible polymer. It involves few processing steps and has a few advantages over other formulation methods used for pharmaceutical processing 
of poorly soluble active compounds. It was hypothesized that hot-melt extrusion would assist in improving the solubility and bioavailability upon oral delivery of the polyphenols in a viable dosage form. The polymer employed for the study was a novel polymeric solubilizer, Soluplus®. The study involved hot-melt extrusion of resveratrol and quercetin separately with Soluplus ${ }^{\circledR}$, described in Chapter 1, and analysis of the mixtures thus produced by using analytical techniques such as high performance liquid chromatography, differential scanning calorimetry and Fourier transform infra-red spectroscopy. Additionally, studies to determine the improvement in solubility and miscibility were conducted on the hot-melt extruded and physical mixtures of resveratrol and quercetin with Soluplus ${ }^{\circledR}$ and the properties of the mixtures upon formulation as films for topical delivery and formulation as tablets were studied. The dissolution characteristics of the hot-melt extruded mixture of resveratrol and Soluplus ${ }^{\circledR}$ were studied and compared to the dissolution characteristics of the corresponding physical mixture of resveratrol and Soluplus@. Lastly, the effect of forced degradation on the mixtures was studied and analyzed by high performance liquid chromatography. The methodology employed for this study has been described in Chapter 3. From the results and interpretations described in Chapter 4, it is observed that the hot-melt extruded mixtures of resveratrol and quercetin with Soluplus $₫$ might form stable, amorphous solid mixtures of the polyphenol in the polymer which was determined using differential scanning calorimetry. The high performance liquid chromatography analysis and the Fourier transform infra-red spectroscopy technique perhaps indicate that the content remains uniform even though different proportions of the active polyphenol were used with the polymer and that there is an intimate 
chemical interaction that may result in changes within the active polyphenol causing a change in the functional group characteristics of the polyphenols upon extrusion with Soluplus ${ }^{\circledR}$ as was observed through the Fourier transform infra-red spectroscopy technique. The formulation of the mixtures of resveratrol and quercetin with Soluplus ${ }^{\circledR}$ as films for topical delivery failed due to unstable films being produced and re-crystallization of the active polyphenol upon drying of the films. Tablet formulations of the hot-melt extruded and physical mixtures of both resveratrol and quercetin were produced and properties of the tablets were assessed. The tablets produced by the hot-melt extruded mixtures of quercetin were harder, less friable and had suitable disintegration times as compared to the tablets produced from the hotmelt extruded mixtures of resveratrol. From the dissolution studies performed, it is perhaps observed that a smaller particle size of the hot-melt extruded mixture of resveratrol and Soluplus ${ }^{\circledR}$ has a faster release rate as compared to the larger particle size fractions of the hot-melt extruded mixture of resveratrol and Soluplus®. Lastly, the forced degradation studies might indicate that the hot-melt extruded mixtures of resveratrol and quercetin with Soluplus ${ }^{\circledR}$ undergo degradation at elevated temperature conditions and perhaps, there is loss of the active polyphenols from the mixtures as a result. 


\section{ACKNOWLEDGMENTS}

I would like to extend my heartfelt love and gratitude towards my father Dr. Sudhakar Duvvuri, my mother Rupa Duvvuri, and my sister Tejasvi Duvuri whose continued love and support have been the biggest motivation for my graduate education and thesis. Importantly, this degree and the purpose of my education in the United States of America would never have been accomplished without my major advisor, Dr. David Worthen, who has not only been a mentor for my research in science but has also been an excellent teacher, a friend and a role model for a budding scientist like me.

I thank my committee members who have guided me and supported me in my quest to learn science at this esteemed University. I would also like to thank the College of Pharmacy at the University of Rhode Island for their financial support to my graduate education. I express my gratitude to all the professors from whom I have learnt in depth about pharmaceutical sciences. As an international student, I would like to thank the respective offices at the University of Rhode Island for their continuous support and assistance to all international students who are pursuing the subject of their choice at this University.

Lastly, I thank my lab colleagues, close friends and roommates here for all their help in my time of need. A special thank you also to my closest friend Anju Gupta and all my friends back in India for all their kind words and inspiration when I most needed them. 


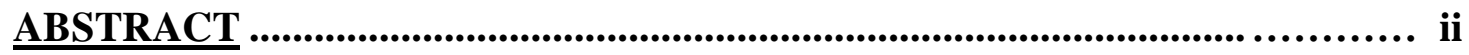

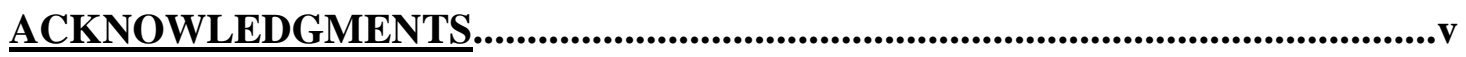

TABLE OF CONTENTS ............................................................................................... vi

LIST OF TABLES ............................................................................................ vii

LIST OF FIGURES ................................................................................................... vi

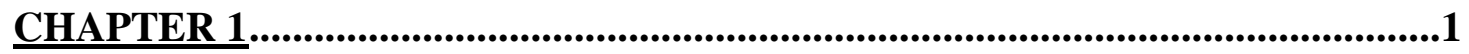

BACKGROUND

CHAPTER 2

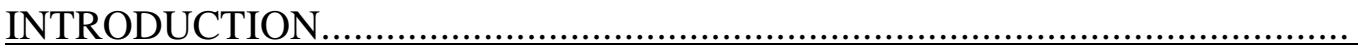

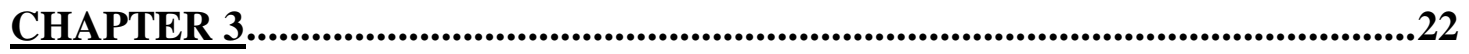

PRE-FORMULATION STUDIES AND THEIR SIGNIFICANCE ……………...

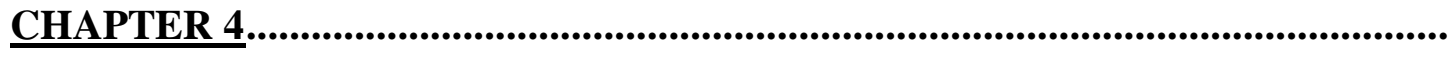

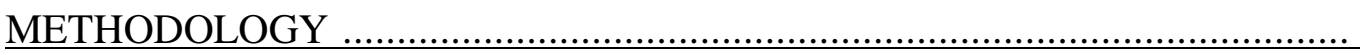

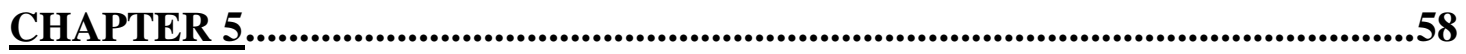

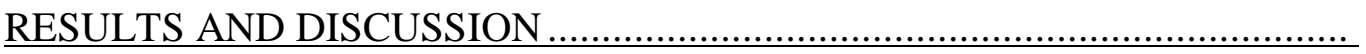

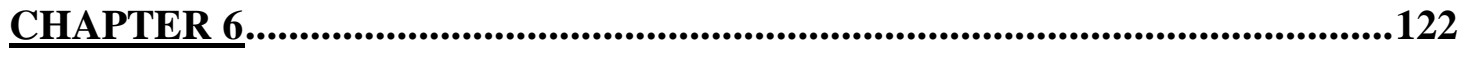

CONCLUSIONS ...................................................

BIBLIOGRAPHY ................................................................................................................124 


\section{LIST OF TABLES}

TABLE

PAGE

Table 1. A comparison of the molecular weights, melting temperatures,

aqueous solubility and bioavailability of polyphenolic compounds.... .3

Table 2. Prediction of miscibility based upon the difference in

solubility parameters

(the extent of interactions between the drug and the polymer).

Table 3. Important parameters of the hot-melt extrusion process

Table 4. Data of the concentration of the apparent solubilities of

resveratrol and quercetin in solutions of serial concentrations

of Soluplus® in water

59

Table 5. Data obtained for the concentrations of polyphenols in

different solutions of Soluplus $®$ in PBS and in water.

Table 6. Data obtained over 90 minutes for the mean cumulative

percentage $( \pm S D)$ of content released from the three capsules of

the hot-melt extruded mixture and similarly from the three capsules of the

corresponding physical mixture......................................... 72

Table 7. The average hardness of the tablets of the HME and

corresponding physical mixtures of resveratrol with Soluplus@

in kilograms.

Table 8. Data obtained for the hardness test performed on the

tablets produced from the physical mixtures and the hot-melt

extruded mixtures of quercetin and Soluplus ${ }^{\circledR}$ 
Table 9. Data showing the friability in percentage for the tablets produced from the physical mixture and the HME

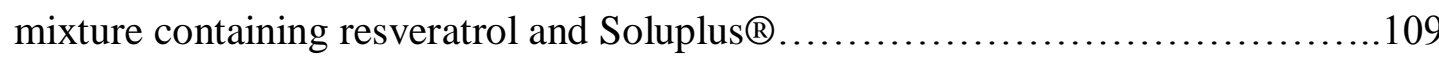
Table 10. Data obtained for the friability test on the tablets produced from the physical mixtures and the hot-melt extruded mixtures of quercetin and Soluplus ${ }^{\circledR}$

Table 11. Data obtained for the mean disintegration times $( \pm \mathrm{SD})$ of the tablets produced by the tablets obtained from the physical mixtures and HME mixtures of resveratrol and Soluplus $®$ and quercetin and Soluplus $₫$. 


\section{LIST OF FIGURES}

FIGURE

PAGE

Figure 1. Chemical structure of resveratrol....................................................2

Figure 2. Chemical structure of quercetin ........................................................2

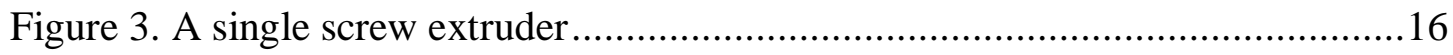

Figure 4. A twin screw extruder .............................................................. 18

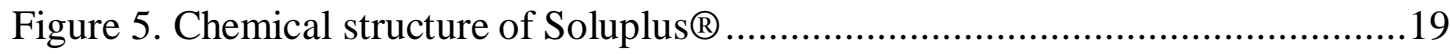

Figure 6. SDX Spray drying nozzle........................................24

Figure 7. An example of solvent evaporation............................... 25

Figure 8. White precipitate obtained on mixing solutions of polymer dissolved in the solvent and resveratrol dissolved

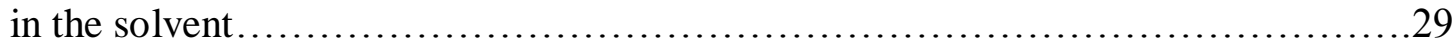

Figure 9. The white precipitate complex upon vacuum drying....................29

Figure 10. A comparison of the apparent solubilities of resveratrol

and quercetin in water and in solutions of Soluplus ${ }^{\circledR}$ in water.....................59

Figure 11. A chart showing the apparent solubilities of resveratrol and quercetin in water and in Soluplus ${ }^{\circledR}$ dissolved in PBS ..........................61

Figure 12. Multiple chromatogram view of $5 \%$ by weight of

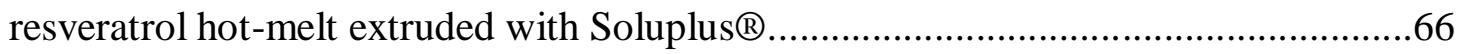


Figure 13. Multiple chromatogram view of two samples

of resveratrol hot-melt extruded with Soluplus ${ }^{\circledR}$

at a concentration of $5 \%$ by weight of resveratrol and

$2 \%$ by weight of resveratrol.

.66

Figure 14. Multiple chromatogram view of the hot-melt

extruded mixtures of resveratrol in Soluplus $®$ in the

concentrations of $2 \%$ and $5 \%$ by weight of resveratrol and

the physical mixture of $0.5 \%$ by weight of resveratrol in Soluplus $®$

Figure 15. Multiple chromatogram view of three samples of

Quercetin hot-melt extruded with Soluplus@ at a concentration

of $5 \%$ by weight of quercetin.

Figure 16. Multiple chromatogram view of two samples of

Quercetin hot-melt extruded with Soluplus $®$ at a concentration

of $5 \%$ and $20 \%$ by weight of Quercetin.

Figure 17. Multiple chromatogram view of a sample of Quercetin

physically mixed with Soluplus ${ }^{\circledR}$ at a concentration of $0.5 \%$ by weight

of Quercetin and a sample of Quercetin hot-melt extruded with Soluplus ${ }^{\circledR}$

at a concentration of $5 \%$ by weight of Quercetin

Figure 18. Multiple chromatogram view for a sample of Soluplus®

dissolved in $80 \%$ methanol and another sample of Soluplus®

dissolved in $95 \%$ methanol.

70 
Figure 19. Plot of mean percentage cumulative released $(\mathrm{SD} \pm 1)$ from capsules filled with HME mixture containing 5\% resveratrol by weight with a particle size between $180-250 \mu \mathrm{m}$ and Soluplus ${ }^{\circledR}$ and the corresponding physical mixture 73 Figure 20. Plot of mean percentage cumulative released $\left(\mathrm{SD}_{ \pm} 1\right)$ from capsules filled with HME mixture containing 5\% resveratrol by weight with a particle size between $250-425 \mu \mathrm{m}$ and Soluplus ${ }^{\circledR}$ and the corresponding physical mixture.

Figure 21. Plot of mean percentage cumulative released $(S D \pm 1)$ from capsules filled with HME mixtures containing 5\% resveratrol by weight with a particle size between $180-250 \mu \mathrm{m}$ and $250-425 \mu \mathrm{m}$ and Soluplus ${ }^{\circledR}$ and similarly, the corresponding physical mixtures...........75

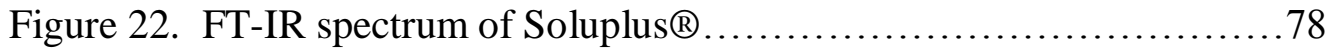
Figure 23. FT-IR spectrum of Resveratrol..............................79

Figure 24. FT-IR spectrum of Quercetin..............................81

Figure 25. FT-IR spectra of the hot-melt extruded mixtures of resveratrol and Soluplus ${ }^{\circledR}$

Figure 26. FT-IR spectra of a hot-melt extruded mixture of resveratrol in Soluplus ${ }^{\circledR} 20 \%$ by weight and a physical mixture of $20 \%$ resveratrol in Soluplus $®$.... .84

Figure 27. FT-IR spectra of Soluplus ${ }^{\circledR}$ and the HME mixture of $2 \%$ resveratrol by weight in Soluplus ${ }^{\circledR}$. .85 
Figure 28. FT-IR spectra of the HME mixtures of both weight

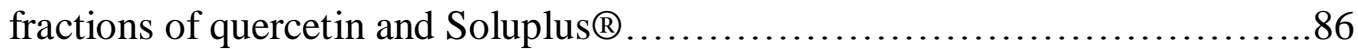

Figure 29. FT-IR spectra of the HME and physical mixture of

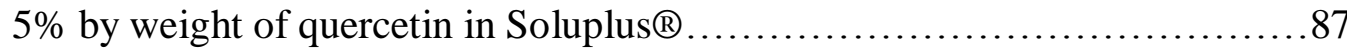

Figure 30. FT-IR spectra of quercetin HME and Soluplus ${ }^{\circledR}$ blank.............88

Figure 31. DSC thermograms of the physical mixtures of

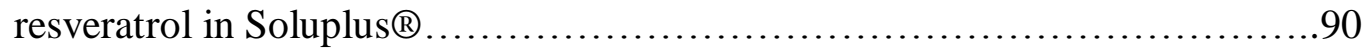

Figure 32. DSC thermogram of resveratrol............................ 92

Figure 33. DSC thermograms of the physical mixtures of

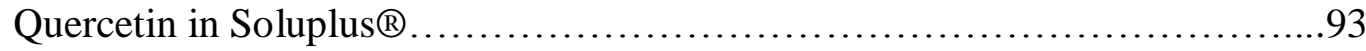

Figure 34. DSC thermogram of quercetin ...............................95

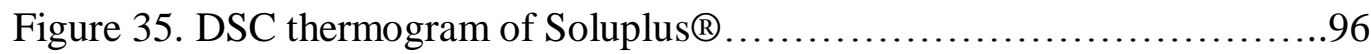

Figure 36. DSC thermograms of the hot-melt extruded and the physical mixture of $5 \%$ resveratrol by weight and Soluplus ${ }^{\circledR} \ldots \ldots \ldots \ldots \ldots \ldots . . . .67$

Figure 37. DSC thermograms of the hot-melt extruded and the physical mixture of $20 \%$ resveratrol by weight and Soluplus $® \ldots . . . \ldots \ldots . . .98$ Figure 38. DSC thermograms of the hot-melt extruded and the physical mixture of 5\% quercetin by weight and Soluplus ${ }^{\circledR} \ldots \ldots \ldots \ldots \ldots \ldots \ldots 100$ Figure 39. DSC thermograms of the hot-melt extruded and the physical mixture of $20 \%$ quercetin by weight and Soluplus ${ }^{\circledR} \ldots \ldots \ldots \ldots \ldots \ldots 101$ Figure 40. Tablets of HME mixtures containing 2\% resveratrol by weight and Soluplus $®$ 103 
Figure 41. Tablets of HME mixtures containing 5\% quercetin by weight and Soluplus ${ }$. .104

Figure 42. Multiple chromatogram view of the HME mixtures of $2 \%$ and $5 \%$ resveratrol by weight and Soluplus ${ }^{\circledR}$ after forced degradation

Figure 43. Chromatogram obtained for HME mixture containing $20 \%$ resveratrol by weight and Soluplus ${ }^{\circledR}$ after forced degradation

Figure 44. Multiple chromatogram view of the HME mixtures of $5 \%$ and $20 \%$ by weight of quercetin and Soluplus ${ }^{\circledR}$ after forced degradation

Figure 45. Multiple chromatogram view of $2 \%$ resveratrol by weight, HME with Soluplus $®$, before and after forced degradation

Figure 46. Multiple chromatogram view of $5 \%$ resveratrol by weight, HME with Soluplus ${ }^{\circledR}$, before and after forced degradation

Figure 47: Multiple chromatogram view of 5\% quercetin by weight

HME with Soluplus ${ }^{\circledR}$, before and after forced degradation ... 119

Figure 48: Multiple chromatogram view of $20 \%$ quercetin by weight

HME with Soluplus®, before and after forced degradation 


\section{Chapter 1}

\section{Background}

The field of drug therapy is dynamic and rapidly evolving. Active compounds have been extracted from myriad natural sources since antiquity (1). As numerous compounds have been discovered, the process of formulating them into an effective dosage form to reach target tissues becomes an equally challenging task. The most important characteristics for a drug to be formulated into an effective dosage form are adequate miscibility and solubility in a desired solvent vehicle such as an aqueous media and in the aqueous body fluids and tissues of humans and animals (2). In the case of delivery to a mammal, advances in drug discovery and high-throughput screening have brought forth many active compounds with low solubility and permeability characteristics. One class of such molecules is polyphenols, which occurs in and have been extracted from the seeds, roots, fruits and leaves of certain plants (3). Phenolic acids which include polymeric structures, such as hydrolyzable tannins, lignans, stilbenes, and flavonoids are some of the most important classes of polyphenols. Flavonoids include flavonols (e.g. quercetin and kaempferol, which are commonly found in food), flavones, isoflavones, flavanones, anthocyanidins pigments responsible for the colour of most fruits), flavanols, and catechins-monomers and proanthocyanidins_-polymers, known as condensed tannins.

Polyphenols (PPs) may be classified into simple phenols/phenolic acids (e.g., p-cresol, 3-ethylphenol, vanillic, gallic, ellagic acids, hydroquinone), hydroxycinnamic acid derivatives (e.g., p-coumaric, caffeic, ferulic, sinapic acids), flavonoids, and tannins in 
order to describe their chemical structures and functions, such as antimicrobial activity. Due to their activity against micro-organisms, phenolic acids can be considered as good anti-microbial agents. Resveratrol and quercetin belong to the general class of polyphenols and their biological activity against various conditions has been documented. For the current task of studying the formulation characteristics of polyphenols, two compounds were selected, namely, resveratrol and quercetin.

Resveratrol has been extracted from Japanese knotweed and other sources and marketed as a nutritional supplement (4). Chemically, it is a 3,5,4'-trihydroxy-transstilbene (5), a compound found in red wine that has been assessed for its anti-aging (6) and ant-diabetic effects(7). Quercetin is a plant-derived flavonoid that is claimed to possess anti-inflammatory and anti-oxidant properties $(8,9)$. It is also known as meletin or sophoretin (10) and is the aglycone form of the compound quercitrin that is found in citrus fruits and buckwheat (11).

These compounds, resveratrol and quercetin have very low solubility in water $(0.03 \mathrm{~g} / \mathrm{l}$ for Resveratrol) (12) and other commonly utilized organic solvents. The structures of these compounds are shown in the figures below.

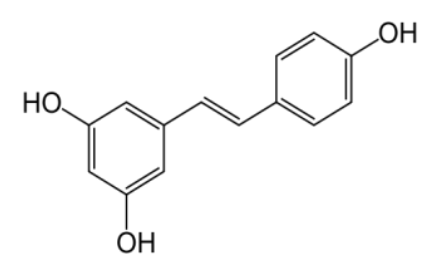

Figure 1: Chemical structure of resveratrol

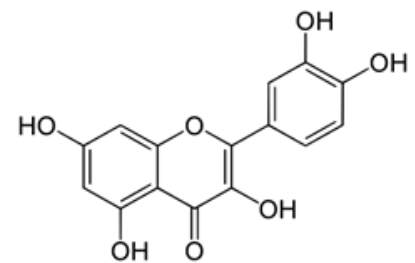

Figure 2: Chemical structure of quercetin 
From the figures 1 and 2 (13), it is evident that these compounds have some similar structural properties. They are polyphenolic aromatics, differing in ring skeleton structure, ring substitution and connectivity and in the number of associated phenol groups. Following is a table that describes the melting points and the water solubility of these compounds.

\begin{tabular}{|l|l|l|l|l|}
\hline Compound & Molecular & Melting point & $\begin{array}{l}\text { Aqueous } \\
\text { solubility }\end{array}$ & Bioavailability \\
\hline Resveratrol & 228.2 & 254 & 30 & Low \\
\hline Quercetin & 302.2 & 316 & $>30$ & $\sim 5$ \\
\hline Bisphenol A & 228.3 & 158 & 300 & $\sim 5$ \\
\hline
\end{tabular}

Table 1: A comparison of the molecular weights, melting temperatures, aqueous solubility and bioavailability of polyphenolic compounds

In the table above Bisphenol A, a compound commonly used as in polycarbonate manufacturing and epoxy resins has been listed for comparison. It possesses toxic effects on the body despite its relatively low bioavailability. As can be seen from the table, it has a molecular weight similar to resveratrol but has higher water solubility than both resveratrol and quercetin (14).

Currently, there is very little knowledge about the absorption, bioavailability, biodistribution and metabolism of polyphenols. Studies have indicated that there exists a common pathway by which most polyphenols are metabolized. The non-conjugated forms, aglycones, are generally absorbed intact from the digestive tract. The ester forms, glycosides and polymers, must undergo hydrolysis before being absorbed. 
Certain oral and intestinal microorganisms degrade polyphenols into aglycones and, occasionally, produce simple aromatic acids. Once absorbed, polyphenols are conjugated into methylated, glucuronidated or sulphated derivatives.

This metabolic process is also common to many xenobiotics. The above mentioned mechanisms are efficient in metabolizing polyphenols to the extent that the aglycones are nearly absent or present in very low concentrations in the blood after consumption of nutritional doses of these compounds. Besides other tissues, polyphenols are mainly detected in the mucosae of the digestive tract and primarily the oral mucosa after ingestion. Isoflavones and gallic acid are best absorbed in the gastrointestinal tract, followed by flavanones, catechins and quercetin glycosides whereas proanthocyanidins, anthocyanins and galloylated catechins are poorly absorbed. All polyphenols are excreted primarily in the urine and bile. The bioavailability for resveratrol and ellagic acid has been listed as low in literature, while that of quercetin has been estimated to lie between 2 to $4 \%(3,15)$

Oral formulations of polyphenols:

Certain polyphenolic compounds obtained from tea (Camellia sinensis, fam. Theaceae), catechins and theaflavins, have been reported to prevent tooth decay and oral cancer. A study was conducted in order to determine the effectiveness of green tea leaves and black tea extract as formulations for delivering catechins and theaflavins to the oral cavity. In this study, brewed black tea (2 $\mathrm{g}$ of black tea leaves in $100 \mathrm{ml})$ and green tea leaves $(2 \mathrm{~g})$ were held in the mouth for $2-5$ minutes. After rinsing the mouth, high concentrations of catechins (Cmax 131.0 -2.2 M) and theaflavins (Cmax 1.8-0.6 
M) were observed in saliva in the first hour. The overall kinetic profile was similar for both compounds with t1/2 equaling 25-44 minutes and 49-76 minutes for catechins and theaflavins respectively. However, a significant interindividual variation in the peak levels of catechins and theaflavins was observed (average coefficient of variation in $\mathrm{t} 1 / 2$ was $23.4 \%$ ). There were five unidentified peaks observed in the saliva after black tea treatment in addition to the parent catechin and theaflavin peaks. Theaflavin gallates underwent hydrolysis, apparently due to the salivary esterases, and this was observed both, in vitro and in vivo. The results indicated that tea leaves could be used as a convenient, slow-release source of catechins and theaflavins and provided information that would be helpful for the possible use of tea in the prevention of oral cancer and dental caries (16).

About 4000 compounds have been recognized as belonging to the class of compounds generally referred to as flavonoids. These poorly soluble compounds are rapidly degraded and metabolized in the human body, but possess potential in the prevention and therapy of major chronic diseases, including cardiovascular and neurodegenerative diseases and cancer. In recent years a number of flavanols such as quercetin, myricetin and catechin, and isoflavones such as genistein and daidzein have been confirmed to possess strong anti-oxidant, anti-inflammatory, anti-proliferative and anti-aging activities. The oral delivery of flavonoids can be markedly improved by incorporation into lipidic or polymer-based nanoparticles as they can protect the drug from degradation in the gastrointestinal tract. They possess a unique absorption mechanism through the lymphatic system and thus shield the drug from first-pass metabolism in the liver. Other formulations for the parenteral and oral routes might be 
explored for improved delivery, perhaps, sustained release and targeted delivery can be achieved (17).

Arsenic is a toxic substance which causes oxidative damage to the liver leading to hepatic fibrosis. A study was conducted to evaluate the therapeutic efficacy of liposomal and nanocapsulated polyphenolic anti-oxidant quercetin (QC) in treating arsenic induced hepatic oxidative stress and the subsequent fibrosis associated upregulation of its gene expression and plasma TGF beta (transforming growth factor beta) in a rat model. When a single dose of arsenic (sodium arsenite-NaAsO(2), 13 $\mathrm{mg} / \mathrm{kgb} . \mathrm{wt}$ ) is administered orally, the generation of reactive oxygen species (ROS) and arsenic accumulation in liver, followed by hepatotoxicity and a decrease in the hepatic plasma membrane microviscosity and anti-oxidant enzyme levels in the liver occurred. An increase in the gene expression, plasma TGF ss (from normal value $75.2+/-8.67 \mathrm{ng} / \mathrm{ml}$ to $196.2+/-12.07 \mathrm{ng} / \mathrm{ml}$ ) of arsenic occurred in the liver due to fibrosis and release of cytochrome $\mathrm{c}$ in cytoplasm was also observed. Two vesicular delivery systems were formulated with QC and among them, polylactide nanocapsules showed a promising result compared to liposomal delivery system, in controlling arsenic induced alteration of the mentioned parameters. Arsenic was administered orally to the test rats and an hour later, a single dose of $0.5 \mathrm{ml}$ of nanocapsulated QC suspension (QC $2.71 \mathrm{mg} / \mathrm{kg}$ by weight) was injected to the rats. It was observed that, the liver was protected from arsenic induced deterioration of anti-oxidant levels as well as oxidative stress associated gene expression of the liver. A histopathological examination was further carried out, which confirmed the pathological improvement in the liver. Thus, the nanocapsulated plant origin flavonoidal compound may be 
considered as a potent formulation in negating arsenic induced up-regulation of gene expression due to liver fibrosis, based on its property of complete protection against oxidative attack in hepatic cells of rat liver (18).

Resveratrol has been shown to be effective against certain skin disorders. Upon oral delivery, it is observed that resveratrol has very poor bioavailability and hence, systemic delivery of resveratrol by the oral route is a challenging task. Another polyphenolic formulation study conducted, explored the viability of resveratrol for topical delivery through the skin. The delivery systems were prepared in saturated solutions of soyabean oil, $\mathrm{pH} 6$ buffer, $\mathrm{pH} 8$ buffer and other suitable solutions and their effects on the in vitro permeation and skin deposition of resveratrol were studied. The observations made for the factors stated above displayed the following trend: $\mathrm{pH}$ 6 buffer=pH 8 buffer>10\% glycerol formal in $\mathrm{pH} 6$ buffer>pH 9.9 buffer>pH 10.8 buffer>soybean oil. Additionally, it was noted that a linear relationship could be established between the permeability co-efficient and the drug accumulation in the skin reservoir. The primary barriers in the delivery of non-ionic resveratrol were the dermis and the epidermis. The stratum corneum and viable skin served as obstacles to the delivery of anionic resveratrol.

Numerous prototype hydrogel systems were also studied as vehicles for the delivery of resveratrol. However, in this case, the viscosity and not the polarity was the controlling factor for permeation and deposition in the skin. The hydrogel caused no erythema or disruption of the stratum corneum as resveratrol has a good safety profile. From this study, it was concluded that topical delivery of resveratrol may serve as a 
potent route for its desired therapeutic effects. It was also, one of the first studies to report the permeation profiles for topically applied resveratrol (19).

As of today, only a few oral polyphenol products are commercially available in the market. For example, Walgreens pharmacy has a dietary supplement of resveratrol available in the form of capsules (20). Since there is very little knowledge about the metabolism of polyphenols, research is being conducted widely to assess new dosage forms and routes of administration. Some scientists have investigated injectable sustained release microparticles of curcumin, a chemical entity found in the popular Indian spice turmeric (21). Analogous formulations might be considered for polyphenols, although they would have the same disadvantages of other parenterals.

Thus, there exists a need for oral /solid formulations and dosage forms with improved solubility and bioavailability characteristics for these polyphenols, particularly for oral delivery via tablets or capsules. An important goal would be to increase the dissolution rate and extent and overcome gastrointestinal metabolism by delivering stable formulations with immediate and perhaps sustained effects. The critical idea in formulations is to produce stable, durable and high quality dosage forms which maintain their activity under conditions of storage for a given period of time. It is proposed that hot-melt extrusion of polyphenols and edible polymers will lead to the development of these dosage forms. 


\section{Chapter 2}

\section{Introduction}

In recent years, novel techniques have been explored for pharmaceutical manufacturing. One such method is hot-melt extrusion. Hot-melt extrusion is a process that has been applied on a large scale in the plastics industry. It has been confirmed recently that hot-melt extrusion is feasible in the pharmaceutical industry as a method to produce drug delivery systems. Hot- melt extrusion is applicable in improving the solubility of such compounds by producing an amorphous solid solution of the drug in a medium such as an edible or erodible biocompatible polymer. This method has a number of advantages over other established formulation approaches, such as spray drying and co - precipitation. These include: the absence of solvents, few processing steps; and improved bioavailability as compared to existing dosage forms. It is also a continuous process, and thus requires a short period of time to be completed while producing large amounts of material rapidly. Dosage forms produced by hot-melt extrusion are complex mixtures of active medicaments and functional excipients. Most often these include the active pharmaceutical ingredient, a medium such as a polymer and a co-polymer and a plasticizer which may be employed at times. The actual formulation process may involve heating, and softening of a mixture of drug and polymer followed by the extrusion of this molten mixture through a die.

The method of hot-melt extrusion can be combined with traditional methods of formulation to produce granules, pellets, capsules, and transdermal and transmucosal systems. This demonstrates the value of this technique as an effective and versatile 
tool in pharmaceutical processing and the production of safe, effective, and versatile drug delivery systems. The equipment involved in the manufacturing process can either be a single screw or a twin screw extruder $(22,23)$.

For formulating a dosage form using hot-melt extrusion, the raw materials that may be required include a drug, a biocompatible polymer, plasticizers, surfactants, and antioxidants. Due to the advances in high-throughput screening techniques and combinatorial chemistry, a number of active molecules discovered in the recent years have very low aqueous solubility. The aim of hot-melt extrusion formulation is to develop a dosage form that contains a stable amorphous solid solution of the drug in a polymer. According to the biopharmaceutics classification system developed by Amidon et al (24), drugs can be classified into:

a) BCS Class 1 - soluble and permeable

b) BCS Class 2 - permeable but poorly soluble

c) BCS Class 3 - soluble but poorly permeable

d) BCS Class 4 - poorly soluble and poorly permeable

The drugs belonging to classes 2 and 4 may have high molecular weights and exhibit poor bioavailability. These active substances might be formulated using hot-melt extrusions into various dosage forms to improve their bioavailability. This occurs due to a decrease in drug particle size or the formation of a molecular dispersion and an increase in the dissolution surface area as the drug is molecularly dispersed within the polymer (25). 
The second most important component required in formulation is the polymer. There are several polymers which are commercially available and used frequently in the formulation of myriad dosage forms. These include: Eudragit polymers (EPO, L-10055, and others), hydroxy propyl methyl cellulose (HPMCAS-LF); Pharmacoat polymers, and Kollidon polymers, such as Kollidon VA-64. A combination of these polymers may also be chosen in certain processes. Different polymer ratios and drugpolymer ratios may be selected based on the properties of the polymers and the dosage form to be manufactured (26).

In some formulations it may be essential to utilize an antioxidant or a plasticizer. Plasticizers reduce the glass transition temperatures of polymers by embedding themselves between the chains of polymers, thus spacing them apart and promoting flow. An antioxidant such as butylated hydroxyanisole (BHA) or propyl gallate (PG) may be incorporated into the formulation to prevent oxidation of the active substance or the polymer, which may otherwise result in decreased stability under storage conditions. The determination of properties such as the solubility of a drug in the polymer and the glass transition temperature of the polymer is an important step before the actual process of hot-melt formulation (27).

The assessment of solubility and glass transition temperatures

The active drug molecule that is to be formulated by hot-melt extrusion must have adequate miscibility with the polymer in which it is dispersed. It is generally believed that for adequate stability of the amorphous drug present in the final solid dispersion system, the formulation of a single phase amorphous solid solution is essential. Thus, 
there should not be any re-crystallization of the amorphous drug within the solid dispersion. This can be greatly reduced by increasing the glass transition temperature of the polymer thereby decreasing the molecular drug mobility, promoting interactions between the polymer and the drug, rather than promoting drug-drug interactions and recrystallization and by interference with the molecular recognition process for recrystallization. This would only occur if there is adequate mixing between the drug and the polymer. If phase separation occurs or if the drug and polymer are not miscible in their molten states, drug-rich amorphous phases or polymer-rich phases will be present in the solid dispersion formed upon solidification. In this case, even slight fluctuations in temperature or concentrations can trigger re-crystallization of the metastable amorphous drug present in the system. (25)

Many techniques have been suggested and have been employed for determining the miscibility of the drug with the polymer. The extent of the interactions between drug and polymer can be defined as the difference in solubility parameter between solute and solvent. The general assumption made here is that the polymer is the solvent and the drug is the solute. They interact to different extents resulting in varied drug polymer miscibility. For example, as reported in the miscibility study conducted by Greenhalgh, et al, (28) on molten drugs and excipients possessing various solubility parameters, an increase in the degree of immiscibility occurs with an increase in the difference of solubility parameter between the drug and carrier. The difference in solubility parameters are indictors of the potential miscibility between the drug and the polymer in solid dispersions. 
This can be predicted based upon a few critical values as explained by the following table:

\begin{tabular}{|c|l|}
\hline Difference in solubility parameter & Likelihood of Miscibility \\
between drug and polymer & \\
\hline$<7.5$ & Likely completely miscible \\
\hline $7.5-15.0$ & Likely partially miscible \\
\hline$>15.9$ & \\
\hline & Likely totally miscible \\
\hline
\end{tabular}

Table 2: Prediction of miscibility based upon the difference in solubility parameters (the extent of interactions between the drug and the polymer).

Another theory for determining the drug-polymer miscibility in amorphous solid dispersions is the Flory-Huggins lattice model. It accounts for a number of factors such as specific drug-polymer interactions, solute-solvent size disparity, and excess entropy of mixing. This theory suggests that the excess free energy of mixing is a function of the molar fraction (n), the volume fraction $(\phi)$ and the drug-polymer interaction parameter $(\chi)$.

Thus, by measuring the free energy of mixing, the miscibility of the drug with the polymer can be predicted. This can be carried out by using the following equation

$$
\begin{aligned}
& \underline{\Delta \mathrm{G}}=\mathrm{n} 2 \operatorname{In} \phi 2+\mathrm{n} 1 \operatorname{In} \phi 1+\mathrm{n} 2 \phi 1 \chi \quad \text { Equation } 1 \\
& \text { RT }
\end{aligned}
$$


Negative $\Delta \mathrm{G}$ values indicate stronger interactions between a drug and the polymer. This occurs due to lower interaction parameters and thus, there would be a stronger driving force to form a one- phase solution system. A critical value has also been suggested for the Gibbs free energy of mixing. A value higher than 0.5 indicates that the solute and solvent are completely immiscible or partially miscible depending upon the ratio of their molecular weights; a value of 0.5 or less would indicate complete miscibility and a negative value indicates a high degree of specific interactions between the drug and the polymer. Thus, there may be bond formation such as ionic or hydrogen-bond interactions between the drug and the polymer. (25)

The determination of glass transition temperature of the polymer is another important step to be performed before beginning the process of formulation. Higher glass transition temperatures would prevent re-crystallization of the amorphous drug in a solid dispersion and thus improve stability.

The most common method of measuring the glass transition temperature is by utilizing the differential scanning calorimeter (DSC). It is a thermo-analytical technique in which the difference in the amount of heat required to increase the temperature of a sample and a reference are compared. The sample and the reference are generally maintained at the same temperature. The basic underlying principle of the DSC is that a sample requires either more heat or less heat depending upon the transition it undergoes. For example, if, the sample is a solid that melts to form a liquid, it would require more heat to remain at the same temperature as the reference as it melts. In case the sample undergoes crystallization (an exothermic process), less heat is required to raise the temperature of the sample. Thus, even subtle phase changes such 
as glass transitions can be measured using DSC. The result of the DSC is obtained in the form of a curve of heat flux versus temperature or versus time (29).

The equipment used in a typical HME process can be described as having a platform that supports the drive system followed by an extrusion barrel, and a rotating screw arranged on a screw shaft leading into an extrusion die that determines the shape of the final product. The process parameters are generally controlled with the help of an electronic control unit. The extrusion drive system consists of various parts such as a motor, a gearbox, and linkage and thrust bearings. The extruders are classified into two types based on the arrangement of the rotating screw and barrel. Simple single screw extruders consist of a single rotating screw within a stationary extrusion barrel, whereas the twin screw systems make use of a co-rotating or counter-rotating screw within the extrusion barrel.

A simple single screw extruder consists of a single rotating screw inside the extrusion barrel. It is divided into three zones based on the function of each part. These are known as the feed zone, compression zone, and the metering zone. Variable pressure is generated along the length of the screw due to differences in the depth and/or pitch of the screw in each zone. Thus, low pressure is maintained in the feed zone to assist in continuous feeding of powdered and granulated materials through the hopper and allows for gentle mixing of the excipients and the active ingredient. The feed zone is followed by the compression zone, the primary function of which is to melt, homogenize and compress the extrudate so that it reaches the metering zone in a form suitable for extrusion. The materials undergo a high degree of mixing and compression in this zone which is achieved by decreasing the screw pitch and/or the flight depth, 
resulting in a gradual increase in pressure along the length of the compression zone. The metering zone is the final section that stabilizes the pulsating flow of the matrix, thus ensuring uniform thickness of the extruded product. Screw flight depth and pitch remain constant in this zone which maintains continuous high pressure to ensure a uniform delivery rate of molten material through the extrusion die and, hence, a uniform product.

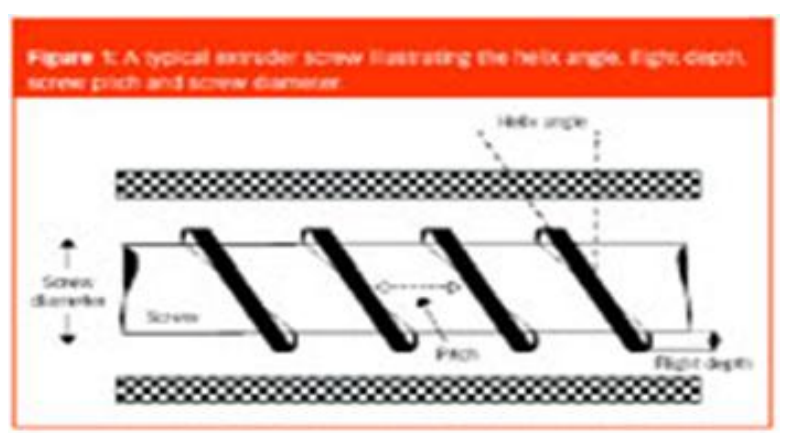

Figure 3: A single screw extruder (30)

The screw extruder consists of three different parts:

1) A conveying system for material transport and mixing

2) A die system for forming the desired shape; and

3) Downstream auxiliary equipment for cooling, cutting and/or collecting the finished product. 
Single screw devices operate by a relatively simple process and are generally not the preferred approach for the production of pharmaceutical formulations due to slower throughput and inferior mixing. On the other hand, the twin screw extruder is versatile, allows process manipulation and optimization, and the ability to accommodate various pharmaceutical formulations. In a twin screw extruder, rotation of the screws inside the extruder barrel may either be in the same direction (corotation) or in the opposite direction (counter-rotation). The directions of rotation, although opposite, are considered equivalent from a processing perspective. Another important design characteristic is the presence of intermeshing and non- intermeshing screws. Of the two, the intermeshing screws are preferred because of the greater degree of conveying achievable and the shorter residence times. By making use of forward conveying elements, reverse conveying elements, kneading blocks and other designs, the configuration of the screws may be varied to achieve particular mixing characteristics. The twin screw extruder is a favorable option as it offers a rapid and continuous process with a much better mixing capability as compared to the single screw extruder. Additionally, the melting process is more stable, the residence times are shorter and output is significantly greater. It is a practical process and has the potential to combine separate batch operations into a single continuous process, thus increasing manufacturing efficiency (30). 


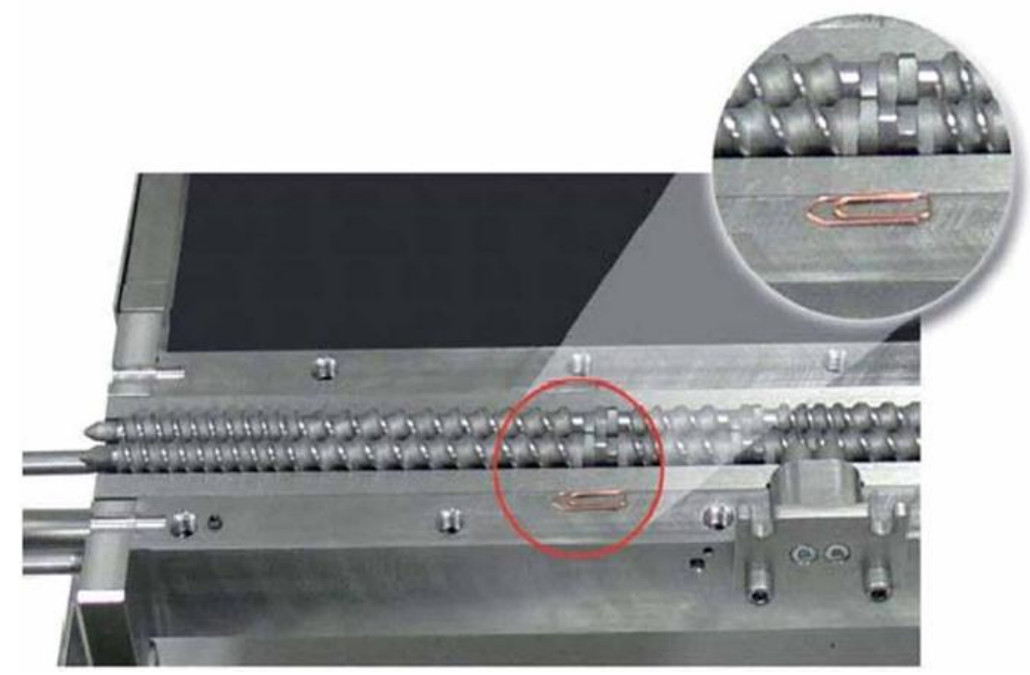

Figure 4: A twin screw extruder (31)

Thus, summarizing the hot-melt extrusion formulation process, it can be stated that the mixture of active ingredient, polymer and excipients is loaded into the feeder of the extrusion device where it undergoes compression under high temperature and pressure to give a molten mass of the materials that have been completely mixed together. On extrusion through the mold or die, and upon sufficient cooling, an amorphous, solid solution of the drug in polymer is obtained.

Since hot-melt extrusion assisted in producing stable and amorphous, solid solutions of the drug in a polymer, it was hypothesized that the formulation of polyphenolic nutraceuticals using this technique would result in producing more soluble and bioavailable dosage forms of the polyphenolic nutraceuticals. Additionally in the method of formulation, a novel polymeric solubilizer, Soluplus® was employed. It was hypothesized that this polymer would improve the solubility of the polyphenols and assist in producing stable and amorphous dispersions of the drug in Soluplus@. 
Active constituents used:

Resveratrol and quercetin.

Polymer used for the study:

Soluplus ${ }^{\circledR}$ (CAS-No 402932-23-4; HS 3905.99.00.0) is a new polymeric solubilizer developed in a similar fashion to the povidones (Kollidon ${ }^{\circledR}$ ) by the BASF group. It has an amphiphilic chemical structure and was produced particularly for solid solutions produced by hot-melt extrusion. It is capable of acting as a matrix polymer for solid solutions and is claimed to solubilize poorly soluble drugs in aqueous media. It can also increase the bioavailability of poorly soluble drugs. Chemically, it is a polyvinyl caprolactam-polyvinyl acetate-polyethylene glycol graft co-polymer (figure shown below). The molecular weight of Soluplus® lies in the range of 90,000$140,000 \mathrm{~g} / \mathrm{mol}$. With a critical micelle concentration (CMC) of $7.6 \mathrm{mg} / \mathrm{l}$ in water and a glass transition temperature $(\mathrm{Tg})$ of close to $70^{\circ} \mathrm{C}$, Soluplus ${ }^{\circledR}$ can be efficiently employed for the process of hot-melt extrusion as it requires less heating than other commonly used polymers. It has significant solubility characteristics and is soluble in water and in a few organic solvents in the range of $45-50 \%$ by weight. 


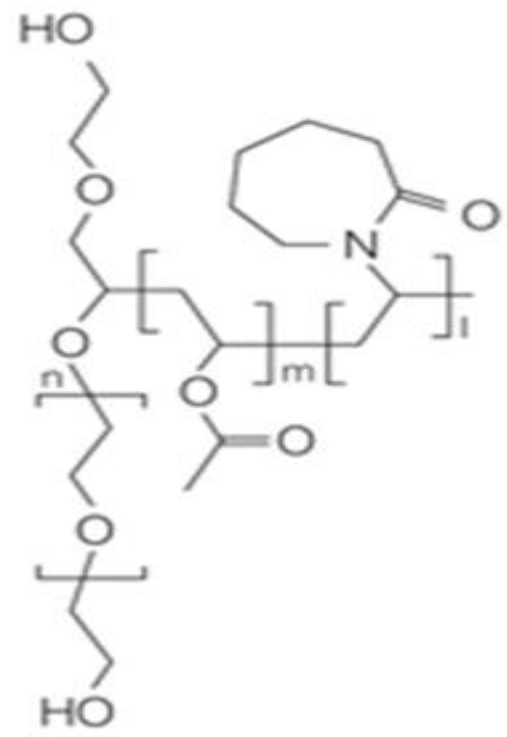

Figure 5: Chemical structure of Soluplus ${ }^{\circledR}$ (32)

Soluplus ${ }^{\circledR}$ is useful as a polymer in numerous pharmaceutical dosage forms. It can be used as binder in wet granulation or dry granulation processes for tablet manufacturing. Active pharmaceutical ingredients (APIs) can be incorporated into concentrated solutions of Soluplus ${ }^{\circledR}$ in a suitable solvent and can be prepared as films for topical application. Amorphous solid solutions of Soluplus® can be formulated into hard gelatin capsules by reduction of the size of the extrudates by milling processes, followed by capsule filling. Another useful formulation technique that can be effectively employed using Soluplus® is Spray -drying/ Spray-coating of the polymer and the poorly soluble API. For this purpose, Soluplus ${ }^{\circledR}$ and the API are dissolved in an appropriate organic solvent and can then be sprayed onto the surface of drug free pellets or sugar pellets (32).

In this study, Soluplus ${ }^{\circledR}$ was selected and evaluated as a melt extrusion polymer for formulating resveratrol and quercetin. 
The extrusion of resveratrol and Soluplus ${ }^{\circledR}$ and quercetin and Soluplus ${ }^{\circledR}$ was carried out using a twin screw extruder at Foster Corporation. In the process of extrusion, different conditions of temperature and pressure were used for different mixtures and the temperature again differed from one zone to another within the extruder. These are very important process parameters and form the backbone of the formulation process. These have been shown in the table below:

\begin{tabular}{|c|c|c|}
\hline Parameter & $\begin{array}{l}\text { Resveratrol and } \\
\text { Soluplus }{ }^{\circledR}\end{array}$ & Quercetin and Soluplus ${ }^{\circledR}$ \\
\hline Zone 1 & $72.3^{\circ} \mathrm{C}$ & $73.4^{\circ} \mathrm{C}$ \\
\hline Zone 2 & $130.0^{\circ} \mathrm{C}$ & $129.8^{\circ} \mathrm{C}$ \\
\hline Zone 3 & $155.0^{\circ} \mathrm{C}$ & $154.9^{\circ} \mathrm{C}$ \\
\hline Zone 4 & $155.2^{\circ} \mathrm{C}$ & $154.7^{\circ} \mathrm{C}$ \\
\hline Zone 5 & $164.8^{\circ} \mathrm{C}$ & $165.0^{\circ} \mathrm{C}$ \\
\hline Zone 6 & $164.9^{\circ} \mathrm{C}$ & $164.9^{\circ} \mathrm{C}$ \\
\hline $\begin{array}{l}\text { Melting } \\
\text { temperature }\end{array}$ & $164.5^{\circ} \mathrm{C}$ & $165.7^{\circ} \mathrm{C}$ \\
\hline $\begin{array}{l}\text { Screw speed } \\
(\mathrm{rpm})\end{array}$ & 49.7 & 49.5 \\
\hline Torque $(\mathrm{Nm})$ & 98.0 & 55.4 \\
\hline Pressure (bar) & Medium & Low \\
\hline $\begin{array}{l}\text { Feed rate } \\
(\mathrm{lb} / \mathrm{hr})\end{array}$ & 2.5 & 2.5 \\
\hline
\end{tabular}

Table 3: Important parameters of the hot-melt extrusion process 


\section{Chapter 3}

Pre-formulation studies and their significance

\section{Abstract:}

One of the most critical aspects of pharmaceutical formulation is adequate solubility of the active constituents in water or a suitable solvent vehicle. The polyphenolic compounds have displayed biological activity against many types of specific conditions and disease processes, but the area of concern is their delivery using a convenient dosage form due to their poor aqueous solubility and low bioavailability. Various techniques have been established in the past for the formulation of poorly soluble compounds. These include solvent evaporation/solvent casting, spray drying technology, co-precipitation and preparation of amorphous solid dispersions of the drug in a polymer. Before initiating a formulation process, certain studies are carried out on the constituents and ingredients to be used in the pharmaceutical process. These studies assist in analyzing and determining the characteristic properties of the raw materials and the critical parameters involved in the process that affect these compounds such as solubility, stability and chemical compatibility. These are known as pre-formulation studies. Before hot-melt extrusion could be selected as the method of choice for pharmaceutical processing of these polyphenols, individual tests with solvent evaporation were conducted in order to assess their feasibility for the extrusion process. Formulations of resveratrol and quercetin were tested using solvent evaporation with Kollidon VA64 and Lutrol F-68 as the polymer-surfactant mixture in a variety of solvents. The studies revealed that the polyphenols could not be 
formulated using solvent evaporation, as an insoluble precipitate of the test polymer and the polyphenols was obtained on addition of the components dissolved in a common solvent. The same result was noted when Soluplus® was used as the polymer. Thus, solvent based formulation processes, such as spray drying and solvent evaporation, were ruled out, and melt extrusion was identified as a possible alternative formulation process.

Introduction:

The chosen polyphenols, resveratrol and quercetin are poorly soluble in water. For most APIs that have poor water solubility, methods such as spray drying, coprecipitation, solvent casting/evaporation and formation of amorphous dispersions of the API in edible polymers have been employed for the formulation of dosage forms. In the past, the effectiveness of these techniques has been well established and documented in the pharmaceutical industry.

Spray drying technology:

The use of spray drying technology in the foods and pharmaceutical industry is eminent as it has often been utilized for producing dry powders from a liquid product. In the pharmaceutical industry, it is employed especially when thermally-sensitive products are involved. In this process, the API and the excipients such as a polymer, plasticizer, colorant and other common materials are thoroughly mixed in a solvent vehicle, allowed to completely dissolve and then forced through a spray nozzle or atomizer that has a pre-determined pore size. As the media passes through the nozzle, a drying gas that is heated to a certain temperature is passed through the nozzle to 
yield the final product. An important advantage that this method possesses is the control over particle size. Thus, depending on the equipment used, the drop size can be varied from 10 to 500 micrometers. Various types of nozzles are used in the process of spray drying some of which commonly include the rotary nozzle and the single-fluid pressure swirl nozzle. Hot air is widely used for drying the liquid media but in the case of an inflammable liquid, nitrogen is used. The final dry product obtained, is usually a freely flowing powder. By utilizing either co-current or counter-current flow of the hot drying gas, residence times of the media within the atomizer or spray nozzle can be varied. Hence, longer residence times are obtained with counter-current flow while shorter residence times are obtained with co-current flow of the drying gas $(33,34$, $35)$.

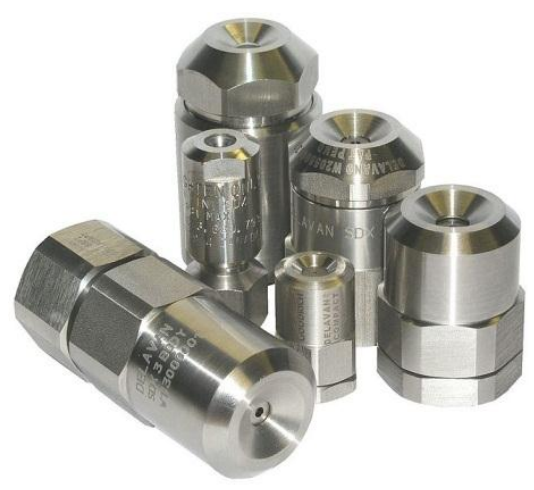

Figure 6: SDX Spray drying nozzle (36)

Solvent evaporation technique:

A number of organic solvents such as ethanol, acetone, dimethyl formamide, cyclohexane, methanol and others are widely used in pharmaceutical manufacturing. 
When poorly water soluble APIs have been involved in the formulation process, these organic solvents have been successfully employed to dissolve these poorly soluble ingredients. The method of solvent evaporation is a simple approach to formulating liquid and solid dosage forms that contain insoluble ingredients. In this technique, a solvent or a combination of miscible solvents that commonly dissolve the active compound and the other excipients required for formulation are used. These compounds are allowed to be thoroughly mixed and dissolved separately in the common solvent. The solutions obtained as a result are then mixed together and observed for signs of instability and separation. If the resultant solution remains clear and displays neither separation nor degradation, it is dried under specific conditions of heat, humidity and pressure in order to remove the solvent and obtain a uniform mixture of the final product comprising a solid solution or intimate mixture of the solvated components. An advantage of this process is its simplicity, cost reduction and shortened time of manufacturing. Major disadvantages include the use of large amounts of organic solvents that may increase the risk of toxicity and pollution and the need for energy and intensive solvent recovery (37).

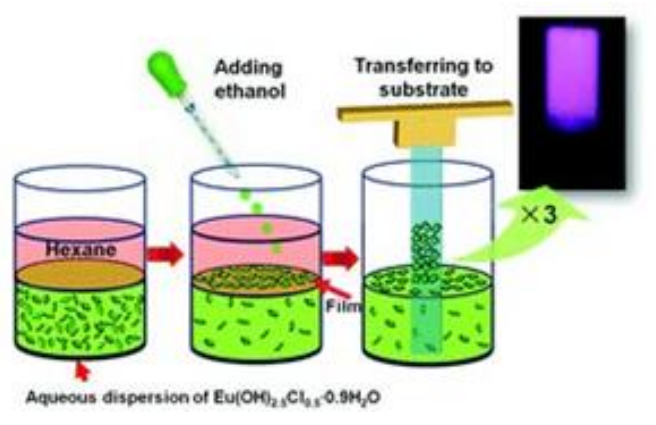

Figure 7: An example of solvent evaporation (38) 
Since, resveratrol and quercetin have very low water solubilities, these solvent-based techniques might theoretically be employed for the formulation of these polyphenols into stable dosage forms. Importantly, certain pre-formulation studies are always carried out before an API can be subjected to a particular pharmaceutical manufacturing process. With this intent, specific tests were conducted on the polyphenols to determine their characteristic properties required for their formulation using hot-melt extrusion.

The first test was conducted in order to compare the properties of the formulated products produced by hot-melt extrusion and those produced by a solvent evaporation technique. The test was designed such that two separate solutions of the polyphenol in methanol were prepared. Another solution containing a polymer and a surfactant mixed in a specific weight ratio was prepared in methanol. The two solutions were stirred until clear and then mixed together. The resultant solution was to be dried as described by the solvent evaporation technique. The product was to be formulated into tablets using the Stokes F- Press tablet punch. However, as previously mentioned, solid precipitates formed, irrespective of the order of addition or molar ratio of phenol and polymer.

Materials and methods:

For this study, Resveratrol 99\% was obtained from Changsha Organic Herb INC (Batch no. REES98-091204) and Quercetin was obtained from Acros Organics (Lot no. AO262614). The polymer selected was Kollidon VA64 (Lot no. 70-8822) and the surfactant, Lutrol F-68 (Lot no.WP1W-660E). These were obtained from BASF 
Corporation. Methanol was selected as the solvent of choice which was purchased from Fisher Scientific (Lot no.090570). In the subsequent tests, acetone was employed as the solvent, which was also obtained from Fisher Scientific (Lot no.002059).

A simple approach was first adopted to dissolve the ingredients in the solvents used. About $20 \mathrm{mg}$ of resveratrol and $20 \mathrm{mg}$ of quercetin were weighed and dispersed in two separate $100 \mathrm{ml}$ volumetric flasks containing $100 \mathrm{ml}$ of methanol. A stirring bar was added to each of the flasks and the solutions were stirred exhaustively at room temperature until clear in the absence of light. The weight ratio determined for the mixing of Lutrol F-68 and Kollidon VA64 was determined as 9:1 respectively. Thus, $90 \mathrm{mg}$ of Kollidon VA64 and $10 \mathrm{mg}$ of Lutrol F-68 were mixed physically and dispersed into a volumetric flask containing $100 \mathrm{ml}$ of methanol. A stirring bar was added to this flask and it was stirred exhaustively at room temperature in the absence of light until a clear solution was obtained.

The solutions produced in the above step were examined for presence of particles or precipitation. When none of the above was observed, the two solutions, one containing a polyphenol and the other containing the polymer and surfactant mixture were transferred into a conical flask. Upon contact of the two solutions, a thick white slurry was produced which immediately settled at the bottom of the flask while a thin layer of liquid remained as the supernatant suggesting that these materials would not be compatible in a solvent evaporation formulation process. This mixture was then dried in a vacuum oven at $30^{\circ} \mathrm{C}$ in order to dry out the solvent. When the mixture was dry, the white precipitate had changed into a hard mass that was pale brown in appearance. 
In the subsequent tests, the amount of polyphenol was varied from $2 \%$ by weight to $40 \%$ by weight of the polymer:surfactant mixture in the solvent. In another test, the solvent used was changed to ethyl acetate and acetone. In ethyl acetate, particularly heavy precipitation was observed. The addition of water to the mixture clarified the solution but did not affect the precipitation. Additionally, the order of mixing of the solutions was changed. Hence, even though the polymer-surfactant mixture was added to the API or vice versa, a similar precipitate was obtained. Depending upon the amount of polymer or the amount of API, the characteristics of the precipitate changed externally from a hard pale brown appearance to a translucent and dark brown mass. Hence, the final product obtained could not be further formulated into any specific dosage form for comparative studies with hot-melt extrusion.

Results and discussion:

The amount of polyphenol added was varied and the order of mixing of the polyphenol and the polymer-surfactant mixture was also varied. These changes in the formulation technique did not change the resultant product. The dry mass obtained on evaporation of the solvent was not suitable for formulation into tablets. Thus, solvent evaporation failed as an effective technique for the formulation of resveratrol and quercetin in Kollidon or Soluplus ${ }^{\circledR}$. It has been observed that the miscibility of Kollidon VA64 with Lutrol F-68 is low. It is hypothesized that the reason for this failure of solvent evaporation is due to this immiscibility and perhaps as a result of strong phenol-polymer interactions, a thermodynamically stable solution was not being produced on mixing the solution of the polyphenol along with a solution of Kollidon VA 64 and Lutrol F-68. Thus, it can be said that solvent evaporation is not a 
favorable technique for the formulation of the poorly soluble polyphenolic nutraceutical compounds resveratrol and quercetin.

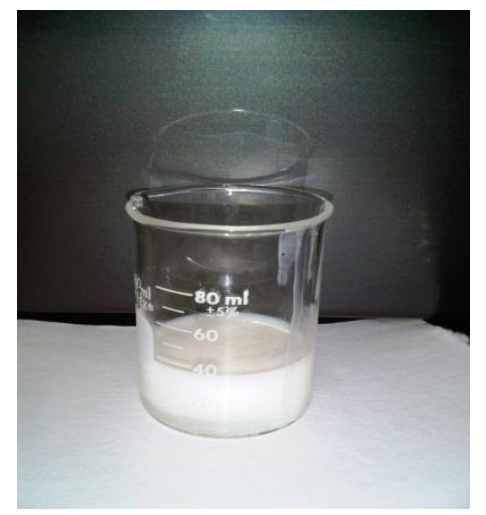

Figure 8: White precipitate obtained on mixing solutions of polymer dissolved in the solvent and resveratrol dissolved in the solvent.

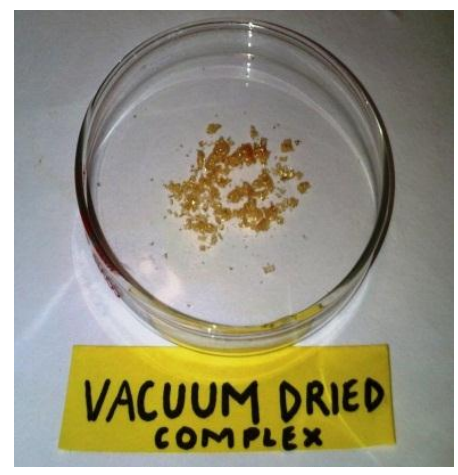

Figure 9: The white precipitate complex upon vacuum drying

\section{Conclusion:}

As observed from the above tests, the formulation of polyphenolic nutraceuticals resveratrol and quercetin could not be conducted using the solvent evaporation technique. Hence, it is hypothesized that thermodynamically stable, amorphous and dosage forms with improved solubility and bioavailability can be produced using hot- 
melt extrusion. Kollidon (and Soluplus ${ }^{\circledR}$ ) both formed precipitates with polyphenols, and were unsuitable for solvent-based formulation. Accordingly, Soluplus ${ }^{\circledR}$ was selected for further evaluation in hot-melt extrusion processing. 


\section{Chapter 4}

\section{Methodology}

\section{Study 1}

The determination of solubility and miscibility of resveratrol and quercetin in a solution of Soluplus ${ }^{\circledR}$ in water and in Phosphate buffer solution

\section{Abstract:}

The chosen polyphenols resveratrol and quercetin have very poor aqueous solubility. They have demonstrated improved solubility in organic solvents but the problem of aqueous solubility remains a concern. Soluplus® has improved the solubility of many poorly soluble compounds in water. The capacity of Soluplus® to act as a solubilizing agent in aqueous systems was examined in water. Thus, by using specific concentrations of Soluplus ${ }^{\circledR}$ in aqueous solutions and by exhaustive stirring of the polyphenols dispersed in these solutions, the improvement in solubility of the polyphenols by Soluplus® was tested. High Performance Liquid Chromatography (HPLC) analysis was used to determine the concentrations of the polyphenols in the solutions at the end of the stirring period. It was observed that Soluplus ${ }^{\circledR}$ appeared to improve the apparent solubility of resveratrol and quercetin at certain concentrations in water and in solutions of PBS. However, the improvement in the apparent solubility could not be considered to be very significant. 
Introduction

In order to produce a stable formulation that has the desirable bioavailability, the active constituents and the excipients used therein must demonstrate adequate solubility characteristics. Thus, for initiating studies on the formulation of an API, the determination of solubility in aqueous or organic solvents and the miscibility and compatibility of the selected API with the excipients such as polymers, plasticizers, solubilizing agents, preservatives and colorants becomes critical. As described earlier, Soluplus ${ }^{\circledR}$ is claimed to increase the solubility of poorly soluble drugs and has been designed specifically for hot-melt extrusion. Since it is soluble in water and various organic solvents, a test was designed for determining the effect of Soluplus ${ }^{\circledR}$ on the solubility of resveratrol and quercetin in water and other specific organic solvents. It is also essential from the point of view of melt-extrusion to determine if the selected polyphenols are miscible with Soluplus® and can be effectively subjected to the process at high temperatures and pressure to produce a stable amorphous solid solution of the polyphenols in the polymer. This test was conducted in two phases wherein water was employed as the solvent in the first phase and in the next phase phosphate buffer solution was used. Studies were designed such that they could be carried out in duplicate.

Materials and methods:

For both sets of studies, resveratrol was obtained from Changsha Organic Herb INC (Batch no. REES98-091204) and quercetin was purchased from Acros Organics (Lot no. AO262614). Soluplus® was obtained from BASF Corporation (Lot no. 
05016716KO) and de-ionized water was utilized in the first solubility and miscibility characterization study. In the second test, phosphate buffer saline (PBS) was produced by dissolution of PBS tablets produced by MP Biomedicals LLC (Lot no.2141K) in de-ionized water. Analytical studies were conducted using Hitachi High Performance Liquid Chromatography (HPLC) La-Chrom Elite. The solvent mixture used in these analytical studies consisted of 25\% methanol, $25 \%$ acetonitrile and 50\% water.

This solubility and miscibility study involved the preparation of 2 sets of 6 glass vials of $20 \mathrm{ml}$ each that contained resveratrol and quercetin dispersed separately in the concentrations of $0.625 \%, 1.25 \%, 2.5 \%$ and $5 \%$ in the solution of Soluplus $®$ in water. In addition, a saturated solution of each of the polyphenols was prepared in water and standard solutions of the polyphenols contained in methanol at a concentration of 0.5 $\mathrm{mg} / \mathrm{L}$ of methanol were prepared. These solutions were exhaustively stirred in the absence of light for 3 days. Following this, a small amount of solution from each of the samples was added to Eppendorf tubes and placed in a centrifuge that was operated for a period of 15 minutes. The tubes were then removed from the centrifuge and a small amount of the supernatant was transferred to a vial that was then analyzed by HPLC. The range of wavelength selected for the analysis was between $254-400 \mathrm{~nm}$ and the run time of HPLC analysis for each sample was selected as 20 minutes. The chromatograms obtained as a result, were assessed for their Area under the curve (AUC) values and the time of retention (tr). Using the formula,

$$
\left(\frac{\text { AUCtest }}{\text { AUCstd }}\right) \times \text { Concentration standard }
$$


the concentration of the polyphenol in each vial was calculated. Based on these results, statistical analysis in duplicate was performed on the data thus, obtained. This has been described in the subsequent chapter of results and discussion.

The determination of the solubility and miscibility of the polyphenols in PBS

Materials and Methods:

The second phase of the solubility and miscibility test was performed in order to determine the effects of micellar solubilization of Soluplus ${ }^{\circledR}$ on the polyphenols in the presence of isoosmotic salts at physiological $\mathrm{pH}$. For this test, Soluplus $®$, resveratrol and quercetin were all obtained from the sources described previously. PBS tablets were obtained from MP Biomedicals LLC (Lot no.2141K) and dispersed in de-ionized water to produce a stock solution of PBS. Each tablet made $100 \mathrm{ml}$ of PBS solution. The solvent mixture utilized previously was again employed for analytical studies by HPLC and standard solutions of the polyphenol in methanol prepared earlier were again used.

Soluplus ${ }^{\circledR}$ solutions were prepared in concentrations of $2 \mathrm{mg} / \mathrm{L}$ of PBS (below the critical micelle concentration of $7.6 \mathrm{mg} / \mathrm{L}$ ) and $16 \mathrm{mg} / \mathrm{L}$ (above the critical micelle concentration of $7.6 \mathrm{mg} / \mathrm{L}$ ) of PBS in order to evaluate micellar solubilization. Additionally, a saturated solution of Soluplus ${ }^{\circledR}$ at a concentration of $10 \%$ or $10 \mathrm{gm}$ in $100 \mathrm{ml}$ of PBS was prepared. Resveratrol and quercetin were dispersed separately in the 3 solutions of Soluplus®. A solution of the polyphenols dispersed in water was also prepared. Thus, 5 sets of solutions of each polyphenol, inclusive of the standard solutions were prepared in all and then stirred exhaustively for a period of 3 days in 
the absence of light. Following this, a small amount of the solutions were transferred into separate Eppendorf tubes and placed in a centrifuge operated for 15 minutes. The solutions were then removed and transferred into glass vials for analysis by HPLC. The solvent mixture and other conditions used previously were maintained for this experiment. As described above, the AUC values were assessed for each solution and employing the same formula, the concentration of polyphenol in each solution was calculated. Based on these results, statistical analysis in duplicate was performed on the data obtained (39).

Study 2

The formulation of polyphenolic compounds into thin films for oral delivery

\section{Abstract}

Soluplus ${ }^{\circledR}$ is a polymeric solubilizer and has demonstrated satisfactory film forming ability with a few active compounds. These poorly soluble compounds can be formulated into films for topical or oral delivery by utilizing a solution of Soluplus ${ }^{\circledR}$ dissolved in a specific solvent in a certain concentration. Resveratrol and quercetin were tested in such film formulations by using dimethyl formamide (DMF) as the solvent. A solution of Soluplus ${ }^{\circledR}$ was prepared in DMF in a specific concentration of $2 \mathrm{~g} / \mathrm{L}$ and the polyphenols were added separately to this solution and stirred exhaustively. They were laid out as films on a clean glass plate and dried. The films were observed upon drying and revealed that the polyphenols remained undissolved and showed traces of re-crystallization. Further, undesirable results such as cracking and breaking of the films were observed. 
Introduction

Films for topical delivery have been extensively formulated for numerous active compounds. They serve as an effective method of drug delivery when oral formulations cannot be employed. Compounds that have been poorly water soluble were successfully dispersed in creams, lotions, suspensions, ointments and transdermal patches and delivered for adequate pharmacological effects. This route of administration possesses the added advantage of escaping the first pass hepatic and GIT metabolism which is often observed with orally delivered dosage forms. Another advantage it possesses is direct delivery to the site of action, especially when the skin or the oral mucosa is the target tissue. As mentioned earlier, topical creams and films of resveratrol in order to take advantage of its anti-aging effects have been produced. Soluplus ${ }^{\circledR}$ is claimed to be an effective tool in film formulation and improves the bioavailability of compounds incorporated with Soluplus ${ }^{\circledR}$ as a film. Accordingly, a study was designed to formulate films of resveratrol and quercetin in Soluplus $®$ and test their characteristics.

Materials and Methods:

For this study, resveratrol was obtained from Changsha Organic Herb INC (Batch no. REES98-091204), quercetin was obtained from Acros Organics (Lot no. AO262614) and Soluplus@ was purchased from BASF Corporation (Lot no.05016716KO). The solvent selected for this study was dimethyl formamide (DMF). This solvent was obtained from Fisher Scientific (Lot no.893941). A vacuum oven was used for drying 
the films. A compound microscope was used for observing the films for traces of crystals.

The study was designed such that increasing concentrations of the polyphenol could be incorporated into the solution of Soluplus ${ }^{\circledR}$ in DMF that would eventually be dried to obtain the films. Concentrations of resveratrol and quercetin were fixed at $20 \%$, $30 \%$ and $50 \%$ of the polymer solution by weight. Thus, 2 grams of Soluplus ${ }^{\circledR}$ were dispersed in $100 \mathrm{ml}$ of DMF. The solution was exhaustively stirred at room temperature. The required quantities of resveratrol and quercetin were then dispersed separately in the solution of Soluplus® in DMF. Hence, 3 solutions of each polyphenol prepared were stirred for 3 days in the absence of light. The solutions were then carefully laid out as thin films on a flat surface and placed in a vacuum oven maintained at $20^{\circ} \mathrm{C}$ for 4 hours. Additionally, a blank film of the polymer was prepared from Soluplus ${ }^{\circledR}$ for comparison and dried in the same manner as the polyphenol films. The films were then observed both visually and under the microscope for the presence of cracks and crystals or more elaborately, signs of recrystallization and instability and have been described in the next chapter (39).

Study 3

The assay of content and determination of its uniformity of the hot-melt extruded mixtures of quercetin and resveratrol.

\section{Abstract}

The content uniformity of a pharmaceutical product is critical from the point of view of pharmaceutical processing and formulations. Changes in the content of the active 
constituents are indicative of the undesired effects produced on the product during processing, packaging and storage. The determination of the content uniformity can be carried out in numerous ways and one common method utilized is analytical study by separation or chromatography. In this study, weight normalized samples of the HME mixtures of resveratrol and quercetin in Soluplus ${ }^{\circledR}$ were allowed to be stirred exhaustively in a combination of methanol and water. These samples were analyzed by high performance liquid chromatography and the chromatograms obtained as a result were observed for their retention times and AUC values which correlate with phenolic content and stability. A superimposition of the chromatograms, study of the area under the curve values and observation of retention times provided information on the content uniformity. The data indicated that different samples of resveratrol and quercetin in Soluplus ${ }^{\circledR}$, both physically mixed and hot-melt extruded, had similar retention times and superimposable peaks indicating content uniformity.

Introduction

In the pharmaceutical industry, raw materials required in the production of dosage forms are tested for their quality and purity before beginning the manufacturing process. It is important that the materials remain stable and maintain their characteristics throughout the production process, and more so, during storage. These tests assure that the materials are of high quality and functionality and are effective in their viability in a particular product. Thus, with a similar intent, it is important to test the uniformity of the hot-melt extruded mixtures of the selected polyphenols and Soluplus ${ }^{\circledR}$ in order to determine their purity and quality. The following content 
uniformity assay was thus carried out using HPLC for the above mentioned purpose (40).

Materials and Methods:

Resveratrol and quercetin that were hot-melt extruded were obtained from Foster Corporation. Soluplus® was obtained from BASF and resveratrol produced by Changsha Organic Herb INC (Batch no. REES98-091204) and quercetin obtained from Acros Organics (Lot no. AO262614) was used. A mixture of methanol and water was used as a solvent in the ratio of 1:1. Methanol (Lot No-060074) was obtained from Fisher Scientific. The solvent mixture used for the analytical studies in the case of the resveratrol solutions consisted of $25 \%$ methanol, $25 \%$ acetonitrile and $50 \%$ water. The analysis was performed on the Hitachi La-Chrom Elite using the ODS -2 Hypersil C18 column (dimensions in mm-150x4.6; particle size-5 $\mu$ ) produced by the Thermo Electron Corporation.

Methanol and de-ionized water were mixed in the ratio of 1:1. Thus, a $200 \mathrm{ml}$ stock solution containing $100 \mathrm{ml}$ methanol and $100 \mathrm{ml}$ water was prepared at the start. Resveratrol and quercetin were then dispersed in the solution prepared above such that concentrations of $0.0625 \mathrm{mg} / \mathrm{ml}, 0.1 \mathrm{mg} / \mathrm{ml}, 0.125 \mathrm{mg} / \mathrm{ml}, 2.5 \mathrm{mg} / \mathrm{ml}$ and $0.5 \mathrm{mg} / \mathrm{ml}$ by weight were produced for a thorough comparison. In addition, weighed samples of 5\% and $20 \%$ of the HME mixtures of resveratrol or quercetin were added to the methanolwater solution. Hence, seven sets of solutions for each polyphenol were produced. Lastly, a blank methanol, blank water and blank methanol- water solution were prepared for comparison. These solutions were stirred continuously in the absence of 
light for 3 days at room temperature. A small amount was then drawn from each solution and placed in glass vials for analysis by HPLC. The chromatograms obtained as a result were assessed for the AUC values to determine the uniformity of content. These have been described in more detail in the next chapter.

Study 4

A study of the dissolution characteristics of resveratrol in gelatin capsules

\section{Abstract}

The study of dissolution is important from the point of view of predicting the release of a drug from a pharmaceutical dosage form within the body. This in-vitro study is often carried out on oral dosage forms such as tablets and capsules in order to evaluate their solubility and bioavailability properties. Resveratrol present in a concentration of $5 \%$ by weight in a mixture either melt extruded with Soluplus ${ }^{\circledR}$ and physically mixed with Soluplus® was chosen for the study. Thus, the solubilizing effect of melt extrusion into Soluplus® versus the solubilizing effects of Soluplus® alone could be evaluated. In order to study the effect of particle size on dissolution, two size fractions of the HME mixture were prepared with particles having a size of $250 \mu \mathrm{m}$ and $425 \mu \mathrm{m}$. These were filled into hard gelatin capsules. Correspondingly, capsules containing the physical mixture of resveratrol and Soluplus ${ }^{\circledR}$ in the same ratio be weight were prepared. Dissolution studies were conducted in simulated intestinal fluid and the results were analyzed by HPLC. The results showed the effect of the smaller particle size on dissolution. The release profile of the capsules containing the physical mixture and the HME mixture with a particle size of $180-250 \mu \mathrm{m}$ had a more rapid release rate 
and higher release than the capsules containing the HME mixture with a particle size of $250-425 \mu \mathrm{m}$.

Introduction

Dissolution is the process wherein the active constituent from a dosage form is released into the gastrointestinal fluid within the body. This compound, once released, will then ideally be absorbed into the systemic circulation which will carry the compound to its site of action. Dissolution studies are conducted in order to mimic the gastric and intestinal conditions and predict the rate and extent of drug released into the system. It also assists in predicting the effectiveness of a particular dosage form in drug delivery and the rate at which the drug is released from this product. Some products are designed to be released very quickly, while some are designed for slow and steady release properties. Thus, depending on whether absorption occurs in the stomach or in the intestine, the medium used in the dissolution study can be varied from a simulated gastric fluid to a simulated intestinal fluid. This in-vitro study also takes into consideration the body temperature and thus, the medium can be maintained at $37^{\circ} \mathrm{C}$ for the length of the study. The following study was designed with the intention of comparing the release profile of resveratrol that was physically mixed with Soluplus ${ }^{\circledR}$ to that of resveratrol that was melt extruded with Soluplus ${ }^{\circledR}$. Hence, the aim of this experiment was to test the improvement in solubility, and appreciate the potential bioavailability, of resveratrol after extrusion with Soluplus ${ }^{\circledR}$ as hypothesized earlier. The HME resveratrol and the resveratrol that was physically mixed with Soluplus ${ }^{\circledR}$ were filled into hard gelatin capsules of size 1 , the capsules 
placed in sinkers, and dissolution was assessed in a Type II basket apparatus, USP (41).

Materials and Methods:

Resveratrol 5\% HME was selected for this study. Resveratrol obtained from Changsha Organic Herb INC (Batch no. REES98-091204) and Soluplus® obtained from BASF Corporation (Lot no.05016716KO) were used in the preparation of the physical mixture. Capsules of size 1, obtained from Capsugel, were used for the test. The dissolution medium employed for the study was simulated intestinal fluid or a solution with a $\mathrm{pH}$ of 6.8 . This was prepared using monobasic potassium phosphate, sodium hydroxide and de-ionized water and was adjusted to a $\mathrm{pH}$ of $6.8+0.1$ with sodium hydroxide $1 \mathrm{~N}$. The dissolution apparatus used for the study was the Vanderkamp® 600 Dissolution tester. Sieves of $180 \mu \mathrm{m}, 250 \mu \mathrm{m}$ and $425 \mu \mathrm{m}$ were employed for sieve distribution of particles based on size. A twin blade rotary mill was used for the reduction of particle size. A Stokes F-Press tablet punching machine; model 519-2, was employed for pressing the mixtures.

The batch size of the physical mixture of resveratrol and Soluplus ${ }^{\circledR}$ was fixed as 100 grams. About $5 \mathrm{gm}$ of resveratrol was weighed and mixed with $95 \mathrm{gm}$ of Soluplus ${ }^{\circledR}$. This mixture was then placed in a turbula blender for 20 minutes to allow fine blending of the powders. About 20 grams of the 5\% HME mixture was ground in a twin blade rotary mill for about 10 seconds. This mixture was then pressed under the Stokes F-Press tablet machine. This was carried out in order to mimic the conditions of a roller compactor in the industry. The mixture was then passed through a set of 
sieves placed in order of descending sieve size and distributed and collected according to the sieve size. Thus, two sets of the HME mixtures were prepared, one having a particle size of $250 \mu \mathrm{m}$; and the other a size of $425 \mu \mathrm{m}$. Hence, overall, three mixtures were prepared for the dissolution study. These included the physical mixture of resveratrol and Soluplus ${ }^{\circledR}$, the HME mixture of resveratrol and Soluplus ${ }^{\circledR}$ with a size fraction of $180-250 \mu \mathrm{m}$ and the HME mixture of resveratrol and Soluplus ${ }^{\circledR}$ with a size fraction of $250-425 \mu \mathrm{m}$. The simulated intestinal fluid (SIF) was prepared by dissolving 6.8 grams of monobasic potassium phosphate in $250 \mathrm{ml}$ of water and adding $77 \mathrm{ml}$ of $0.2 \mathrm{~N}$ sodium hydroxide dissolved in $500 \mathrm{ml}$ of water. The volume of SIF was then increased in order to suit the experimental needs. The $\mathrm{pH}$ was further adjusted with $0.2 \mathrm{~N}$ sodium hydroxide to 6.8 . This was used as the dissolution medium and added to the dissolution vessels in the apparatus which were filled with the SIF up to a volume of $600 \mathrm{~mL}$. The temperature of the medium was maintained at $37^{\circ} \mathrm{C}$ using a water bath. The mixtures prepared were filled into hard gelatin capsules of size 1 such that 3 capsules were prepared for each type of mixture. Thus, 9 capsules were prepared in all for the study. A capsule sinker was used in order to prevent them from floating to the surface of the medium in the vessels and causing an error in measurement. The spindles were lowered into the vessels and the spindles were operated at a speed of $75 \mathrm{rpm}$. The initial sample was taken out at time 0 immediately after the capsules were then dropped into the vessels. Subsequent samples were then withdrawn at 15 minutes, 30 minutes, 45 minutes, 60 minutes and 90 minutes after the first measurement. These samples were placed into glass vials and were then analyzed by HPLC. For the analytical assay, a mixture of $25 \%$ methanol, $25 \%$ acetonitrile and 
$50 \%$ water by volume was used as the mobile phase with using an ODS -2 Hypersil C18 column. The chromatograms obtained were assessed for their AUC values and based on this data; the concentration of substance released at the specific time points was calculated. This data was further plotted against the time intervals to determine the release characteristics of the capsules which have been described in the subsequent chapter.

\section{Study 5}

The characterization of molecular interactions between resveratrol and Soluplus and quercetin and Soluplus on hot-melt extrusion using Fourier transform infra-red spectroscopy

\section{Abstract}

FT-IR spectroscopy has been widely employed for determining the chemical nature of a compound and is an important tool in identifying and characterizing compounds. This technique uses infra-red radiation to assist in identifying and determining certain characteristic types of interactions that occur within a molecule of the compound. Chemically, compounds may contain aromatic moieties, carbonyl carbons, alcohol groups, amide bonds and other types of functional groups. These groups undergo bending and stretching depending upon their chemical bonding due to the infra-red radiation they are exposed to in FT-IR spectroscopy. The instrument records these interactions and assists in the identification of the compound. Resveratrol and quercetin are polyphenols and Soluplus ${ }^{\circledR}$ is a graft co-polymer. These compounds when formulated in hot-melt extrusion interact intimately with each other. The 
following study was carried out in order to analyze these interactions and determine the characteristics of the HME mixtures. The results showed that the chemical properties of the polyphenols undergo a change after extrusion with the polymer. The properties of the HME mixtures of resveratrol and quercetin closely resemble the properties of Soluplus® after extrusion.

\section{Introduction}

The technique of Fourier transform infra-red spectroscopy (FT-IR) has been utilized for studying and predicting molecular interactions within a given compound. This technique is based on the measurement of infra-red radiation absorbed or transmitted by a compound. The constituent molecules within the compound have characteristic vibrations about their bonds when subjected to infra-red radiation. These molecules absorb some of this radiation and emit the rest. This emitted light is detected by a specific detector built into the instrument which converts the wavelengths based on a mathematical algorithm called a Fourier transform. Thus, a spectrogram is obtained for a compound which displays the peaks of absorbed or transmitted wavelengths which are characteristic of certain chemical groups present within a molecule. The following study was used to detect and analyze the interactions that may have occurred between the molecules of resveratrol and Soluplus ${ }^{\circledR}$ and quercetin and Soluplus ${ }^{\circledR}$ on being melted and extruded at high temperatures (42).

\section{Materials and Methods}

Resveratrol and quercetin HME mixtures were obtained from Foster Corporation. They were prepared in the concentrations of $2 \%, 5 \%$ and $20 \%$ by weight for 
resveratrol and $5 \%$ and $20 \%$ by weight for quercetin. The pure samples and the physical mixtures were prepared using Resveratrol $99 \%$ obtained from Changsha Organic Herb INC (Batch no. REES98-091204) and quercetin obtained from Acros Organics (Lot no. AO262614) in Soluplus® (Lot no.05016716KO) obtained from BASF Corporation. Clean glass vials were used for storing the samples and spectroscopy was performed on the Nicolet 380 FT-IR by Thermo Electron Corporation.

A small amount, about $5 \mathrm{mg}$ of each of the HME mixtures of resveratrol and quercetin were placed in clean glass vials. For a comparative study, physical mixtures of the polyphenols in Soluplus ${ }^{\circledR}$ were prepared in concentrations of $2 \%, 5 \%$ and $20 \%$ by weight of resveratrol and $5 \%$ and $20 \%$ by weight of quercetin. These mixtures were blended by gentle shaking and then placed in a turbula blender for efficient mixing. The mixtures were then filled into clean glass vials and used for analytical spectroscopy. The instrument required no sample preparation and thus, the samples were directly placed in the ZnSe (Zinc Selenium) cell for analysis. Each sample was analyzed for a period of three minutes using thirty seven scans. The spectrograms obtained were labeled with the required data and a comparative study was carried out. The results and the interactions observed have been described further in the next chapter. 
Study 6

A study of the stability, thermal properties and accelerated thermal degradation of resveratrol and quercetin using differential scanning calorimetry (DSC)

\section{Abstract:}

The technique of DSC is used for studying the behavior of a substance under conditions of varying temperature. This technique is helpful in determining the melting point of a given compound and provides information on the chemical constitution of a compound. It is often used for analyzing polymeric compounds and helps in understanding various inherent characteristics of polymers such as crystallization and glass transition temperatures. Importantly, DSC can be used for studying pharmaceutically formulated products that may contain insoluble or poorly soluble constituents for separation or recrystallization which is undesired and often is a sign of instability. In this study, the HME mixtures of resveratrol and Soluplus $®$ and quercetin and Soluplus ${ }^{\circledR}$ were assessed for their stability using DSC. In a separate study, the physical mixtures of resveratrol and Soluplus ${ }^{\circledR}$ and quercetin and Soluplus ${ }^{\circledR}$ were analyzed using the DSC in order to determine the behavior of these mixtures under heating and immediate cooling. By carrying out this study, information on the behavior of the polyphenol-polymer mixtures in the process of extrusion could be determined. The DSC results showed that quercetin acted as a plasticizer for Soluplus ${ }^{\circledR}$ and produced a depression in the glass transition temperatures at a higher concentration. Additionally, in the HME mixtures, the melting point of the resveratrolSoluplus ${ }^{\circledR}$ mixtures was significantly higher than the melting point of pure resveratrol 
while the melting point of the quercetin-Soluplus ${ }^{\circledR}$ mixture was reduced as compared to pure quercetin when higher concentrations of quercetin were present in the mixture.

Introduction:

The technique of differential scanning calorimetry measures the difference in the amount of heat required to increase the temperature of a sample as compared to the temperature of a reference or blank sample. This thermoanalytical technique may be useful for determining the behavior of a substance under conditions of increasing heat or under rapid changes in temperature. Throughout the test, the sample and the reference are maintained at nearly the same temperature. Any change in the amount of heat required for the sample to remain isothermic is often displayed as a peak or a sharp trough. The instrument used for this type of analysis is called the differential scanning calorimeter (DSC) and the results it displays are referred to as thermograms. Most importantly, these thermograms assist in the detection of the melting point of a compound and also help predict its behavior at elevated temperatures. The glass transition temperature or the temperature at which a compound changes from the glassy to the liquid crystal state is also often analyzed by the DSC. By studying the DSC thermogram, it is possible to predict whether a compound or more specifically, a formulation, would undergo a transition from an amorphous to the crystalline state under conditions of storage. Re-crystallization of the poorly soluble or insoluble ingredients in a formulation is an area of concern. This instability can be effectively detected by means of DSC. The following study was designed in order to better understand the behavior of resveratrol and quercetin at high temperatures as the process of hot-melt extrusion involves melting and fusion under pressure and heat. In 
addition, the stability of the melt extruded mixtures was assessed using DSC to detect traces of crystallinity or instability $(29,43,44,45)$.

Materials and methods:

Resveratrol 99\% was obtained from Changsha Organic Herb INC (Batch no. REES98091204) and quercetin from Acros Organics (Lot no. AO262614). Soluplus ${ }^{\circledR}$ (Lot no.05016716KO) was obtained from BASF Corporation. The HME mixtures of resveratrol and quercetin in Soluplus ${ }^{\circledR}$, prepared in the concentrations of $2 \%, 5 \%$ and $20 \%$ by weight of resveratrol and $5 \%$ and $20 \%$ by weight of quercetin were obtained from Foster Corporation. The instrument used for the thermoanalytical study was the Q10 Differential Scanning Calorimeter produced by TA instruments. Clean glass vials were used for storing the samples. The pans used for the tests were obtained from TA instruments and were the Tzero Hermetic pans.

Resveratrol, quercetin and Soluplus ${ }^{\circledR}$ were filled into separate clean glass vials. The HME mixtures of resveratrol and quercetin were filled into their respective vials. For a comparative study, small amounts of the physical mixtures of the polyphenols in Soluplus ${ }^{\circledR}$ were prepared and blended using the turbula blender. The weight fractions of the polyphenols were maintained analogus to the HME mixtures. Additional physical mixtures of resveratrol and quercetin in Soluplus ${ }^{\circledR}$ were prepared with increasing concentrations of the polyphenol in Soluplus ${ }^{\circledR}$. Thus, physical mixtures of $30 \%$ and $50 \%$ by weight of the polyphenols were prepared in Soluplus ${ }^{\circledR}$. These samples were filled into the hermetic pans and crimped as prescribed. The melting point of resveratrol is $254^{\circ} \mathrm{C}$ and that of quercetin is $316^{\circ} \mathrm{C}$. The method for 
resveratrol and quercetin was set such that the run involved heating to $280^{\circ} \mathrm{C}$ for resveratrol and $380^{\circ} \mathrm{C}$ for quercetin when the physical mixtures were tested. The hotmelt extruded mixtures were tested at temperatures below the melting point of the polyphenols to mimic the temperature conditions of extrusion. In a separate test, the HME mixtures were subjected to heating at a rate of $10^{\circ} \mathrm{C} / \mathrm{min}$ from an initial temperature of $40^{\circ} \mathrm{C}$ to a temperature of $380^{\circ} \mathrm{C}$ for resveratrol and $450^{\circ} \mathrm{C}$ for quercetin. The samples were then cooled to the initial temperature and then tested again at the same temperature range. The endotherms obtained as a result, were studied for the characteristics exhibited by these samples. These thermograms and the results have been described in the next chapter.

Study 7

A study of the properties of tablets produced from resveratrol and quercetin

\section{Abstract:}

Tablets are among the most commonly administered oral dosage forms. Tablets are produced in a variety of sizes, shapes and colors and are used for delivery into the GIT, buccal delivery and sublingual delivery. When tablets are manufactured in the pharmaceutical industry, they are tested for a number of characteristic properties which are important in determining the quality and standard of the tablet produced. These include tests to determine the hardness of the tablet, tests on friability and disintegration time of the tablet among others. In this study, the formulation of resveratrol and quercetin into tablets was carried out and the properties of the tablets were studied using the characterization tests such as the hardness test, friability test 
and the disintegration test. The process involved direct compression of the HME mixtures of resveratrol and Soluplus ${ }^{\circledR}$ and quercetin and Soluplus ${ }^{\circledR}$ in a Stokes FPress tablet machine to produce tablets of these mixtures and the compression of the physical mixtures of resveratrol and Soluplus ${ }^{\circledR}$ and quercetin and Soluplus ${ }^{\circledR}$ using the technique and machine into tablets. These tablets were then analyzed using the tests mentioned above. The tests indicated that the tablets produced from the HME mixtures of quercetin and Soluplus® were harder and less friable than the tablets produced from the HME mixtures of resveratrol and Soluplus®. The disintegration times of the tablets produced from the HME mixtures and the physical mixtures were variable.

Introduction:

Tablets are the most widely produced dosage forms for oral drug delivery. They include the active compound and other ingredients referred to as excipients, which serve as diluents, glidants and are often necessary for the development of a tablet from its crude powder form. The excipients mainly include binders that hold the powder and prevent it from breaking, diluents, colorants, flavorants, disintegrating agents and lubricants. These materials are required at various steps of the manufacturing process for tablets. Tablets can also be produced to deliver the drug sublingually or through the buccal cavity. The ingredients and manufacturing processes can be varied to produce slow -releasing or sustained release tablets. Polymers such as polyvinyl pyrrolidone (PVP) have often been used as binders in tablets as well as cellulose, starch and gum acacia. Tablets can be produced in various shapes depending upon the type of machine that is used for manufacturing. These machines consist of a die and punches wherein the crude mixture of powders to be pressed into a tablet (present in 
the form of granules) is placed in the die and the combination of upper and lower punches compresses the mixture into a tablet of the desired shape. Thus, by using different combinations of punches and dies and varying other parameters such as pressure exerted on the granules and fill capacity, the size and shape of the tablet can be changed.

A tablet produced in this manner must possess sufficient hardness in order to sustain the rigors of the packaging, transport and storage processes. The tablet must not show signs of cracking, chipping, discoloration and capping which are considered as signs of brittle or unstable tablets. There are standard tests conducted on tablets to detect such problems and others which may show inadequacies in the formulation process. A hardness test is conducted to check if the tablet is hard enough to remain stable during its shelf-life and at the same time has sufficient breaking/disintegrating characteristics. A friability test is conducted to determine whether the tablet has been formulated such that it does not discharge the constituents in the form of dust or break easily during its shelf-life $(46,47,48)$.

Disintegration is the first step before dissolution of a tablet delivered orally occurs. In this process, a tablet absorbs the fluid in which it is present such as the gastric or intestinal fluid and swells to break into small fragments. These fragments undergo dissolution and release the drug which is then absorbed into the systemic circulation. Thus, an in-vitro disintegration test is performed to observe the time required for the tablet to swell and break into smaller fragments. This is carried out in simulated gastric fluid or simulated intestinal fluid at a temperature of $37^{\circ} \mathrm{C}$. The following study was designed in order to test the characteristics of the tablets produced by 
resveratrol and quercetin using the HME mixtures and the corresponding physical mixtures of these polyphenols in Soluplus ${ }^{\circledR}$. The tablets produced were tested for their physical appearance, hardness, and friability and disintegration properties (49).

Materials and methods:

Resveratrol 99\% was obtained from Changsha Organic Herb INC (Batch no. REES98091204) and quercetin procured from Acros Organics (Lot no. AO262614) were utilized and Soluplus® obtained from BASF Corporation (Lot no.05016716KO). The HME mixtures were prepared by Foster Corporation. Resveratrol and Soluplus® mixtures were prepared in concentrations of $2 \%, 5 \%$ and $20 \%$ by weight of resveratrol and quercetin in Soluplus ${ }^{\circledR}$ was prepared in concentrations of $5 \%$ and $20 \%$ by weight of quercetin. The corresponding physical mixtures were prepared for resveratrol and quercetin in Soluplus®. Magnesium stearate obtained from Sigma Chemical Company Lot no 110-F0064 was used as a lubricant in the powder and HME mixtures. The Stokes F-Press Model 519-2 tablet machine was used for the production of the tablets. Disintegration was performed on the LIJ-3 Disintegration tester by Vanguard Pharmaceutical Machinery Inc. and the hardness was tested using an Erweka tablet hardness tester. The Erweka friabilator was used for performing the friability test on the tablets produced.

The HME mixtures of resveratrol and quercetin were first selected for the test. About 10 grams of each mixture was first ground and sieved to obtain uniformity of particle size. It was claimed that hot-melt extrusion of the polyphenols with Soluplus ${ }^{\circledR}$ produces directly compressible tablets. Hence, the need of a binder was eliminated. 
The mixtures produced by grinding and sieving were then placed in separate containers to be passed through the Stokes F-press for the production of tablets. The machine parameters were adjusted such that the pressure was maintained close to maximum and the fill capacity was adjusted to yield tablets of $200 \mathrm{mg}$ each. Initial tests were performed with a test mixture to check the weight of the tablets produced. When satisfactory results were obtained, the mixtures were compressed using the machine. The total weight of the all the tablets produced for each mixture was measured along with the individual weights of the tablets to prevent errors in the tests performed.

The physical mixtures of resveratrol and quercetin with Soluplus ${ }^{\circledR}$ were prepared in the same weight fractions as that of their corresponding HME mixtures. About 10 grams of each physical mixture was prepared by blending in a turbula blender for efficient mixing. The mixtures were ground and sieved for maintaining uniformity in particle size. Magnesium stearate was added to the mixtures and they were then filled in separate containers for compression by the F-Press. The pressure and the fill capacity were retained similar to the compression of the HME mixtures. The tablets prepared were then weighed using an electronic balance (Denver Instruments weighing balance; Model no-XL-410D) in a manner similar to the tablets of the HME mixtures.

The first test performed on the tablets was the hardness test. Five tablets were chosen for each of the mixtures and placed in the tester. The results were recorded as kilograms of weight required to break the tablet. 
The friability test was then performed on the tablets. Ten tablets of each type were selected for this test. Their individual weights were measured and totaled to give the average weight of the tablet. They were then placed in the Erweka Friabilator for 20 minutes at a speed of $15 \mathrm{rpm}$. At the end of this period, the friabilator was stopped and the tablets were removed and weighed. The difference in weight was calculated and the percent friability recorded. Thus, the test was performed with a batch of 10 tablets of each mixture placed in successive runs.

The final test performed on the tablets was disintegration. The instrument selected for this purpose was the LIJ-3 disintegration tester. The instrument was maintained at $37^{\circ} \mathrm{C}$ prior to the test. For this test, 3 tablets from each mixture were selected and weighed. These were then placed in a vessel containing simulated intestinal fluid $(\mathrm{pH}$ $6.8 \pm 0.1)$. The instrument controls were set to a period of 15 minutes and the test was then initiated. The time required for the tablets to swell, crack and then break was noted. The data obtained for the HME mixtures was then compared with the data obtained for the corresponding physical mixture.

\section{Study 8}

A study of the change in the properties of the hot-melt extruded mixtures under forced degradation at conditions of elevated temperature and humidity

\section{Abstract:}

The process of forced degradation involves the hastened degradation of the pharmaceutical product or dosage form in order to study the effects of varying environmental conditions on the overall stability and quality of the pharmaceutical 
product. In this process, the product is commonly placed in an environment with an elevated temperature and controlled humidity conditions. Most often, chemical reactions that may produce degradation within the pharmaceutical product are accelerated or triggered in the presence of heat and humidity and degradation occurs at a faster rate than under normal conditions of temperature (storage conditions of $25^{\circ} \mathrm{C}$ ). The HME mixtures of resveratrol and Soluplus ${ }^{\circledR}$ and quercetin and Soluplus ${ }^{\circledR}$ were placed in an oven maintained at $40^{\circ} \mathrm{C}$ and $75 \%$ relative humidity for one month. At the end of this period, the mixtures were stirred exhaustively in methanol and analyzed by HPLC. From the chromatograms obtained and on comparison of these chromatograms with the chromatograms of the mixtures obtained prior to degradation it was observed that the mixtures may perhaps undergo degradation under these conditions wherein there may be a significant decrease in the concentration of the active polyphenol within the HME mixture.

Introduction:

Forced degradation is a process of hastening the degradation of a substance or dosage form under conditions of stress. Different types of stress conditions can be applied for this purpose which includes heat, humidity, and changes in $\mathrm{pH}$, oxidation, concentration and light amongst others. This type of study is commonly carried out on pharmaceutical products in order to determine whether the finished products maintain their characteristic properties of stability under various conditions. The study also assists in analyzing the ideal conditions required for storage of the product in order to prevent its degradation to an unstable, hazardous or toxic and inactive form. In case of hot-melt extrusion, the common signs of instability are re-crystallization of the active 
compound in the product and separation or discoloration. The goal of hot-melt extrusion technology is to produce a stable amorphous solid solution of the drug in the polymer. Hence, it is essential to study these extruded mixtures for their stability under various conditions. With this intent, the flowing study was designed to determine the change in uniformity of content of the extruded products after storage at an elevated temperature of $40^{\circ} \mathrm{C}$ and relative humidity controlled at $80^{\circ} \mathrm{C}(50)$.

Materials and methods:

Hot-melt extruded mixtures of Resveratrol in Soluplus ${ }^{\circledR}$ prepared in concentrations of $2 \%, 5 \%$ and $20 \%$ by weight of resveratrol were obtained from Foster Corporation. Similarly, HME mixtures of quercetin in Soluplus ${ }^{\circledR}$ prepared in concentrations of $5 \%$ and $20 \%$ by weight of quercetin were obtained from Foster Corporation. A mixture of $25 \%$ methanol, $25 \%$ acetonitrile and $50 \%$ water was used as the mobile phase along with a C18 column for separation by HPLC.

About 10 milligrams of each mixture was filled into separate clean glass vials and placed in an oven, Stabil-Therm ${ }^{\circledR}$ Blue M, with a temperature controlled at $40^{\circ} \mathrm{C}$ and relative humidity at $80 \%$ for a period of one month. At the end of this time period, the vials were removed from the oven and allowed to be exhaustively stirred in methanol for 3 days in the absence of light. Small amounts of the resultant solutions were drawn into clean glass vials to be analyzed by HPLC. The chromatograms obtained as a result were compared to the chromatograms obtained for the mixtures before the forced degradation was initiated. These results have further been described in the following chapter. 


\section{Chapter 5}

\section{Results and findings}

\section{Study 1}

The determination of solubility and miscibility of resveratrol and quercetin in a solution of Soluplus ${ }^{\circledR}$ in water and in Phosphate buffer solution.

Results:

The study was designed in order to determine the effect of Soluplus ${ }^{\circledR}$ on the solubility of the poorly soluble polyphenolic compounds in water and additionally, test the effects of the micellar solubility of Soluplus ${ }^{\circledR}$ on these polyphenols in phosphate buffer solution. The samples were prepared as described in the methodology chapter for the first part of this study, namely, solubility determination in water were analyzed by HPLC.

The chromatograms obtained were assessed for the AUC values and the concentrations were calculated from the formula described earlier. These values were tabulated as shown further. 


\begin{tabular}{|l|l|l|l|l|l|}
\hline Compound & \multicolumn{4}{l}{ Solution } & \multicolumn{4}{l|}{} \\
\cline { 3 - 6 } & \multirow{2}{*}{ Water } & \multicolumn{4}{l|}{ Soluplus ${ }^{\circledR} \% \mathrm{w} / \mathrm{w}$} \\
\cline { 3 - 6 } & & $0.625 \%$ & $1.25 \%$ & $2.5 \%$ & $5 \%$ \\
\hline Resveratrol & 0.038 & 0.224 & 0.364 & 0.211 & 0.011 \\
\hline Quercetin & 0.634 & 1.589 & 2.178 & 2.42 & 1.46 \\
\hline
\end{tabular}

Table 4: Data of the concentration of the apparent solubilities of resveratrol and quercetin in solutions of serial concentrations of Soluplus ${ }^{\circledR}$ in water

Based on this data a graph displaying a comparison of the apparent solubilities was plotted. This graph takes into account, a 5\% error in calculation and plotting of the data which is shown below.

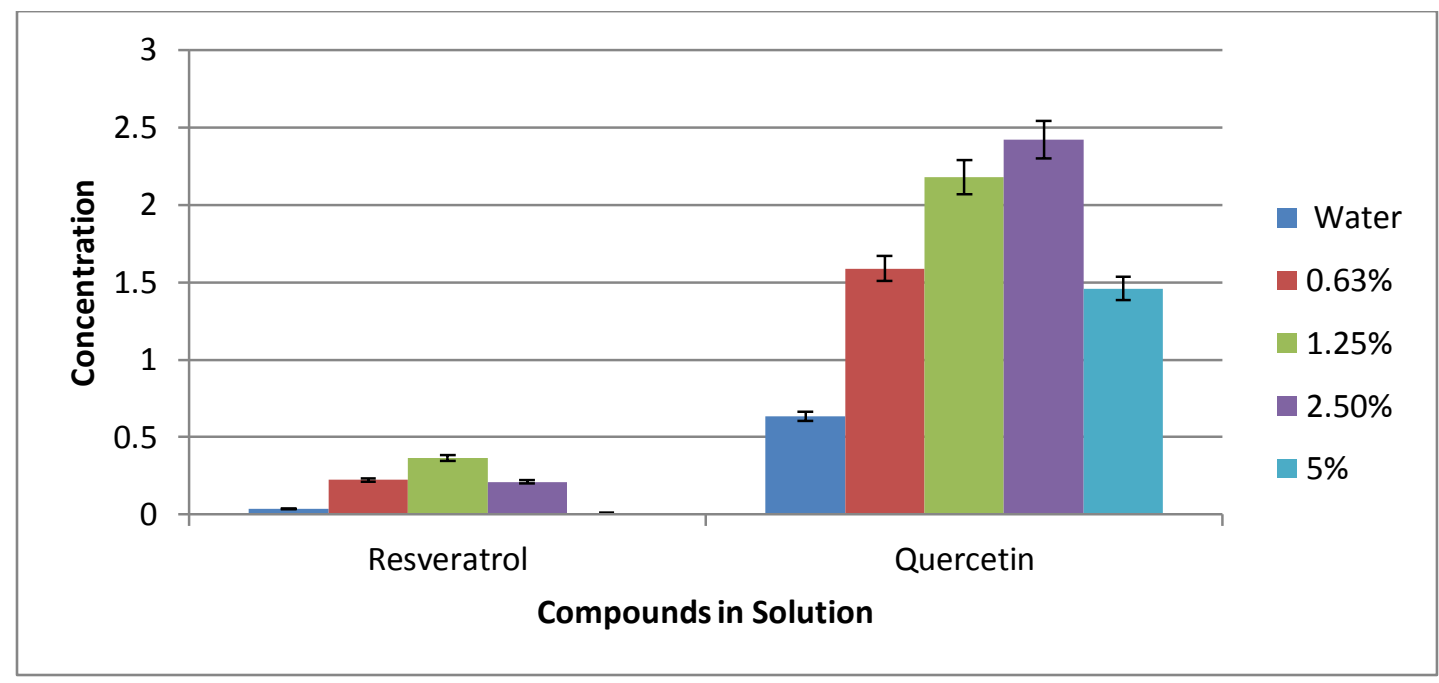

Figure 10: A comparison of the apparent solubilities of resveratrol and quercetin in water and in solutions of Soluplus® in water.

From this graph in figure 10, it appears that the apparent solubility of resveratrol increases with an increasing concentration of Soluplus ${ }^{\circledR}$ in water up to the extent of $1.25 \%$ Soluplus ${ }^{\circledR}$ by weight in water. Beyond this, there is a decrease in solubility or 
the solubility remains unchanged. In water, the solubility of resveratrol is lower than in solutions containing Soluplus ${ }^{\circledR}$ up to a concentration of $1.25 \%$ by weight.

Quercetin displays better solubility than resveratrol in water and shows the same trend as resveratrol although, its solubility decreases beyond a concentration of $2.5 \%$ by weight of Soluplus® in water.

A study was additionally conducted in order to determine the effect of micellar solubilization on the polyphenols in PBS solution. The samples were prepared as described in the methodology and were again analyzed by HPLC. Using the AUC values, the concentrations of the polyphenols in solution were determined and the following data was obtained.

\begin{tabular}{|l|l|l|l|l|}
\hline Compound & \multicolumn{3}{|c|}{ Solution } \\
\cline { 2 - 6 } & $10 \%$ & Water & $2 \mathrm{mg} / \mathrm{ml}$ & $16 \mathrm{mg} / \mathrm{ml}$ \\
& Soluplus in & & Soluplus in & Soluplus ${ }^{\circledR}$ in \\
& PBS & & PBS (below & PBS (above \\
& & & Soluplus ${ }^{\circledR}$ & Soluplus \\
& & & CMC) & CMC) \\
\hline Resveratrol & 0.00217 & 0.0376 & 0.0370 & 0.0610 \\
\hline Quercetin & 0.00284 & 0.0112 & 0.023 & 0.0053 \\
\hline
\end{tabular}

Table 5: Data obtained for the concentrations of polyphenols in different solutions of Soluplus ${ }^{\circledR}$ in PBS and in water 
Based on this data, a graph was plotted in order to compare the apparent solubilities of the polyphenols. A 5\% error was taken into consideration. The chart showing the comparative data is shown on the next page.

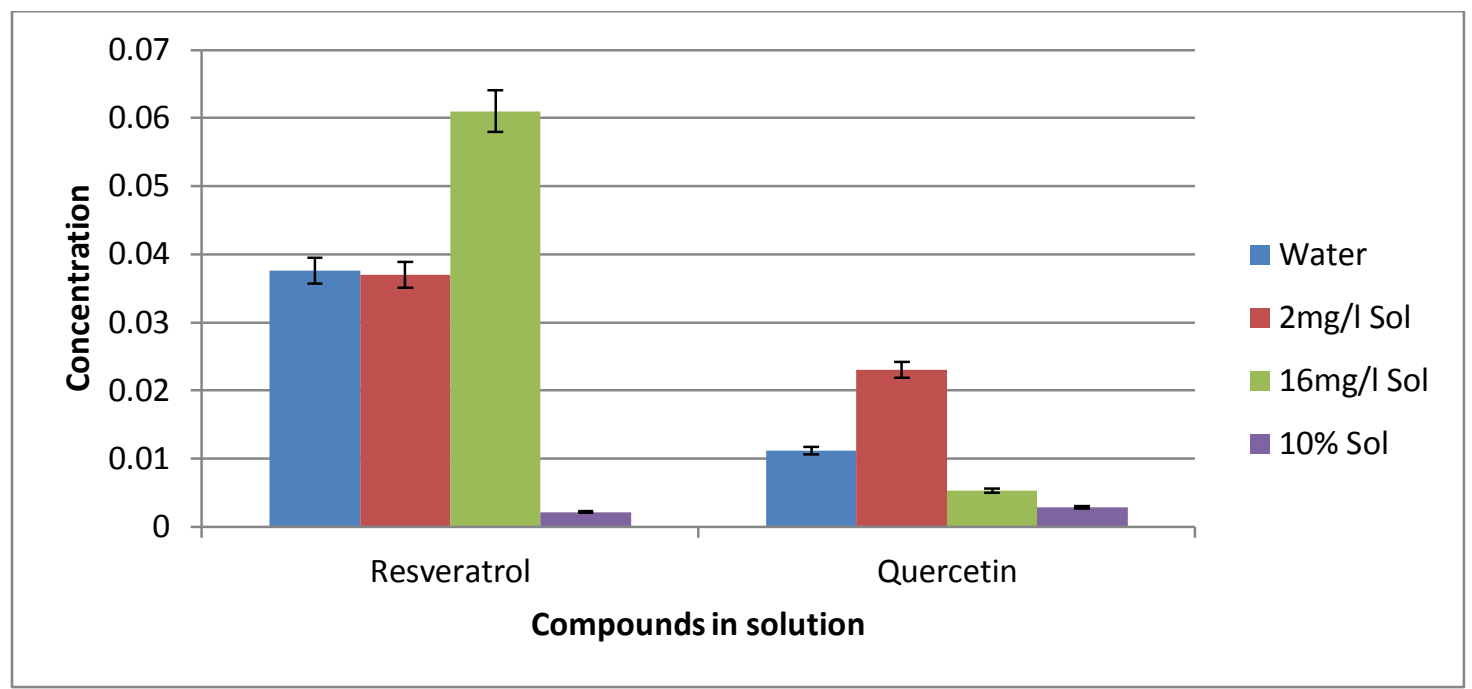

Figure 11: A chart showing the apparent solubilities of resveratrol and quercetin in water and in Soluplus ${ }^{\circledR}$ dissolved in PBS.

From this chart in figure 11, it is observed that there are differences in the trends of the apparent solubilities of resveratrol and quercetin. Resveratrol in water and in a $2 \mathrm{mg} / \mathrm{ml}$ solution of Soluplus ${ }^{\circledR}$ in PBS appear to have similar solubilization. In a concentration above the $\mathrm{CMC}$ of Soluplus ${ }^{\circledR}$, resveratrol has a major increase in its apparent solubility and in a saturated solution of Soluplus ${ }^{\circledR}$ in PBS, shows a drastic decrease in the apparent solubility as compared to the other solutions of resveratrol.

Quercetin shows improved solubility with a $2 \mathrm{mg} / \mathrm{ml}$ solution of Soluplus ${ }^{\circledR}$ while the apparent solubility shows a reduction at a concentration of $16 \mathrm{mg} / \mathrm{ml}$ of Soluplus ${ }^{\circledR}$ in PBS and in a saturated solution of Soluplus® in PBS. 
Interpretations:

Based on the data and the charts, it appears that Soluplus ${ }^{\circledR}$ improves the apparent solubility of resveratrol and quercetin. However, this occurs only up to a certain concentration of Soluplus ${ }^{\circledR}$ by weight in water. Beyond this concentration, Soluplus ${ }^{\circledR}$ does not have much effect on the solubility of the polyphenols.

Soluplus ${ }^{\circledR}$ is a polymeric surfactant and thus may improve the solubility of poorly soluble compounds in a solution by the formation of micelles. Quercetin displays an apparent improvement in its solubility below the CMC of Soluplus ${ }^{\circledR}$ as can be seen in the figure 11. This perhaps occurs due to intimate chemical interaction of quercetin with Soluplus ${ }^{\circledR}$ which probably results in improved wetting of quercetin. In other words, micellar solubilization is an important aspect of the solubilizing property of Soluplus ${ }^{\circledR}$. It is known that ions can play a role in the formation of micelles in a solution. Phosphate buffer solution was chosen in order to prevent the role of ions in forming micelles as it has a neutral $\mathrm{pH}$. Water de-ionized was chosen for a comparative study. Based on the data obtained for the concentrations of the polyphenols dissolved and the charts plotted as a result, it appears that solubilties of resveratrol and quercetin are fairly similar and also show small variations at these concentrations. The effect of micellar solubilization on Soluplus ${ }^{\circledR}$ can be seen on resveratrol at a concentration above the $\mathrm{CMC}(7.6 \mathrm{mg} / \mathrm{ml})$ of Soluplus ${ }^{\circledR}$ in $\mathrm{PBS}$. However, at a saturated concentration of Soluplus ${ }^{\circledR}$ in PBS, both resveratrol and quercetin show no drastic improvements in solubility. 
In conclusion, it can be said that Soluplus ${ }^{\circledR}$ does not significantly improve the solubility of the polyphenols in different solutions and at different concentrations. Micellar solubilization by Soluplus ${ }^{\circledR}$ has minor effects on the apparent solubility of the polyphenols but produces no drastic changes.

Study 2

The formulation of polyphenolic compounds into thin films for oral delivery.

Results

The study involved the formulation of thin films containing the polyphenolic compounds and Soluplus ${ }^{\circledR}$ dissolved in Dimethyl formamide (DMF). The formulation process was conducted as per the methodology described in the previous chapter. The films were placed on a glass plate for drying in a vacuum oven. Upon drying, they were removed from the oven and observed under the microscope. The observation revealed that resveratrol underwent re-crystallization on drying. This could be observed from the crystals of resveratrol present on the films. The films of resveratrol additionally displayed cracks and non-uniformity in certain spots. The films were thus, observed to be unstable.

Quercetin when formulated as a film with Soluplus® in DMF showed no visible undissolved particles. However, upon drying, the films appeared rough, non-uniform and developed cracks which could be seen with the naked eye. When observed under the microscope, these films showed traces of re-crystallization. 
Interpretation and conclusion

From the pharmaceutical formulation point of view, any finished product must remain stable and maintain its characteristics throughout its shelf-life. In the case of film formulations, common signs of an unstable product are breaking, cracking, and discoloration, release of fluids and re-crystrallization of the active constituents. When an insoluble or poorly soluble constituent is used in film formulations, adequate care is taken during the formulation process to prevent the precipitation or separation of the compound in solution. Re-crystallization indicates thermodynamic instability and can be initiated by the presence of ions or by chemical reactions with the adjuvant ingredients. It is undesired and reduces the activity of the formulation.

In the study conducted, it was observed that the polyphenols, resveratrol and quercetin could not be successfully formulated as films for oral delivery with Soluplus $®$. The films were brittle, showed signs of re-crystallization and were non-uniform. Additionally, the films displayed visible signs of instability such as cracking and breaking. Although, Soluplus ${ }^{\circledR}$ was used in adequate concentrations, it is observed that the film formulation of resveratrol and quercetin did not yield the desired results. Hence, it can be said that these polyphenols may be formulated into thin films for oral delivery using the applied concentration of Soluplus ${ }^{\circledR}$ using an alternative formulation comprising Soluplus® and other excipients. This may have afforded a pharmaceutically acceptable film but that determination is beyond the scope of this study. 
Study 3

The assay of content and determination of its uniformity of the hot-melt extruded mixtures of quercetin and resveratrol

Results

The determination of content uniformity is an important aspect of pharmaceutical formulations. Any changes in the chemical nature of the constituents of a formulation indicate that the quality of the final product will not be as per the desired standards. Thus, product stability may be affected. Changes in content uniformity also have an effect on the overall manufacturing process and quality at every step of the process is lost. The above experiment was conducted with the intent of analyzing the uniformity of the product by HPLC. The products were stirred in a mixture of water and methanol exhaustively before analysis was conducted. For a detailed study, a comparison of the chromatograms of three samples within a given mixture and comparisons between samples of different mixtures were carried out. The chromatograms obtained have been shown in the figures below. 


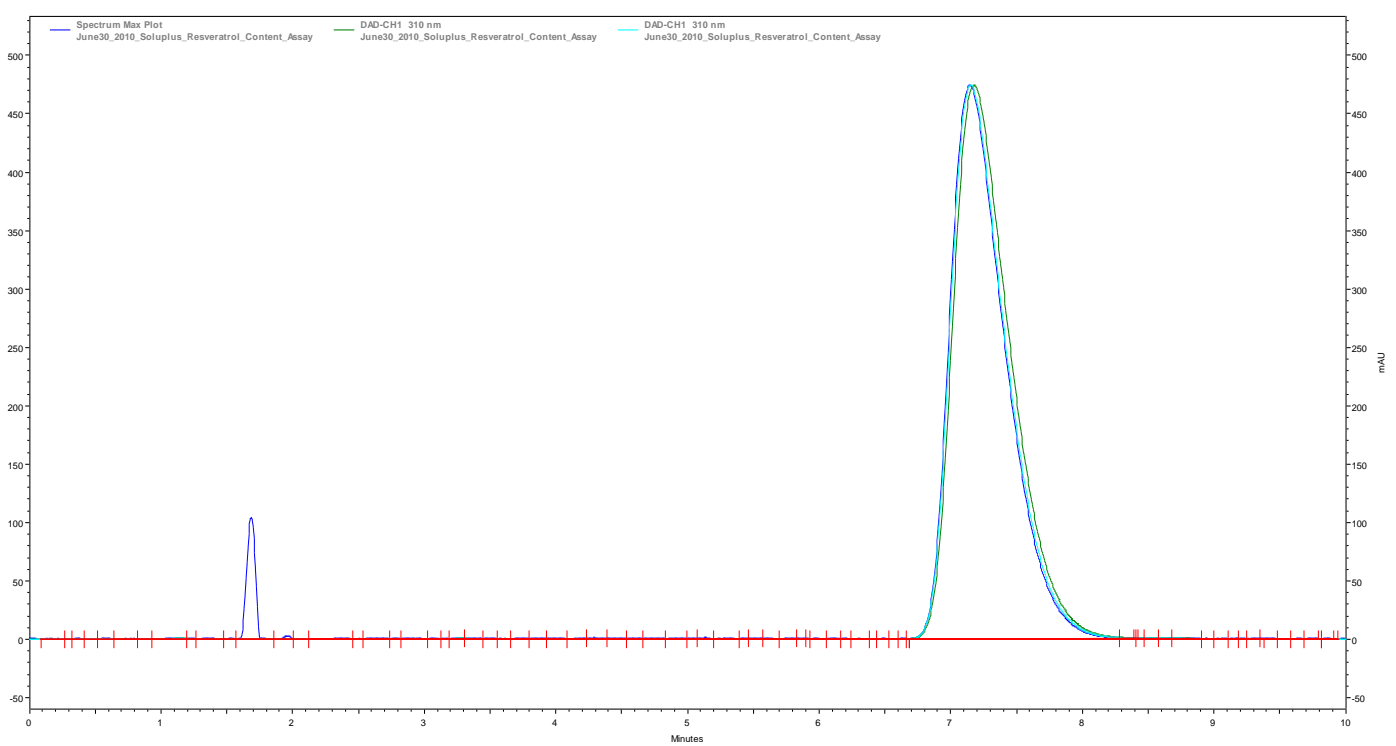

Figure 12: Multiple chromatogram view of 5\% by weight of resveratrol hot-melt extruded with Soluplus®

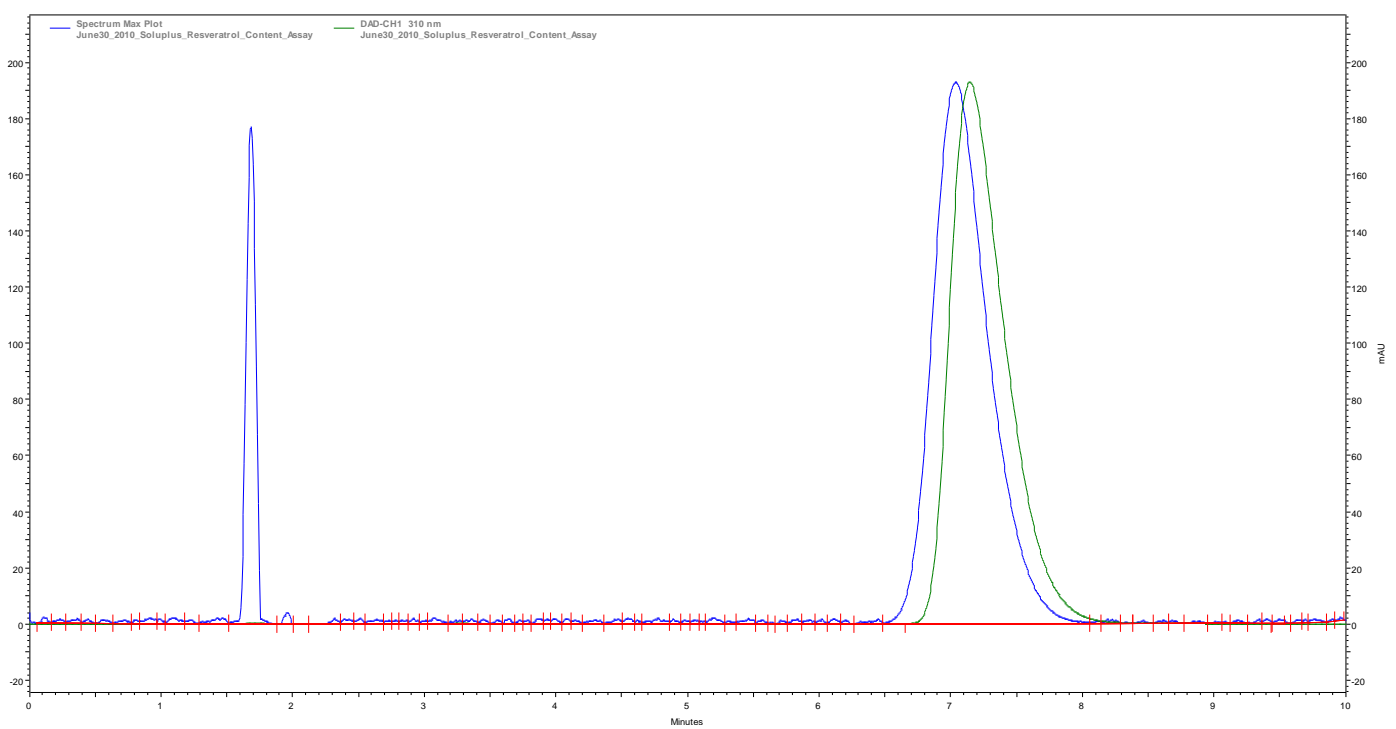

Figure 13: Multiple chromatogram view of two samples of resveratrol hot-melt extruded with Soluplus ${ }^{\circledR}$ at a concentration of $5 \%$ by weight of resveratrol and $2 \%$ by weight of resveratrol. 


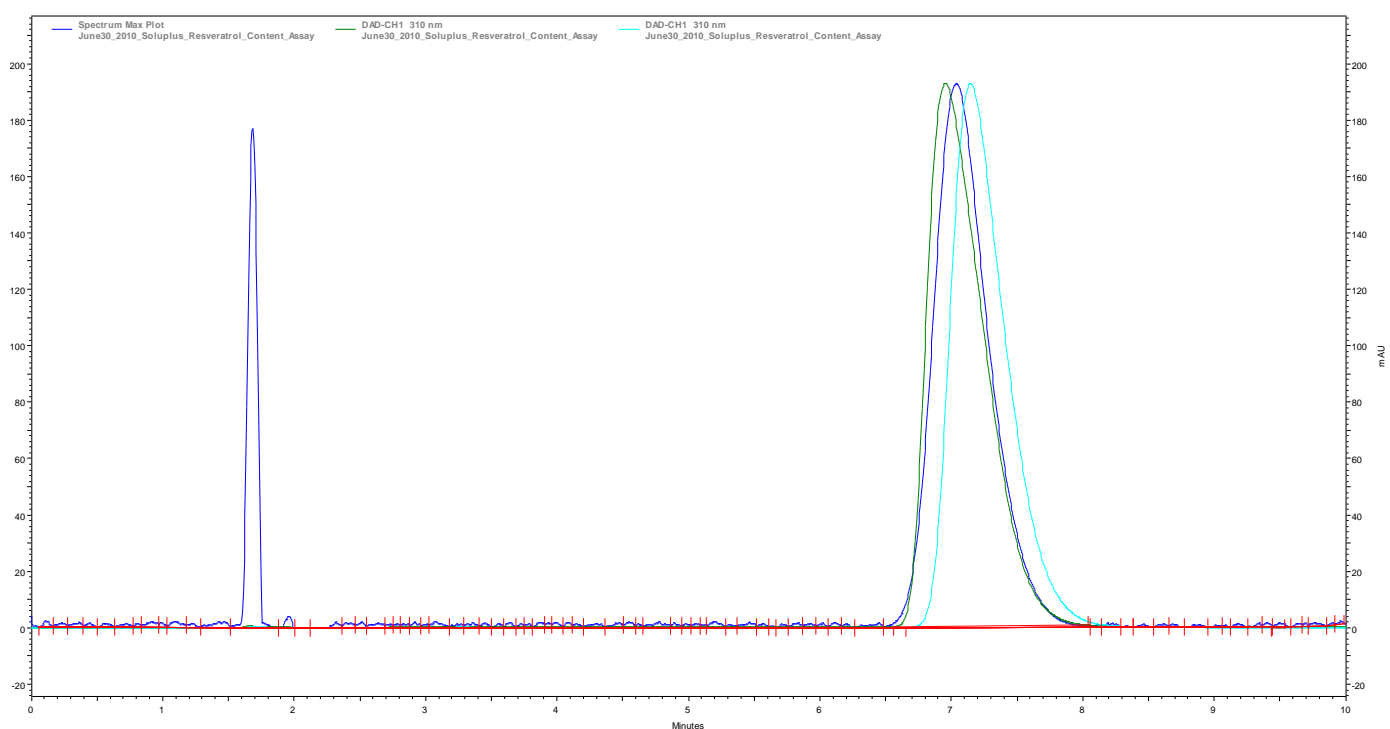

Figure 14: Multiple chromatogram view of the hot-melt extruded mixtures of resveratrol in Soluplus ${ }^{\circledR}$ in the concentrations of $2 \%$ and $5 \%$ by weight of resveratrol and the physical mixture of $0.5 \%$ by weight of resveratrol in Soluplus ${ }^{\circ}$

From the chromatograms above, it is observed that the time of retention for all the samples of resveratrol in Soluplus ${ }^{\circledR}$ lies between 7 to 8 minutes with peak values at approximately 7.1 minutes. Slight variations occur with minute differences in retention times and shifts in the peaks but are not of much concern. The AUC values vary depending on the concentration of resveratrol used in the mixture. For example, one sample of the $2 \% \mathrm{HME}$ resveratrol has an AUC value of 22382492 while a sample of the 5\% HME resveratrol has an AUC value of 54982055. AUC values help in determining the concentration of the compound under consideration in the mixture and the above values indicate the obvious fact that the 5\% HME resveratrol contains more resveratrol than the 2\% HME resveratrol. Another peak appears in most samples between 1 to 2 minutes and is a peak for the Soluplus ${ }^{\circledR}$ used in the sample.

Similarly, chromatograms were obtained for quercetin and have been shown below. 


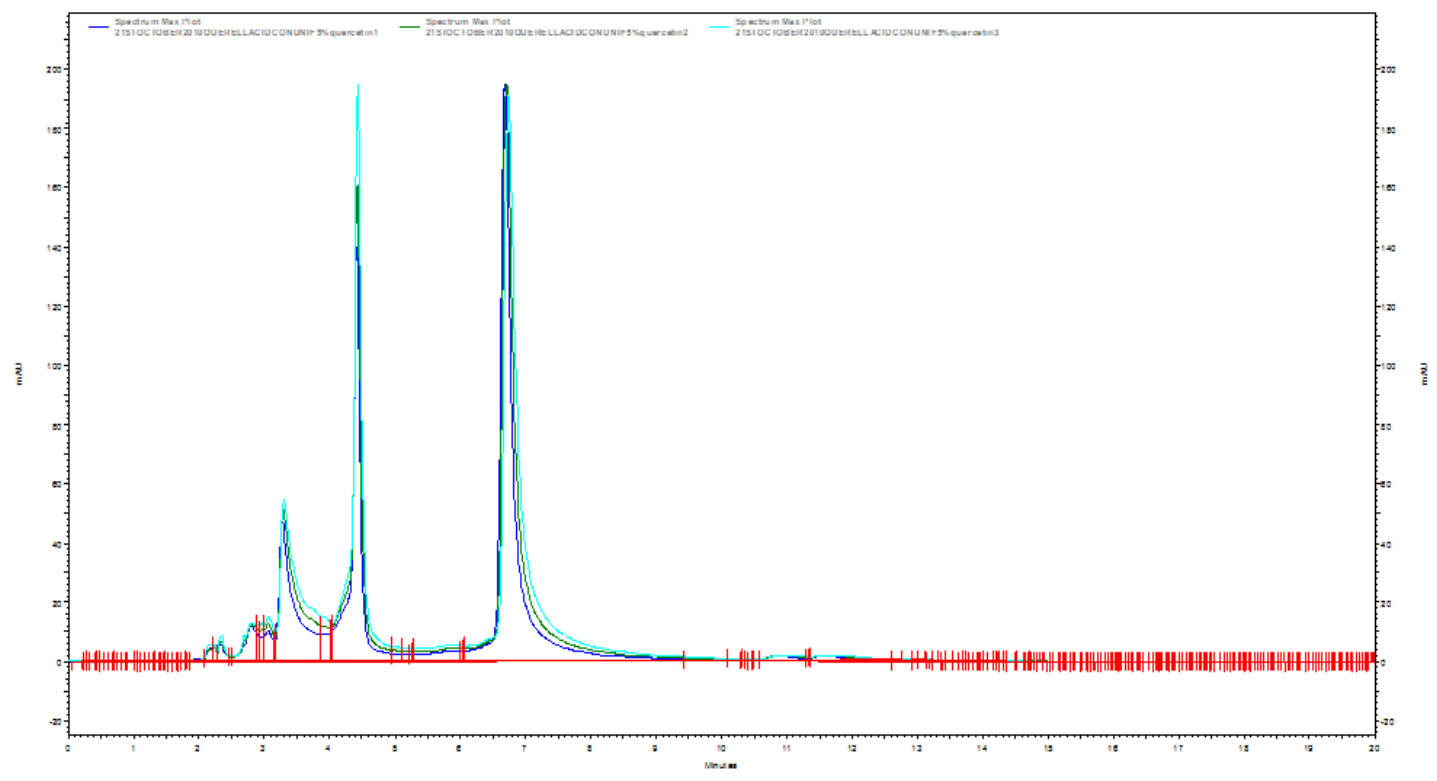

Figure 15: Multiple chromatogram view of three samples of Quercetin hot-melt extruded with Soluplus ${ }^{\circledR}$ at a concentration of $5 \%$ by weight of quercetin.

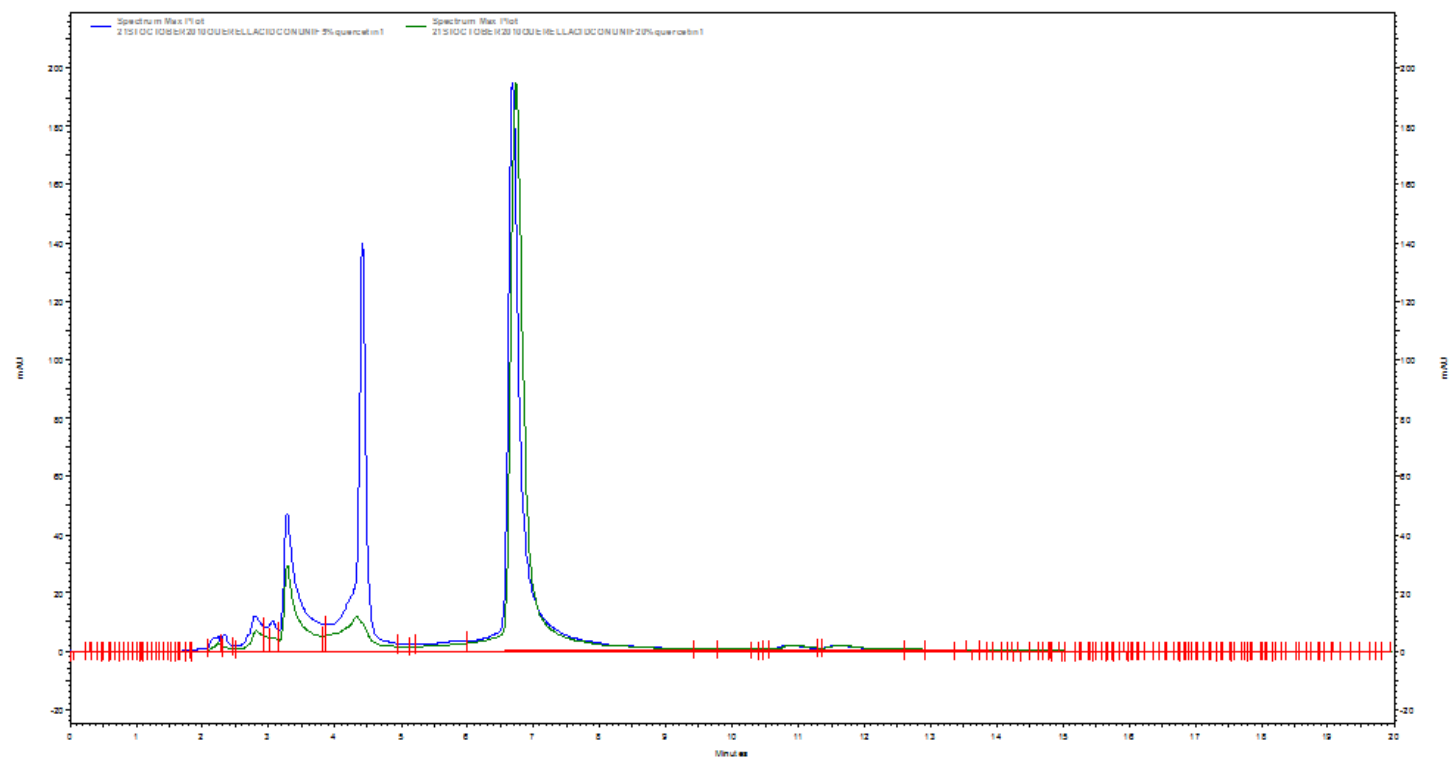

Figure 16: Multiple chromatogram view of two samples of Quercetin hot-melt extruded with Soluplus ${ }^{\circledR}$ at a concentration of $5 \%$ and $20 \%$ by weight of Quercetin. 


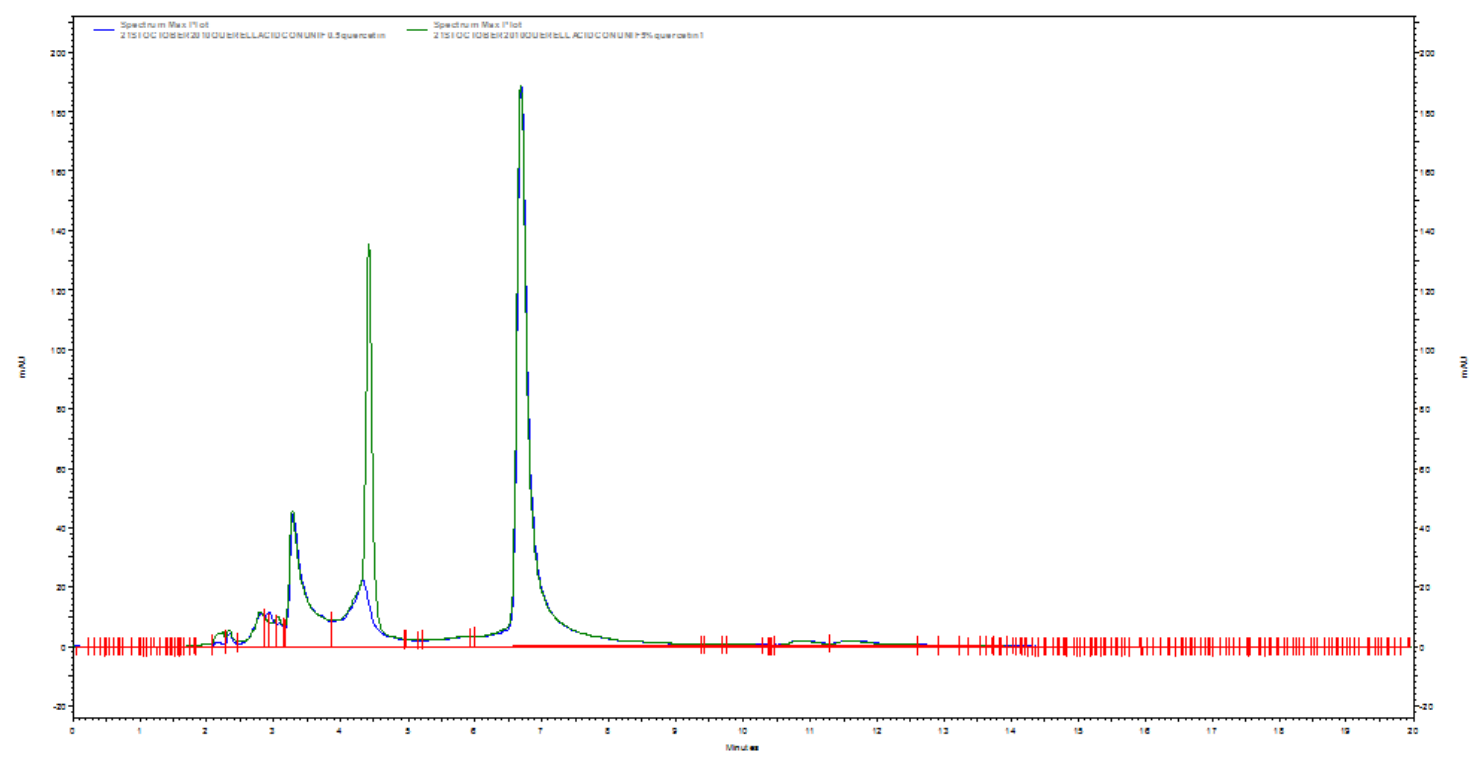

Figure 17: Multiple chromatogram view of a sample of Quercetin physically mixed with Soluplus $®$ at a concentration of $0.5 \%$ by weight of Quercetin and a sample of Quercetin hot-melt extruded with Soluplus $®$ at a concentration of $5 \%$ by weight of Quercetin.

From the chromatograms above, it is observed that the retention times for quercetin samples lie between 6 to 7 minutes. Most samples show peak absorption at a retention time of approximately 6.7 minutes. The peaks are superimposable as seen above with slight variations in some samples which are not of much concern. The AUC values obtained vary with the concentration of the quercetin present in the sample. For example, one sample of 5\% quercetin HME shows an AUC value of 4448672 while a sample of $20 \%$ quercetin HME shows an AUC value of 47792442. Additional peaks are observed in quercetin at a retention time of close to 4.5 minutes. These peaks are obtained due to the Soluplus ${ }^{\circledR}$ present in the sample. Similar peaks were obtained in the resveratrol samples and these were confirmed 
by running a pure sample of Soluplus ${ }^{\circledR}$ in $80 \%$ methanol and $95 \%$ methanol. These can be seen in the following figure.

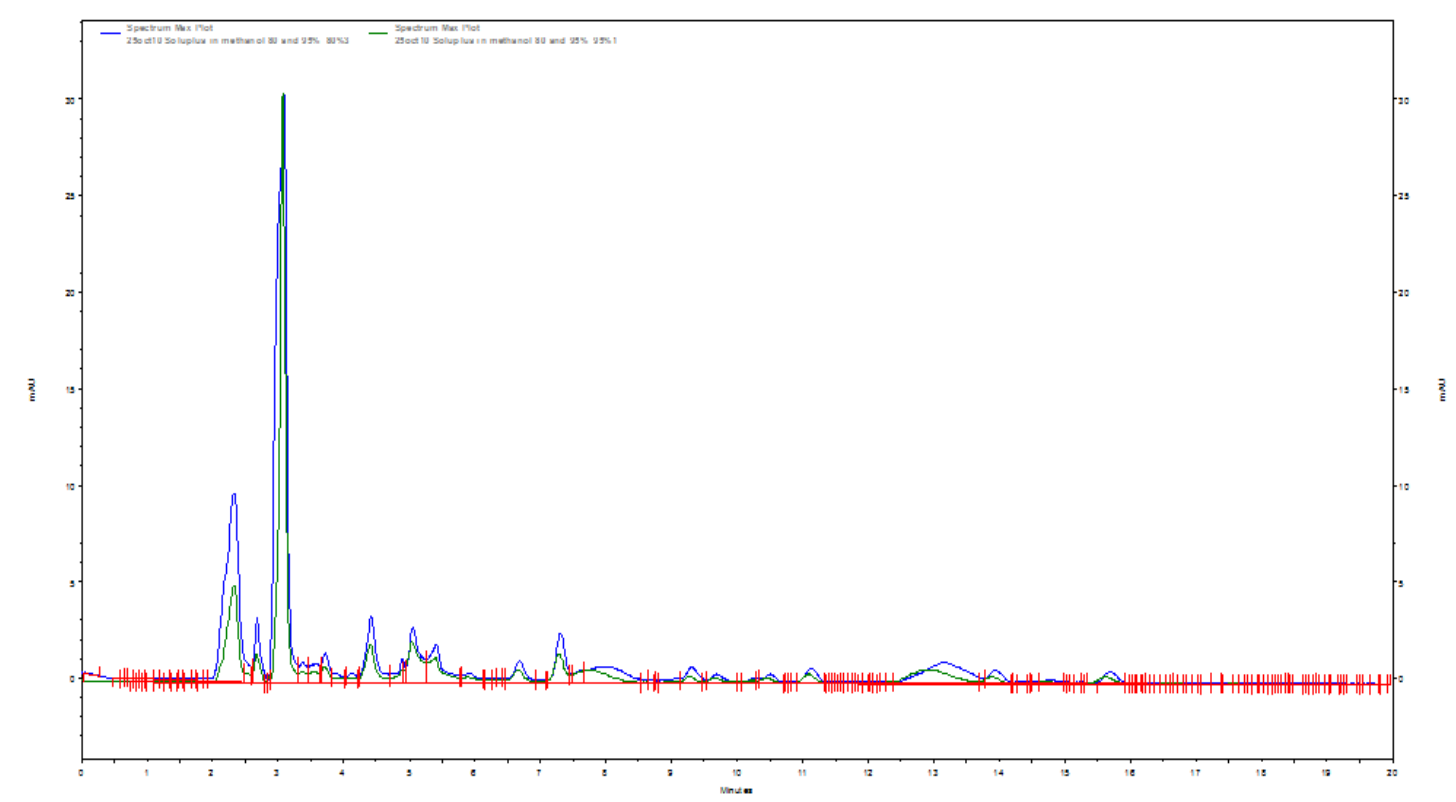

Figure 18: Multiple chromatogram view for a sample of Soluplus ${ }^{\circledR}$ dissolved in $80 \%$ methanol and another sample of Soluplus ${ }^{\circledR}$ dissolved in $95 \%$ methanol.

\section{Interpretation:}

The chromatograms demonstrate that the peaks are superimposable with minute shifts in the retention times in certain samples. For both resveratrol and quercetin, satisfactory results are obtained by HPLC analysis and it can be said the mixtures are uniform in their content. Sampling was carried out from different parts of the mixture and the similar chromatograms obtained indicated that the content of resveratrol and quercetin remained uniform throughout the mixture. This ensured that, the quality of the formulation produced will be of the desired standard and will not contain unequal distribution of the active constituents. The comparison with physical mixtures of 
resveratrol and quercetin in Soluplus ${ }^{\circledR}$ assisted in validating the findings of uniformity of content.

Study 4

A study of the dissolution characteristics of resveratrol in Capsules

Results

The study of dissolution of a dosage form provides an idea of the concentration of the drug released from the dosage form over a period of time. Thus, pharmaceutical dosage forms can be characterized depending upon the rate at which the drug is released from them. The above study was conducted in order to compare the release rates of resveratrol that was extruded with Soluplus ${ }^{\circledR}$ and a corresponding physical mixture of resveratrol and Soluplus ${ }^{\circledR}$ from capsules. The study was conducted as per the methodology described earlier and the following data was obtained. 


\begin{tabular}{|c|c|c|c|}
\hline \multirow[t]{2}{*}{ Time } & \multicolumn{3}{|c|}{ Cumulative percent released } \\
\hline & $\begin{array}{l}250 \mu \mathrm{m}-425 \mu \mathrm{m} \\
(\mathrm{HME})\end{array}$ & $\begin{array}{l}180 \mu \mathrm{m}-250 \mu \mathrm{m} \\
(\mathrm{HME})\end{array}$ & Physical mixture \\
\hline 0 & 0 & 0 & 0 \\
\hline 15 & 1.631 & 0.0416 & 10.454 \\
\hline 30 & 3.431 & 5.657 & 21.08 \\
\hline 45 & 3.84 & 11.555 & 21.24 \\
\hline 60 & 4.614 & 11.891 & 21.56 \\
\hline 90 & 5.67 & 28.57 & 21.76 \\
\hline
\end{tabular}

Table 6: Data obtained over 90 minutes for the mean cumulative percentage $( \pm \mathrm{SD})$ of content released from the three capsules of the hot-melt extruded mixture and similarly from the three capsules of the corresponding physical mixture

Based on the data obtained above a plot was created for the cumulative percentage released versus time for the capsules prepared from the HME mixtures and compared with the plots obtained for the corresponding physical mixture. This has been shown below.

The following figure shows the plot of the percentage cumulative release versus time for the HME mixture of 5\% resveratrol by weight and Soluplus ${ }^{\circledR}$ having a particle size between $180-250 \mu \mathrm{m}$ and the physical mixture of $5 \%$ resveratrol by weight and Soluplus ${ }^{\circledR}$. 


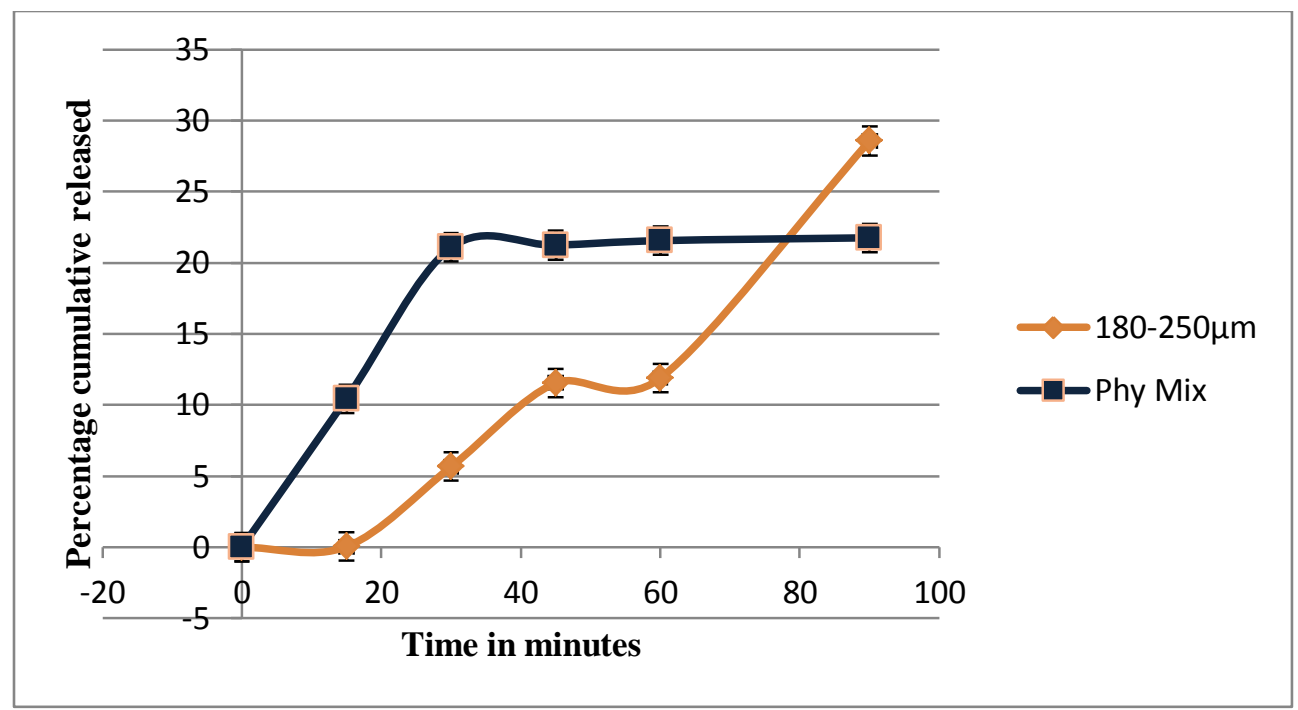

Figure 19: Plot of mean percentage cumulative released $( \pm$ SD) from capsules filled with HME mixture containing $5 \%$ resveratrol by weight with a particle size between 180-250 $\mu \mathrm{m}$ and Soluplus ${ }^{\circledR}$ and the corresponding physical mixture.

The figure above shows the plot of the mean percentage cumulative released versus time $( \pm \mathrm{SD})$ for the capsules containing the HME mixture of $5 \%$ by weight of resveratrol and Soluplus ${ }^{\circledR}$ having a particle size of $180-250 \mu \mathrm{m}$ along with the mean percentage cumulative released versus time $( \pm \mathrm{SD})$ for the capsules containing the physical mixture of $5 \%$ by weight of resveratrol and Soluplus®. It is observed that the rate of release of the capsules containing the physical mixture becomes constant after a period of 45 minutes. For the capsules containing the HME mixture of particle size $180-250 \mu \mathrm{m}$, the rate of release steadily increases up to 30 minutes and then slightly increases between 30 minutes and 45 minutes and then undergoes a rapid increase in the amount released towards the end of the measurement period.

The following figure shows the plot of the percentage cumulative release versus time for the HME mixture of 5\% resveratrol by weight and Soluplus® having a particle 
size between $180-250 \mu \mathrm{m}$ and the physical mixture of $5 \%$ resveratrol by weight and Soluplus ${ }^{\circledR}$.

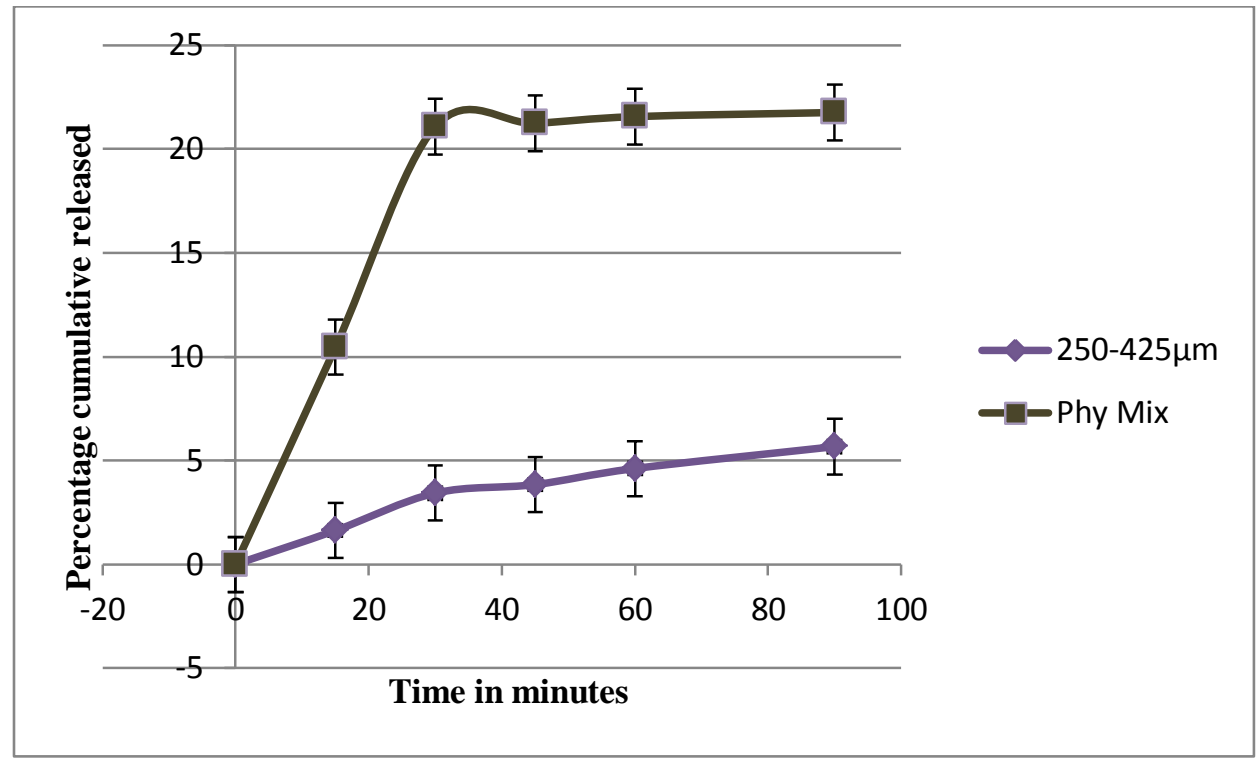

Figure 20: Plot of mean percentage cumulative released $( \pm$ SD) from capsules filled with HME mixture containing 5\% resveratrol by weight with a particle size between 250-425 $\mu \mathrm{m}$ and Soluplus ${ }^{\circledR}$ and the corresponding physical mixture.

The figure above shows the plot of the mean percentage cumulative released versus time $(\mathrm{SD} \pm 1)$ for the capsules containing the HME mixture of $5 \%$ by weight of resveratrol and Soluplus ${ }^{\circledR}$ having a particle size of $250-425 \mu \mathrm{m}$ along with the mean percentage cumulative released versus time $(\mathrm{SD} \pm 1)$ for the capsules containing the physical mixture of $5 \%$ by weight of resveratrol and Soluplus ${ }^{\circledR}$. Figure 12 shows that the rate of release of the capsules containing the physical mixture becomes constant after a period of 45 minutes as also seen in figure 11 . For the capsules containing the HME mixture of particle size $250-425 \mu \mathrm{m}$, the rate of release steadily increases up to 90 minutes. However, as seen from the data in table 3, in comparison with the mean 
percentage cumulative released for the capsules containing the HME mixtures with a particle size of $180-250 \mu \mathrm{m}$ and the capsules containing the physical mixture, the mean percentage cumulative released for the capsules containing the HME mixtures of 5\% resveratrol by weight with a particle size of $250-425 \mu \mathrm{m}$ is very low.

In the following figure, the plots from figures 11 and 12 have been summarized in a single plot.

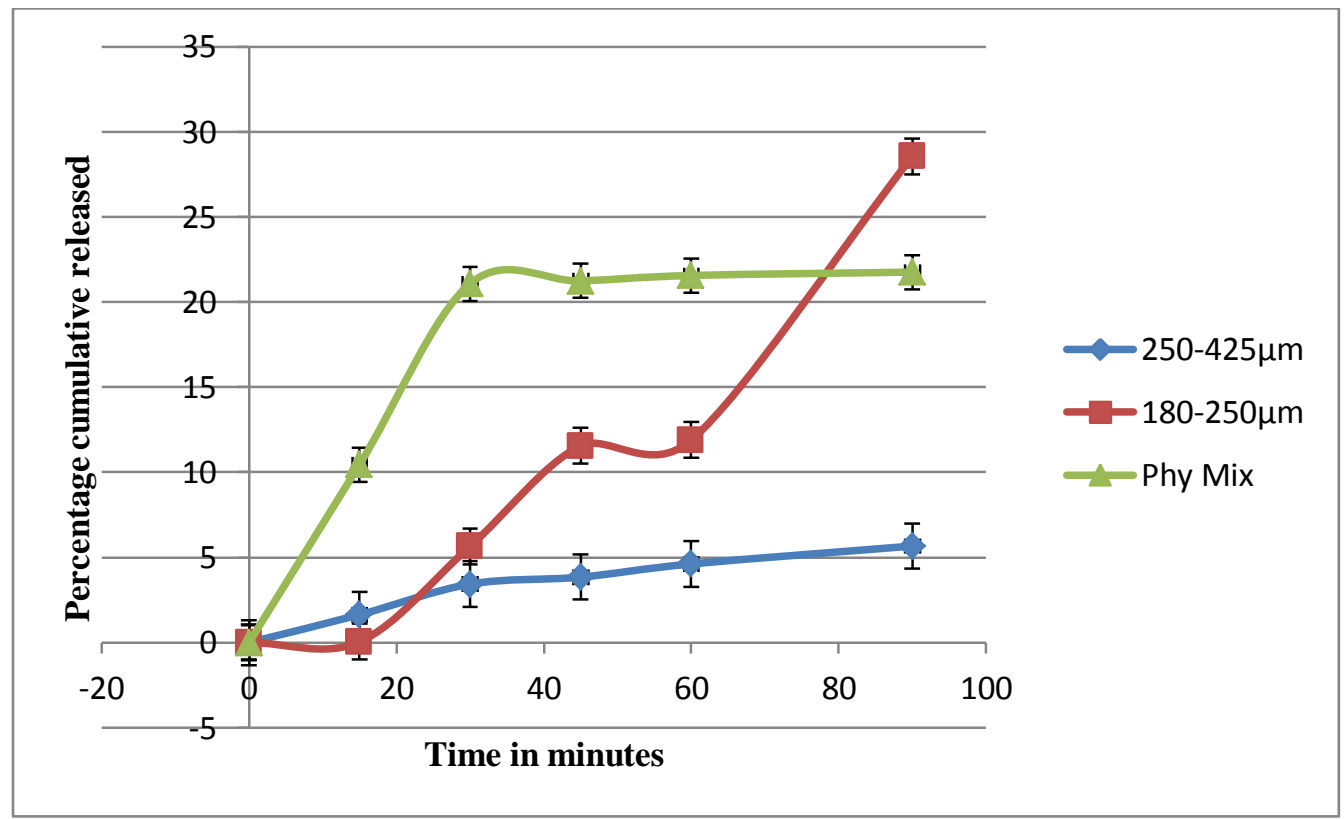

Figure 21: Plot of mean percentage cumulative released $( \pm$ SD) from capsules filled with HME mixtures containing $5 \%$ resveratrol by weight with a particle size between 180-250 $\mu \mathrm{m}$ and $250-425 \mu \mathrm{m}$ and Soluplus $®$ and similarly, the corresponding physical mixtures.

Interpretation:

From the plots and the data obtained above, it is observed that the release characteristics for each type of capsule are different. The release of the HME mixture 
of $5 \%$ by weight of resveratrol in Soluplus ${ }^{\circledR}$ having a particle size of $250-425 \mu \mathrm{m}$ occurs at a steady rate as can be seen in the plots and reaches a peak value of over 5\% of the total amount in the capsule at the end of the measurement period.

The release of the HME mixture of $5 \%$ by weight of resveratrol in Soluplus ${ }^{\circledR}$ having a particle size of $180-250 \mu \mathrm{m}$ occurs at a low rate between 0 and 15 minutes. Thereafter, there is a rapid increase in the amount released and again becomes slower between the 45 and 60 minute period. Towards the end of the measurement, there is a bulk release of the drug and reaches a final concentration of close to $30 \%$ of the total amount in the capsule.

In the case of the physical mixture, there is a rapid increase in the release of the drug up to 30 minutes. Beyond this period, the drug undergoes a nearly constant release and reaches a final concentration of close to $22 \%$ of the total amount present in the capsule.

It can thus be said that the release of resveratrol from the capsule filled with the HME mixture of $5 \%$ resveratrol by weight in Soluplus $®$ having a particle size of $250-425 \mu \mathrm{m}$ follows a slow and steady rate of release. The amount of drug released is also a very small percentage of the total amount present in the capsule at the end of the measurement period. On the other hand, the release of drug from the capsule containing the HME mixture of 5\% resveratrol by weight in Soluplus ${ }^{\circledR}$ with a particle size of $180-250 \mu \mathrm{m}$ occurs at a better rate than the $250-425 \mu \mathrm{m}$ particles. The rate slows down intermittently, but releases about $30 \%$ of the total amount of the drug in the capsule. Lastly, the physical mixture of $5 \%$ resveratrol by weight in Soluplus ${ }^{\circledR}$ 
follows an initial rapid release of the drug and then eventually shows a constant rate of release. The amount released at the end of 90 minutes is close to $22 \%$ of the total amount contained in the capsule. Summarily, the release of drug from the 5\% $\mathrm{HME}$ mixture having a particle size of $180-250 \mu \mathrm{m}$ has shown more satisfactory results as compared to the release from the $250-425 \mu \mathrm{m}$ particles and the release of the physical mixture of $5 \%$ by weight of resveratrol and Soluplus®. Perhaps, a smaller particle size of the mixture ensures better solubility properties and better dissolution characteristics.

\section{Study 5}

The characterization of molecular interactions between resveratrol and Soluplus and quercetin and Soluplus on hot-melt extrusion using Fourier transform infra-red spectroscopy

Results

FT-IR spectroscopic examination of the compounds was carried out as per the methodology described in the previous chapter using the Nicolet FT-IR. The spectra obtained were analyzed for the peak values of percentage transmittance over a period of 7 minutes on exposure to infra-red radiation. The spectra obtained were then compared in order to observe the similarities or differences present, to identify constituent functional groups and to determine the influence of the hot-melt extrusion process on the constituents. The spectra obtained are shown below. 


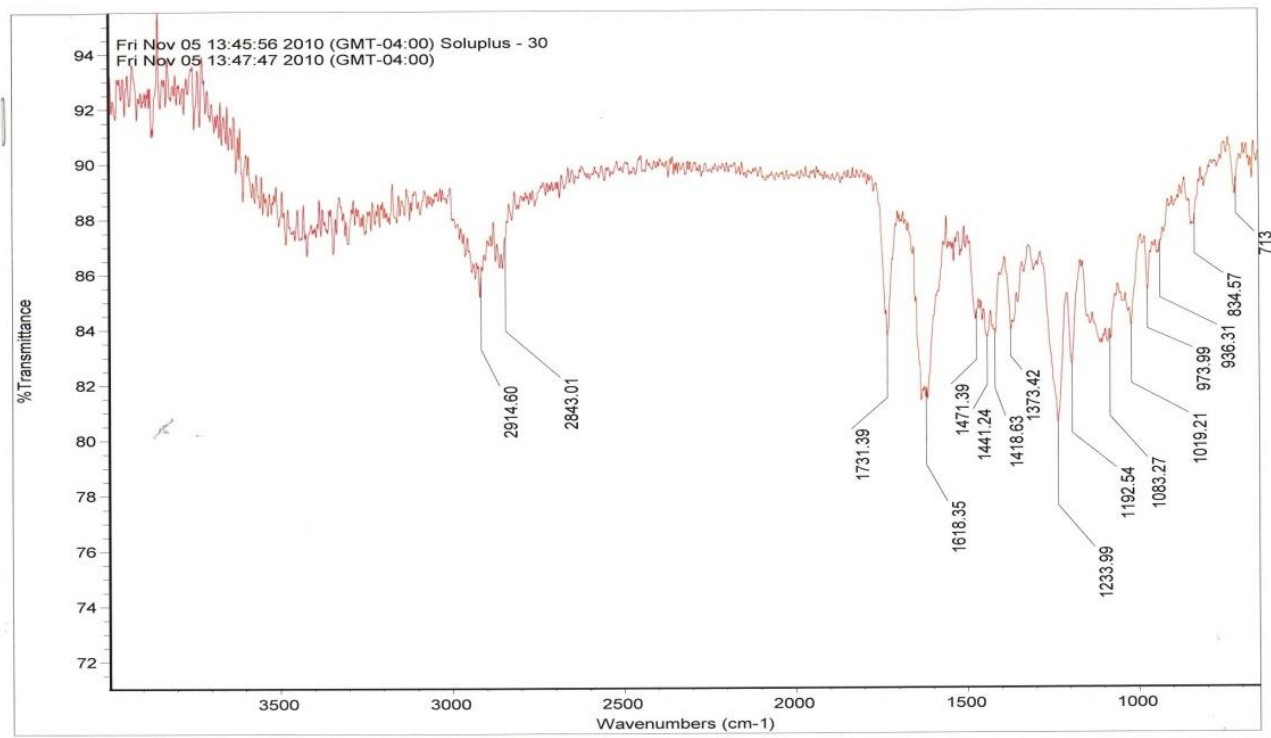

Figure 22: FT-IR spectrum of Soluplus ${ }^{\circledR}$

The spectrum shown above is of Soluplus ${ }^{\circledR}$. In this spectrum, broad and sharp peaks have been observed at $2900-2800 / \mathrm{cm}$ and strong peaks can be seen between 1732 and 1600/cm and between 1200 and 1100/cm. Small and weak peaks are observed beyond 975/cm. These are characteristic of certain functional groups present in the molecule.

Soluplus ${ }^{\circledR}$ is a polyvinyl caprolactam-polyvinyl acetate-polyethylene glycol graft copolymer. Thus, it has a lactam ring, acetate or ester linkage and alcohol groups in the molecule. The first step towards interpreting this spectrum is to look for characteristic $\mathrm{OH}, \mathrm{C}=\mathrm{O}$ and $\mathrm{NH}$ stretching which occurs in the region of $4000 / \mathrm{cm}$ to $900 / \mathrm{cm}$. These peaks can be seen in the spectrum in the figure above. According to the values from literature, acetates show strong peaks at close to $1800 / \mathrm{cm}$ and near $1200 / \mathrm{cm}$. There is a strong peak observed in the spectrum of Soluplus ${ }^{\circledR}$ at $1223 / \mathrm{cm}$ which suggests the presence of an acetate. Vinyl acetate shows a strong peak for carbonyl at $1776 / \mathrm{cm}$. 
Such a peak peak is observed at $1731 / \mathrm{cm}$ for this molecule. Lactams that are 6 or 7 membered display strong peaks at $\sim 1600 / \mathrm{cm}$ and in this spectrum, it is observed at $\sim 1618 / \mathrm{cm}$. Additionally, Soluplus ${ }^{\circledR}$ has free ether groups in the polyethylene glycol part of the molecule. These are capable of forming strong intramolecular bonds and thus, display broad stretching, as described in the literature at $\sim 3000 / \mathrm{cm}$. In the above spectrum, they have been shifted to a value of $2914.60 / \mathrm{cm}$ and $2843.01 / \mathrm{cm}$. The lactam undergoes $\mathrm{C}=\mathrm{O}$ stretching and thus shows a strong peak at close to $1650 / \mathrm{cm}$ region. The broad peaks observed between 800 and $700 / \mathrm{cm}$ in Soluplus ${ }^{\circledR}$ are due to the out-of-plane wagging that occurs in most lactam rings. Lastly, the vinyl peaks appear as medium peaks between $1450 / \mathrm{cm}$ and $1400 / \mathrm{cm}$ and as strong peaks between $1000 / \mathrm{cm}$ and $900 / \mathrm{cm}$ as seen in the spectrum above.

Further, FT-IR scans were carried out on resveratrol and quercetin. These are shown below.

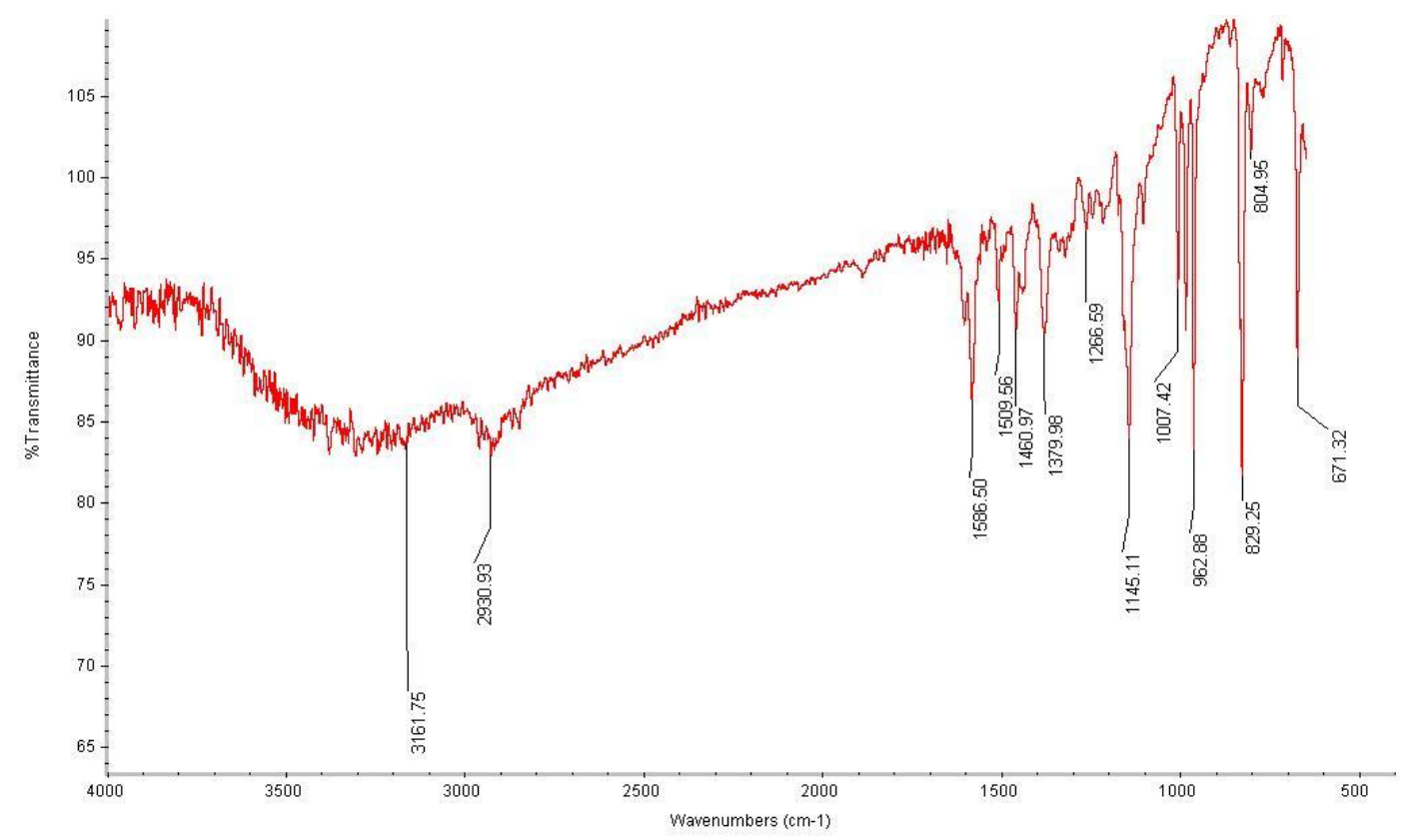

Figure 23: FT-IR spectrum of Resveratrol 
Resveratrol is a trans 3,5,4' trihydroxy stillbene. Structurally, it possesses three phenolic hydroxyl groups, two aromatic rings and an alkene. Since it is a polyphenolic compound, it shows a broad peak between $3200 / \mathrm{cm}$ to $2700 / \mathrm{cm}$. Similarly, weak peaks are noted between $2000 / \mathrm{cm}$ to $1600 / \mathrm{cm}$, indicating the presence of disubstituted benzene rings. Medium peaks are observed between $1700 / \mathrm{cm}$ to $1500 / \mathrm{cm}$ which again confirms the above finding. Strong transmittance is again observed between $1200 / \mathrm{cm}$ to $1000 / \mathrm{cm}$ and between $800 / \mathrm{cm}$ to $600 / \mathrm{cm}$, which confirms the presence of phenolic groups and substituted aromatic rings.

The strong transmittance bands at wavelengths between $900 / \mathrm{cm}$ and $675 / \mathrm{cm}$ are due to the bending of the ring $\mathrm{C}-\mathrm{H}$ bonds. These are the most prominent in determining the presence of aromatic rings. The next important characteristic band is that of the phenols and alcohols, which are observed due to the $\mathrm{O}-\mathrm{H}$ stretching and $\mathrm{C}-\mathrm{O}$ stretching. These vibrations are coupling vibrations that depend upon the adjacent groups. In the case of phenols, broad peaks are observed between $3200 / \mathrm{cm}$ to $3000 / \mathrm{cm}$ due to the intermolecular hydrogen bonding and $\mathrm{O}-\mathrm{H}$ stretching. In plane bending of $\mathrm{C}-\mathrm{H}$ bonds causes the strong transmittance to appear close to $1000 / \mathrm{cm}$. Lastly, doublet bands are observed between $1580 / \mathrm{cm}$ and $1400 / \mathrm{cm}$. These are due to C-C stretching within the ring.

The following figure shows the transmittance spectrum of quercetin. 


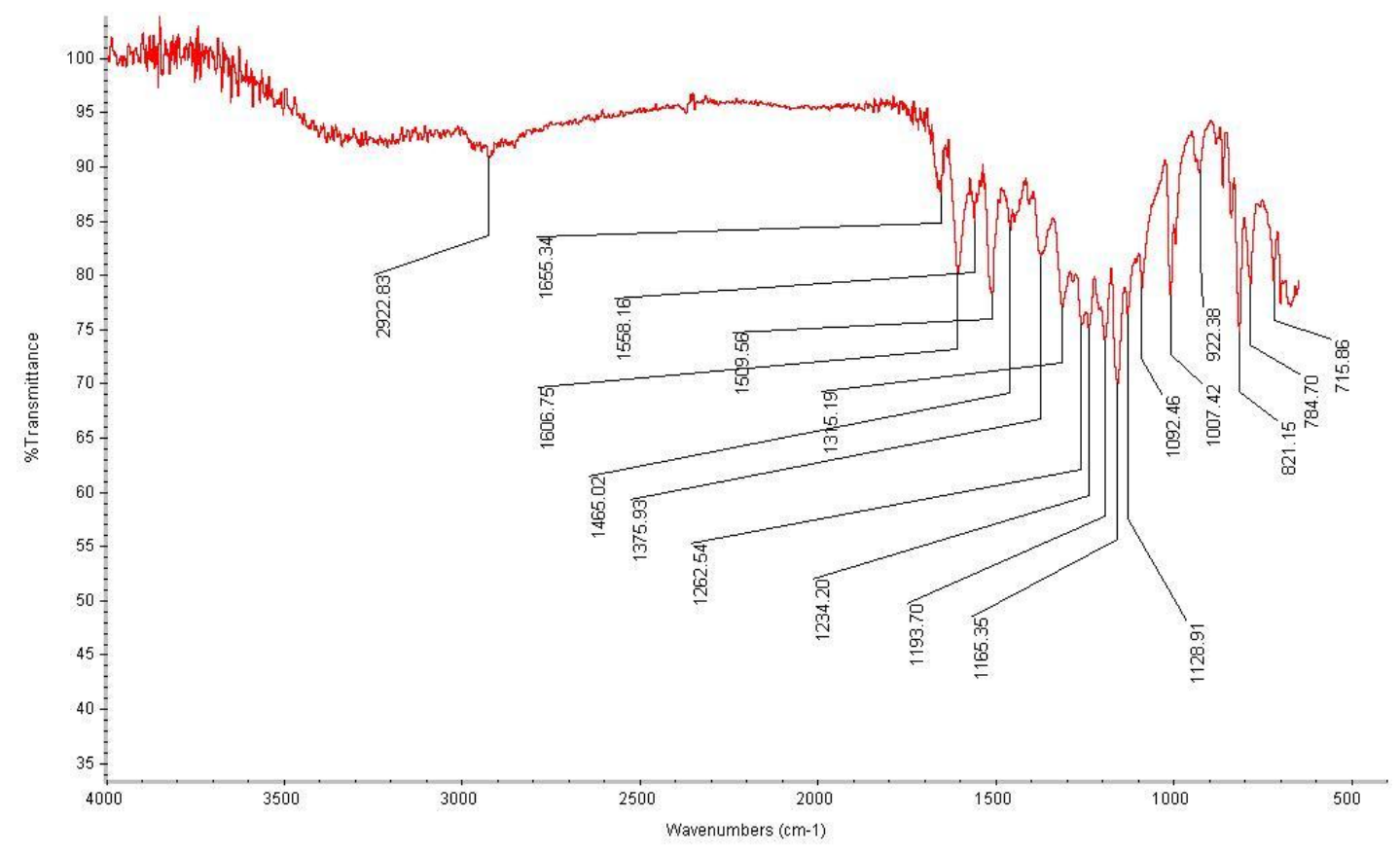

Figure 24: FT-IR spectrum of Quercetin

In the spectrum of quercetin, a broad peak is observed between $3100 / \mathrm{cm}$ and $2700 / \mathrm{cm}$. Strong peaks appear between $900 / \mathrm{cm}$ and $700 / \mathrm{cm}$ while medium peaks are observed between $1400 / \mathrm{cm}$ and $1160 / \mathrm{cm}$. Structurally, quercetin is 2-(3,4-dihydroxyphenyl)3,5,7-trihydroxy-4H-chromen-4-one. Thus, a characteristic transmittance band for a ketone would be observed due to the $\mathrm{C}=\mathrm{O}$ stretching. The aromatic conjugated ketones often display an absorption spectrum that is identified by a strong band close to $1600 / \mathrm{cm}$ and medium bands between $1400 / \mathrm{cm}$ and $1200 / \mathrm{cm}$, which occurs due to a delocalization of electrons $(\mathrm{Pi})$ that reduces the $\mathrm{C}=\mathrm{O}$ character. The remaining characteristic wavelengths of transmittance are similar to those observed in resveratrol. These include the bands for phenols and aromatic rings. The disubstituted benzene ring bands are observed between $1200 / \mathrm{cm}$ and $1000 / \mathrm{cm}$ and the stronger bands between $800 / \mathrm{cm}$ and $600 / \mathrm{cm}$ due to the $\mathrm{C}-\mathrm{H}$ stretching within the ring. 
Based on these spectra, a comparative study was carried out with the spectra obtained from the hot-melt extruded and physical mixtures of the polyphenols in Soluplus ${ }^{\circledR}$. The following figure shows the FT-IR spectrum of the hot-melt extruded mixtures of resveratrol in Soluplus ${ }^{\circledR}$.

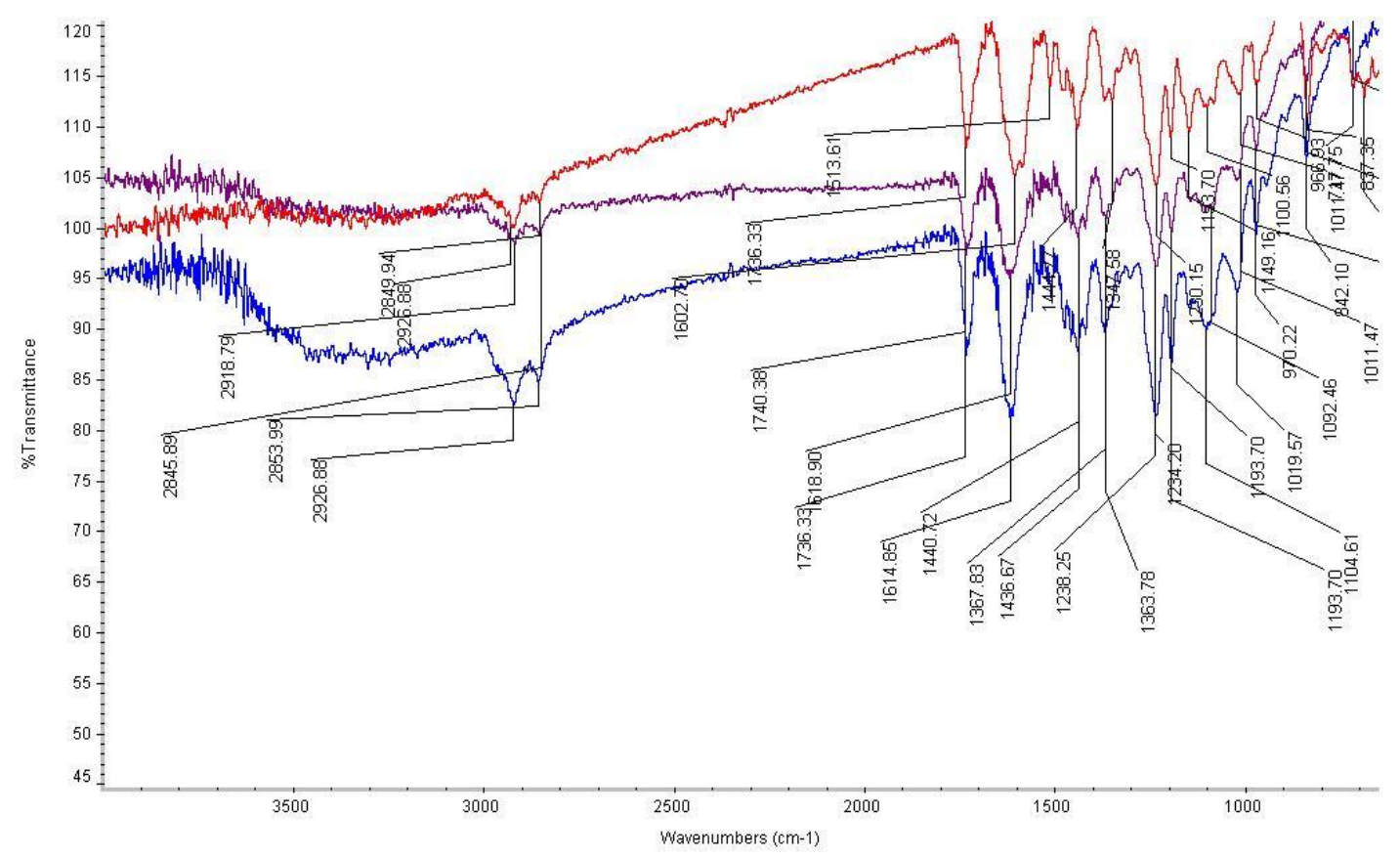

Figure 25: FT-IR spectra of the hot-melt extruded mixtures of resveratrol and Soluplus $®$ (Blue: $20 \%$ mixture, purple: $2 \%$ mixture and red: $5 \%$ mixture)

The above spectrum is a combined overlay view of the HME mixtures of resveratrol in Soluplus®. The spectra are superimposable as can be observed. The peaks are consistent for all the mixtures and appear at the same wavelengths.

The spectrum shows broad peaks observed with both resveratrol and Soluplus ${ }^{\circledR}$ between $3000 / \mathrm{cm}$ and $2700 / \mathrm{cm}$ for phenols but are closer to the peaks obtained with Soluplus ${ }^{\circledR}$. The other striking similarity is the appearance of strong peaks at close to 
$1735 / \mathrm{cm}$, the peak for vinyl acetate, in these melt extruded mixtures which are similar to the strong peak obtained in Soluplus ${ }^{\circledR}$. Further, the sharp and strong peaks obtained for resveratrol between $900 / \mathrm{cm}$ and $700 / \mathrm{cm}$ have been reduced to smaller medium sized peaks in these mixtures. The appearance of a sharp peak at close to $1600 / \mathrm{cm}$ is characteristic of $\mathrm{C}=\mathrm{O}$ in aromatic ketones. The sharp peak for resveratrol, seen at $1145 / \mathrm{cm}$ also has disappeared in this spectrum and a medium peak observed at $1193 / \mathrm{cm}$ is similar to the peak obtained in Soluplus ${ }^{\circledR}$. Thus, it can be said that hotmelt extrusion of resveratrol with Soluplus ${ }^{\circledR}$ has shown that the resultant product undergoes chemical changes to display functional group characteristics similar to Soluplus®. This finding was further confirmed when the spectra of the hot-melt extruded mixture of resveratrol in Soluplus ${ }^{\circledR}(20 \%)$ and its corresponding physical mixture were compared. 


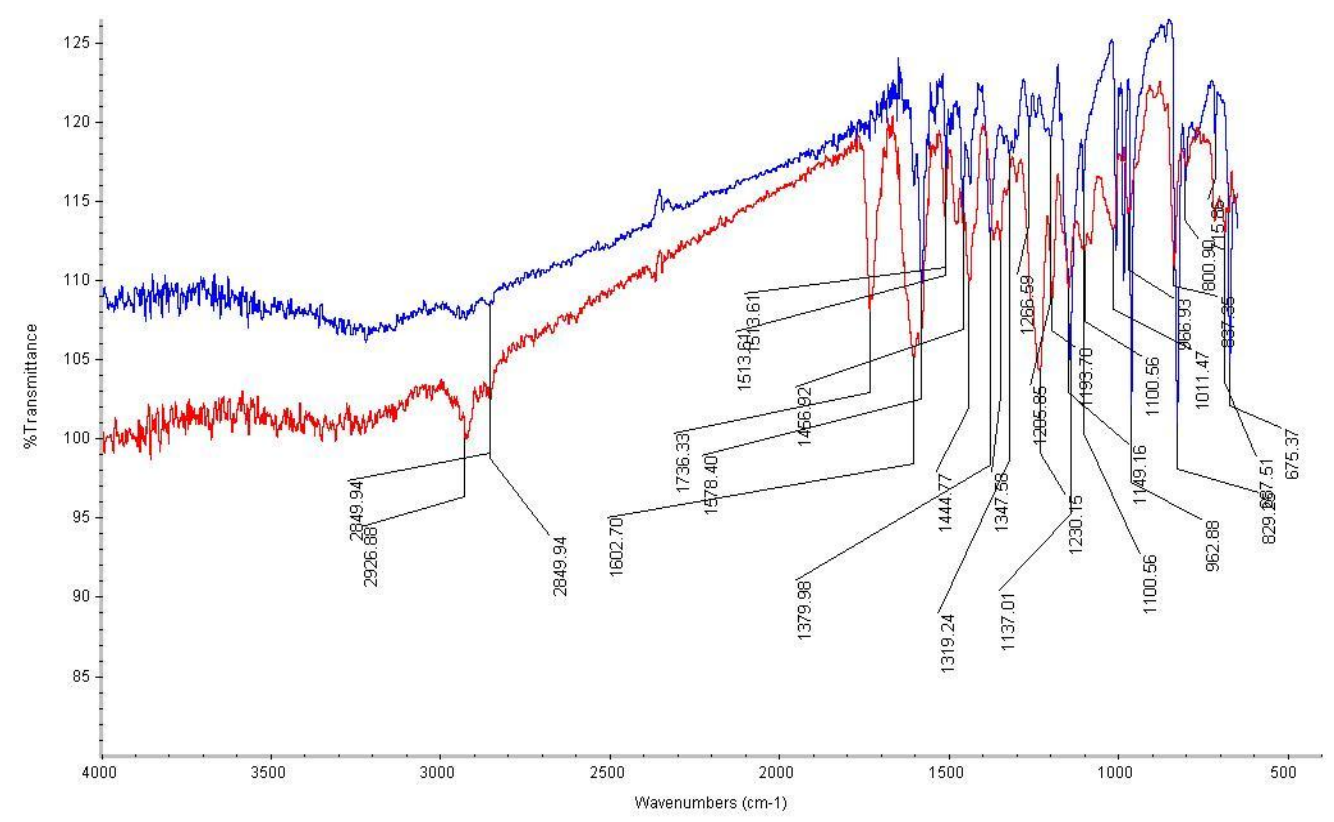

Figure 26: FT-IR spectra of a hot-melt extruded mixture of resveratrol in Soluplus® $20 \%$ by weight and a physical mixture of $20 \%$ resveratrol in Soluplus® (Blue: Physical mixture and red: HME mixture)

In the spectra of the physical mixture of resveratrol and Soluplus®, peaks of both compounds are observed. The hot-melt extruded mixture shows peaks more consistent with Soluplus $®$ and the sharp peaks observed for the physical mixture at $962 / \mathrm{cm}$, $829 / \mathrm{cm}$ and $675 / \mathrm{cm}$ are not observed in the hot - melt extruded mixture. A final confirmation of this finding is the superposition of the spectra of resveratrol HME $2 \%$ and Soluplus ${ }^{\circ}$ blank as seen in the figure below. 


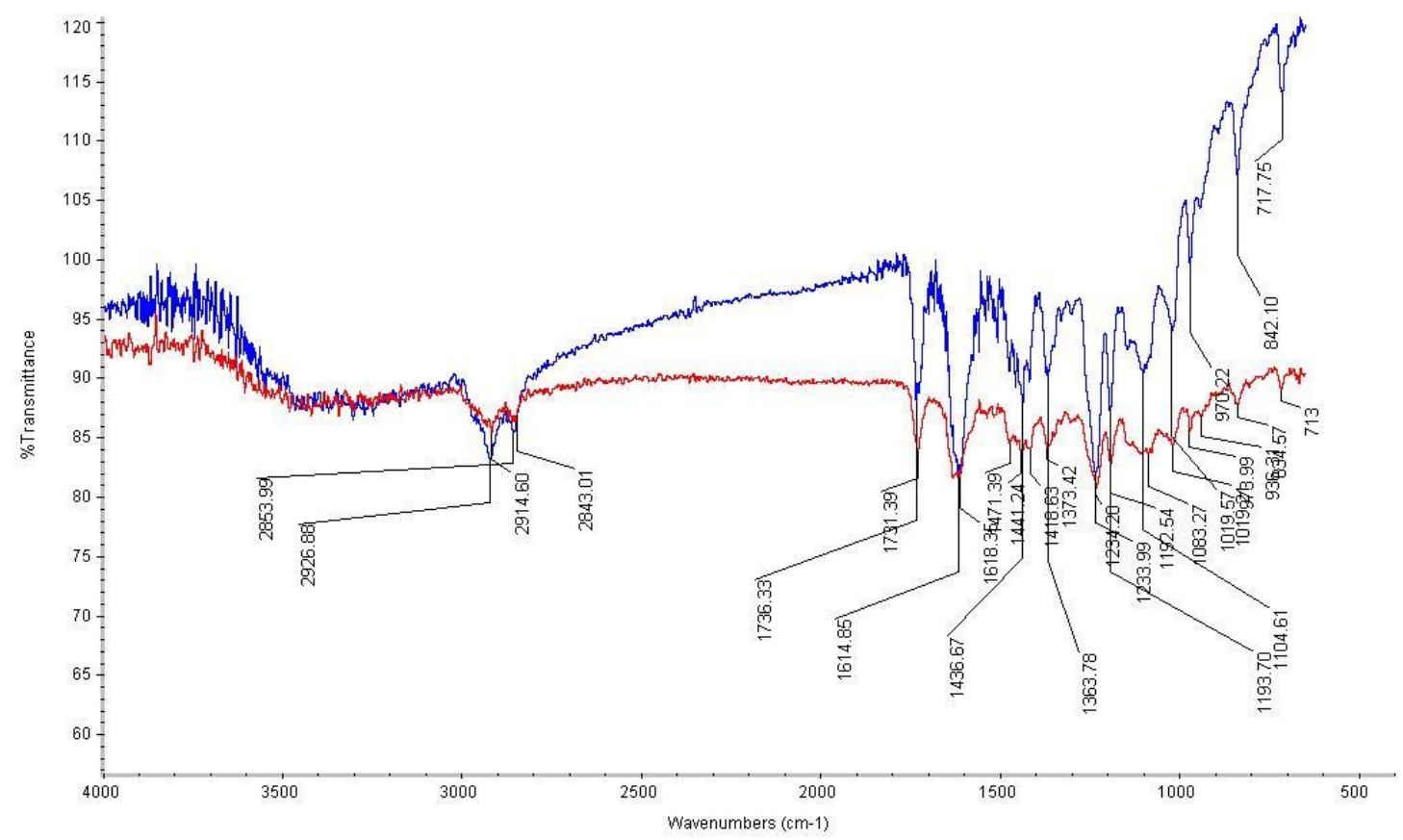

Figure 27: FT-IR spectra of Soluplus ${ }^{\circledR}$ and the HME mixture of $2 \%$ resveratrol by weight in Soluplus ${ }^{\circledR}$ (Blue: Soluplus ${ }^{\circledR}$ and red: HME mixture)

As can be seen from the figure, the spectra are almost superimposable with minute differences. The sharp peaks of Soluplus $®$, seen at $842 / \mathrm{cm}$ and $717 / \mathrm{cm}$ have now been reduced to medium sized peaks in the HME mixture.

Shown below are the spectra of the HME mixtures of quercetin and Soluplus ${ }^{\circledR}$ 


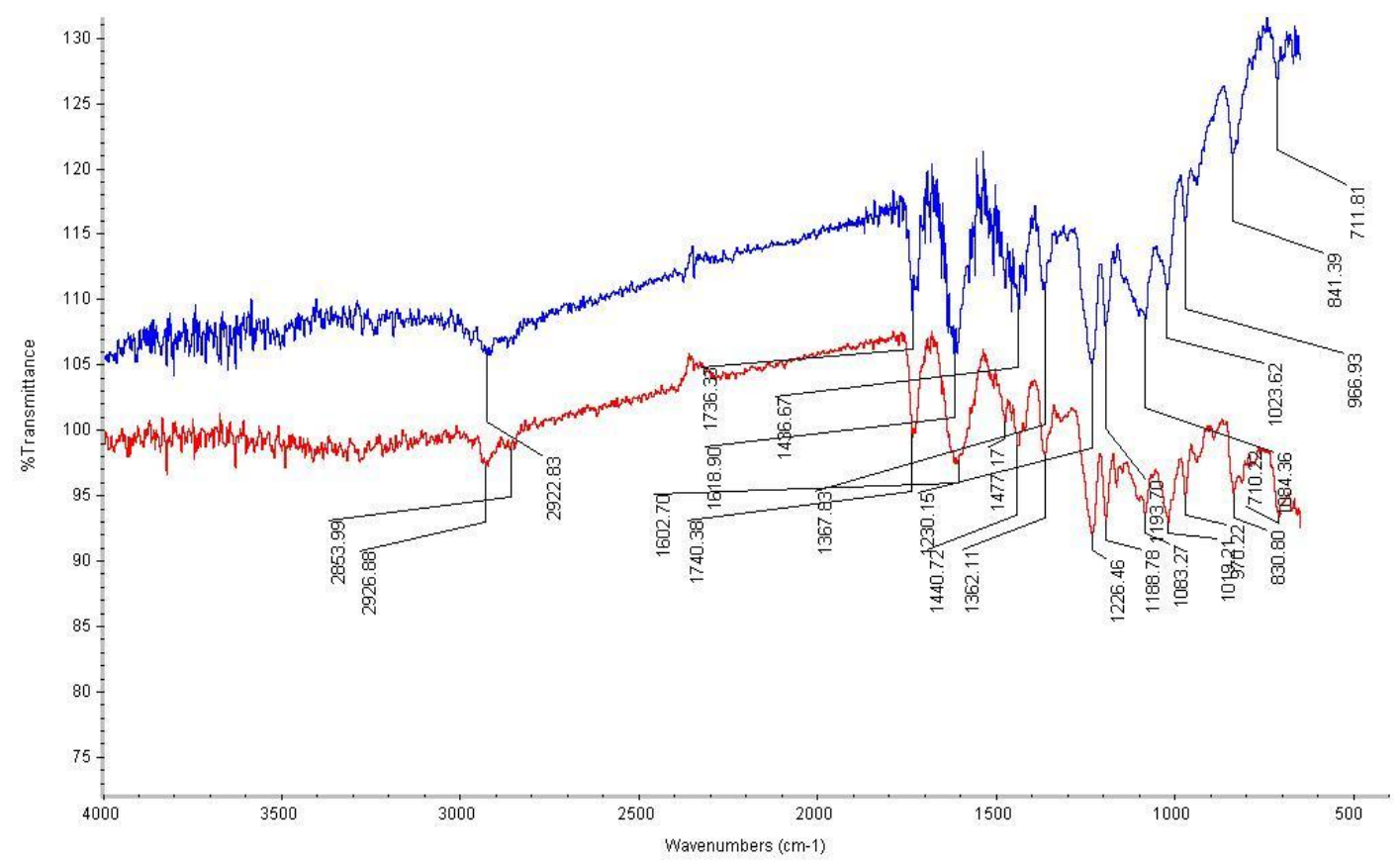

Figure 28: FT-IR spectra of the HME mixtures of both weight fractions of quercetin and Soluplus ${ }^{\circledR}$ (Blue: $20 \%$ by weight of quercetin in Soluplus ${ }^{\circledR}$ and red: $5 \%$ by weight of quercetin in Soluplus $®$ )

The peaks obtained for the HME mixtures are again almost superimposable for quercetin extruded with Soluplus ${ }^{\circledR}$. The sharp peaks obtained at $1740 / \mathrm{cm}$, $1602 / \mathrm{cm}$ and $1226 / \mathrm{cm}$ for the $5 \%$ mixture, are similar to the peaks obtained at $1736 / \mathrm{cm}, 1618 / \mathrm{cm}$ and $1230 / \mathrm{cm}$ for the $20 \%$ mixture. Thus, the functional group characteristics of both mixtures are similar.

The following figure displays the spectra of the HME mixture of 5\% by weight of quercetin in Soluplus ${ }^{\circledR}$ and the corresponding physical mixture of quercetin in Soluplus®. 


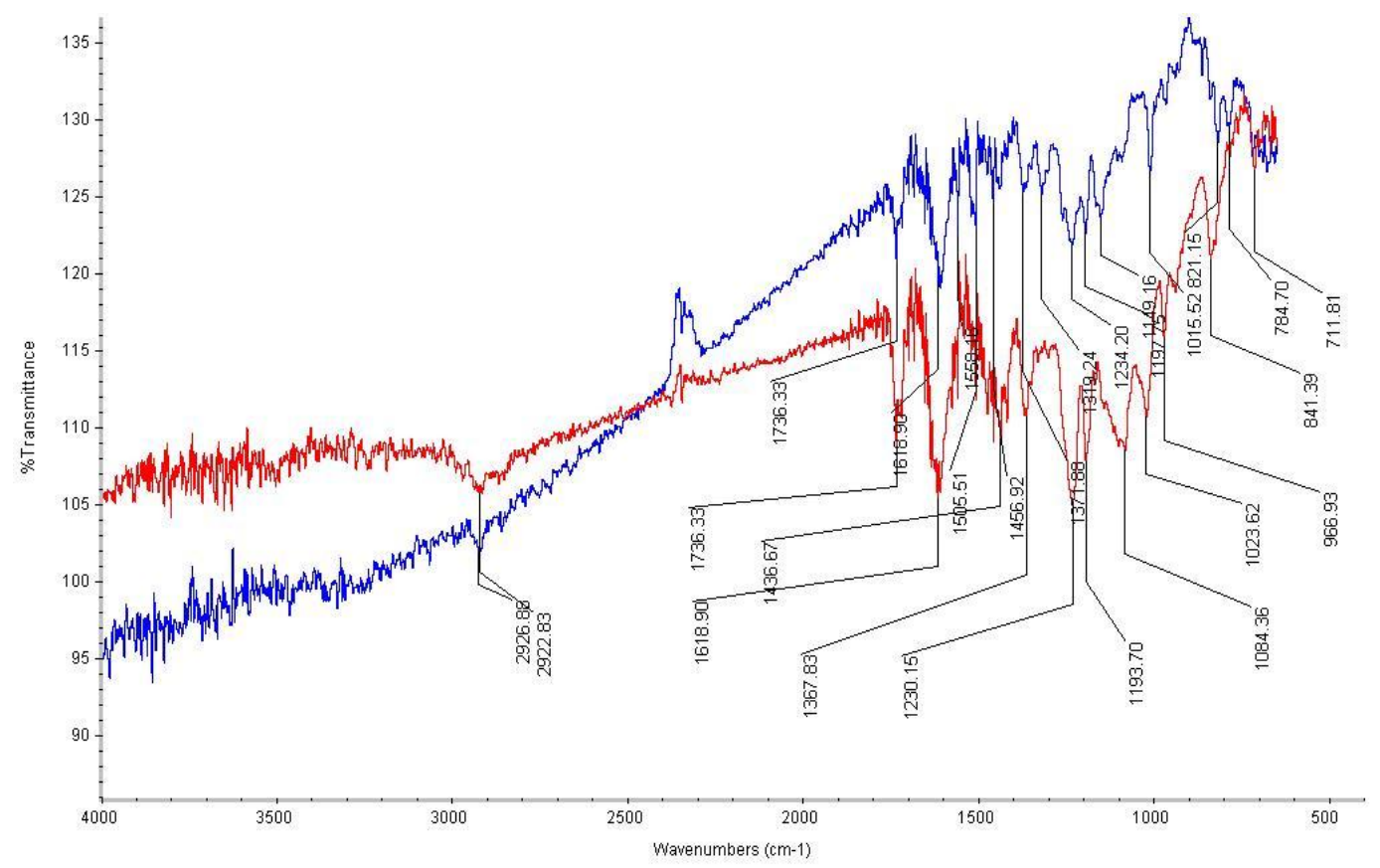

Figure 29: FT-IR spectra of the HME and physical mixture of 5\% by weight of quercetin in Soluplus ${ }^{\circledR}$ (Blue: physical mixture and red: HME mixture)

The spectra above show a few similarities in terms of the peak transmittance values. The broad peak at $2926 / \mathrm{cm}$ and the sharp peaks at $1735 / \mathrm{cm}$ and $1618 / \mathrm{cm}$ are seen in both spectra. The sharp peak obtained at $1015 / \mathrm{cm}$ in the physical mixture is not seen in the HME mixture. In the physical mixture, another medium peak appears at $1235 / \mathrm{cm}$ and such peaks are not observed in the HME mixture. Thus, the spectra of the HME mixture was matched with that of Soluplus ${ }^{\circledR}$ blank. This is shown in the following figure: 


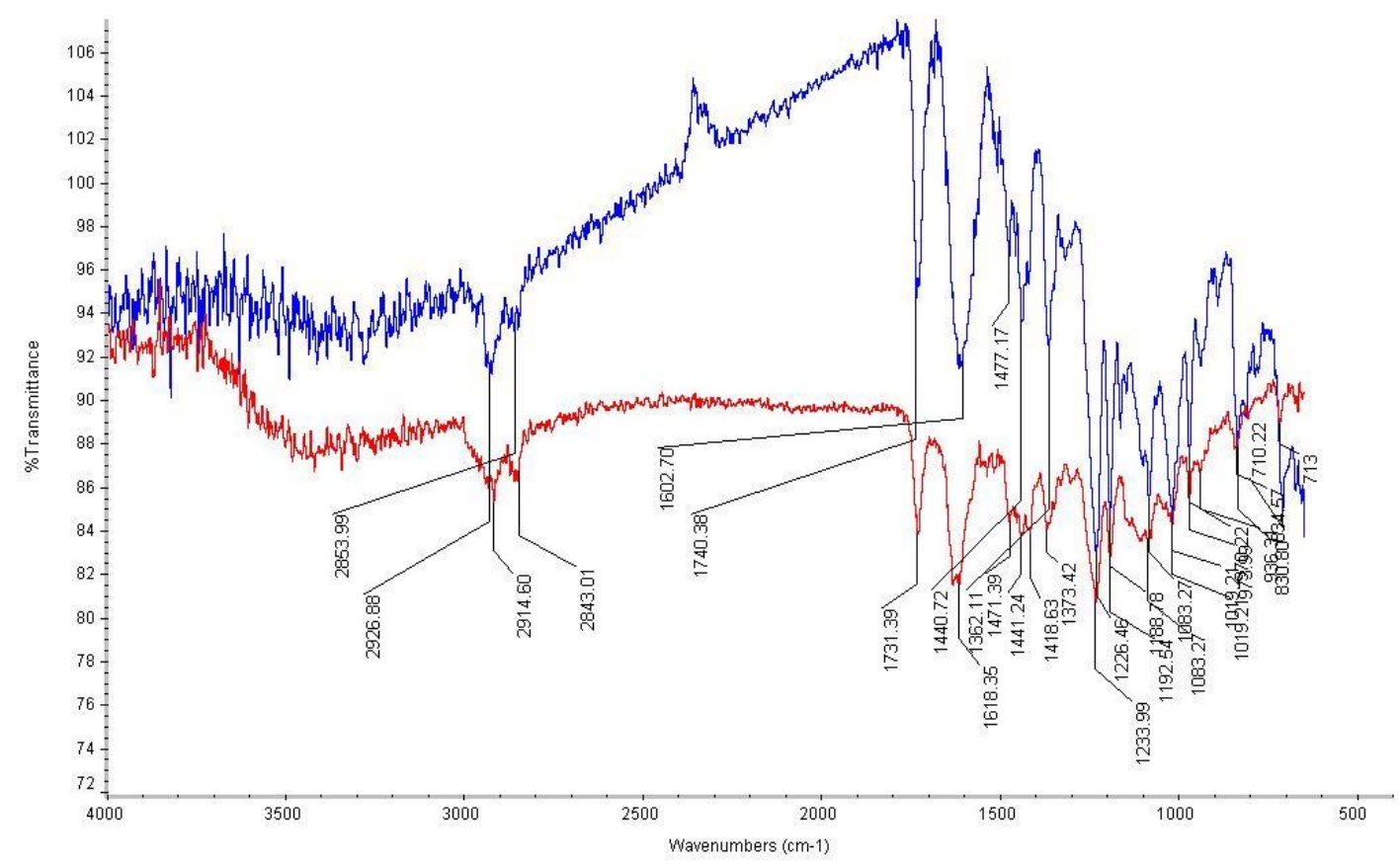

Figure 30: FT-IR spectra of quercetin HME and Soluplus ${ }^{\circledR}$ blank (Blue: quercetin $5 \%$ by weight in Soluplus® and red: Soluplus® blank)

The above spectra seem to fit into each other better than the spectra seen in the previous figure. The characteristic ketone wavelengths are observed in both spectra with the peaks between $1750 / \mathrm{cm}$ and $1600 / \mathrm{cm}$. There is a slight shifting in the peaks of the HME mixture towards a higher wavelength as compared to Soluplus ${ }^{\circledR}$. These shifts are also seen in the peaks observed in the HME mixture as compared to the peaks in Soluplus ${ }^{\circledR}$ in the wavelengths between $1500 / \mathrm{cm}$ and $1000 / \mathrm{cm}$. Lastly, the sharp peaks seen at wavelengths over $900 / \mathrm{cm}$ in Soluplus ${ }^{\circledR}$ are reduced to medium sized peaks in the HME mixture.

In conclusion, the FT-IR spectroscopy of the pure compounds and the hot-melt extruded mixtures of the polyphenols in Soluplus ${ }^{\circledR}$ have revealed that the chemical 
functionality of the polyphenols has undergone a change after the extrusion process with the polymer.

Resveratrol shows functional group properties similar to Soluplus ${ }^{\circledR}$ wherein, the spectra revealed nearly superimposable peaks for the melt extruded mixtures and the polymer. In the case of quercetin, a slight shift occurs in the peaks towards a higher wavelength as compared to the peaks of transmittance of the polymer. However, some of the functional group characteristics of quercetin remain similar even after hot-melt extrusion as opposed to resveratrol as can be seen from the peaks obtained due to the ketone group being retained at the same wavelength in quercetin after extrusion. The shift in the peaks may be due to the influence of the functional groups in the polymer which now lie in close proximity to the functional groups of quercetin after extrusion. Often, the shift in the $\mathrm{C}=\mathrm{O}$ stretching occurs due to conjugation by adjacent aromatic groups. This may be the case with quercetin after extrusion with Soluplus®. The shift in the peaks obtained due to the aromatic mononuclear and polynuclear groups between $1400 / \mathrm{cm}$ to $1000 / \mathrm{cm}$ are also attributed to the effects of the functional groups of the polymer on the bending of the $\mathrm{C}-\mathrm{H}$ within the ring for quercetin after extrusion. Lastly, the FT-IR spectroscopy assisted in observing the consistency of the extrusion process which was seen in the similar spectra obtained for the HME mixtures of both polyphenols (51). 
Study 6

A study of the stability, thermal properties and accelerated thermal degradation of resveratrol and quercetin using differential scanning calorimetry (DSC)

Results

The DSC analysis was designed in order to assist in studying the pre-formulation behavior of the polyphenols and Soluplus ${ }^{\circledR}$ when heated to a temperature less than the melting point of the polyphenols and additionally, to test the physical form (amorphous nature/absence of crystallinity) and determine the uniformity of the hotmelt extruded mixtures of the polyphenols in Soluplus ${ }^{\circledR}$. The first phase of this study involved the thermal study of the physical mixtures of the polyphenol in the polymer. The thermograms obtained as a result have been described below. The following figure shows the thermograms of the physical mixtures of resveratrol and Soluplus®.

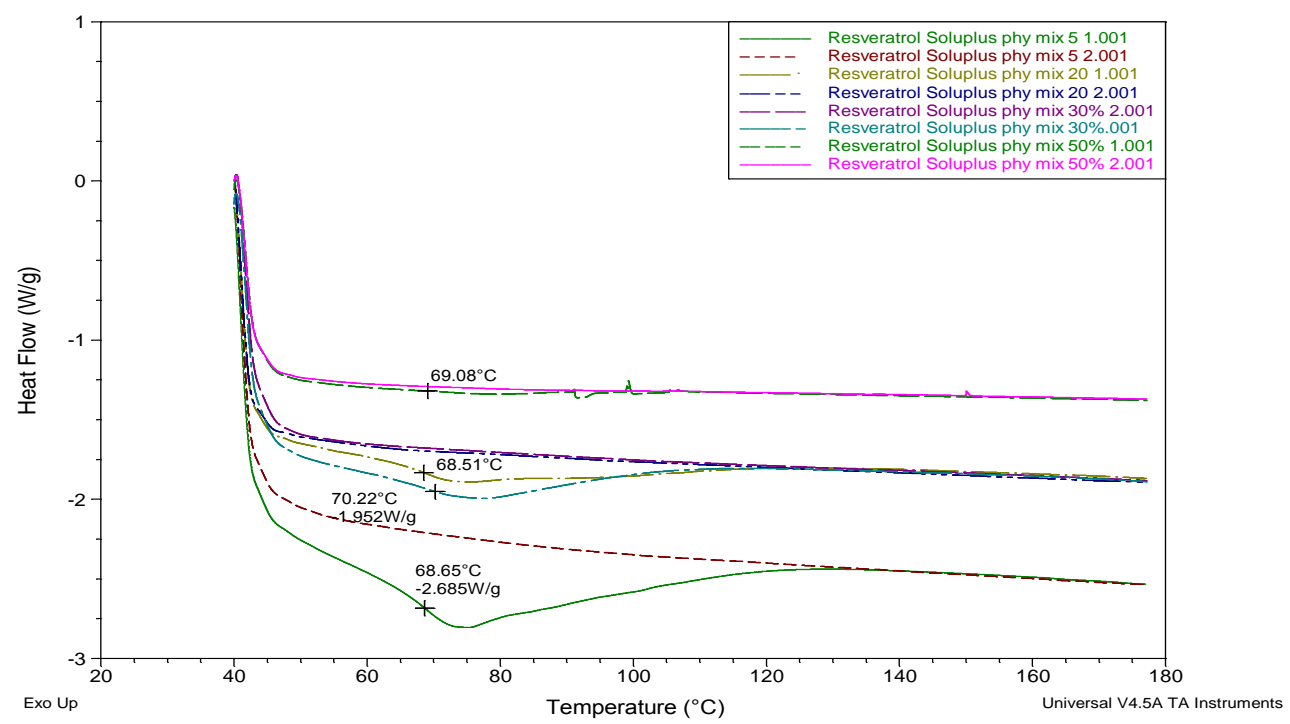

Figure 31: DSC thermograms of the physical mixtures of resveratrol in Soluplus ${ }^{\circledR}$. 
In the figure 31 above, the thermograms obtained for the different physical mixtures of resveratrol in Soluplus ${ }^{\circledR}$ can be observed. These runs were performed at temperatures lower than the melting point in order to determine and perhaps predict the behavior of resveratrol and Soluplus® under hot-melt extrusion. These mixtures containing 5\%, $20 \%, 30 \%$ and $50 \%$ of resveratrol have nearly the same glass transition temperatures which are similar to the glass transition temperature of Soluplus ${ }^{\circledR}\left(\sim 70^{\circ} \mathrm{C}\right)$. Thus, the glass transition temperature of the mixture containing 5\% resveratrol by weight is $68.65^{\circ} \mathrm{C}$; the mixture containing $20 \%$ resveratrol by weight has a glass transition temperature of $68.51{ }^{\circ} \mathrm{C}$ while that of the mixture containing $30 \%$ resveratrol by weight is $70.22^{\circ} \mathrm{C}$. The mixture containing $50 \%$ resveratrol by weight has a glass transition temperature around $69.08^{\circ} \mathrm{C}$. It can be observed that not much change has occurred in the glass transition temperature of Soluplus ${ }^{\circledR}$ when mixed with different weight ratios of resveratrol.

The figure 32 below shows the thermogram obtained for resveratrol alone. 


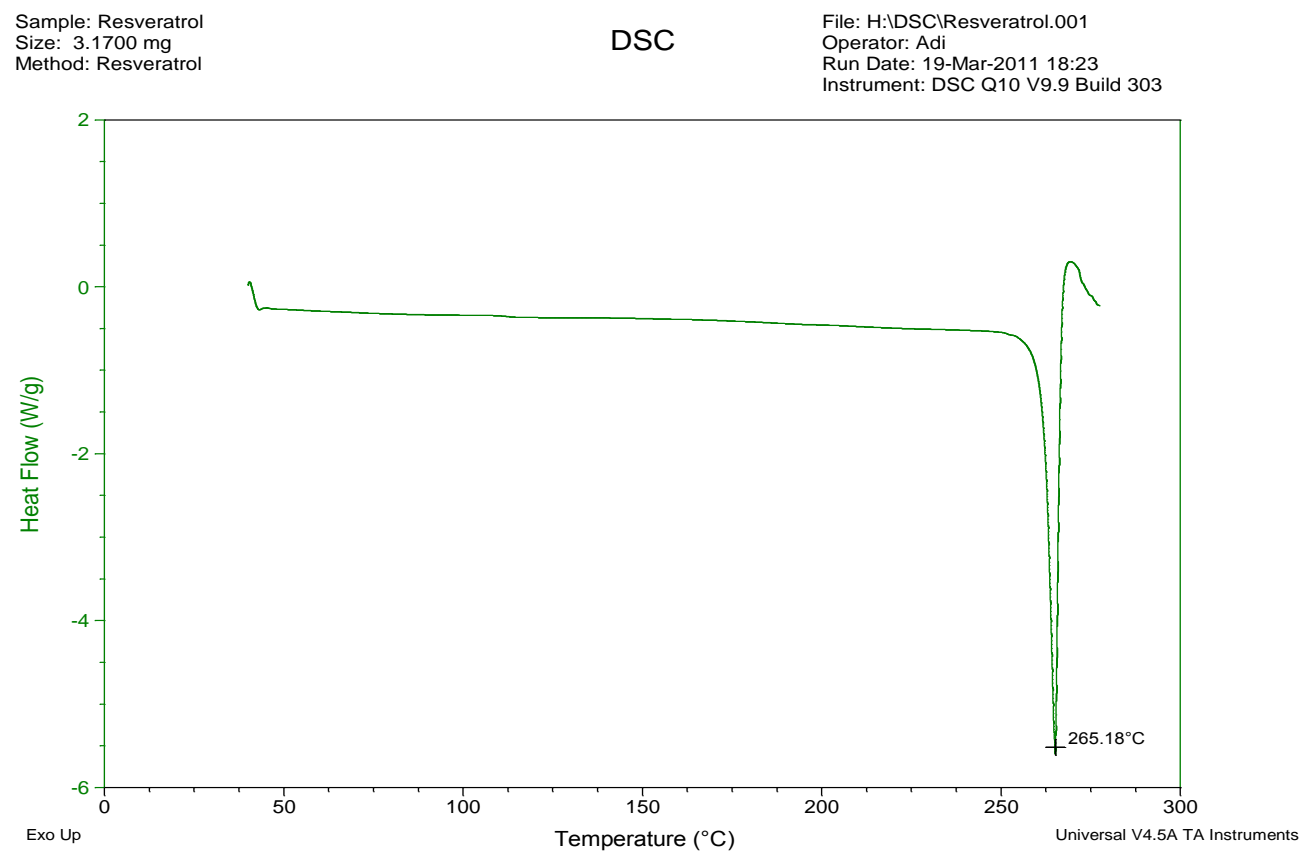

Figure 32: DSC thermogram of resveratrol

The figure 32 above shows the thermogram of resveratrol. As observed in the figure 32, the sharp peak is the melting point of resveratrol. This is observed as $265.18^{\circ} \mathrm{C}$ while the literature value is listed as $254^{\circ} \mathrm{C}$. 


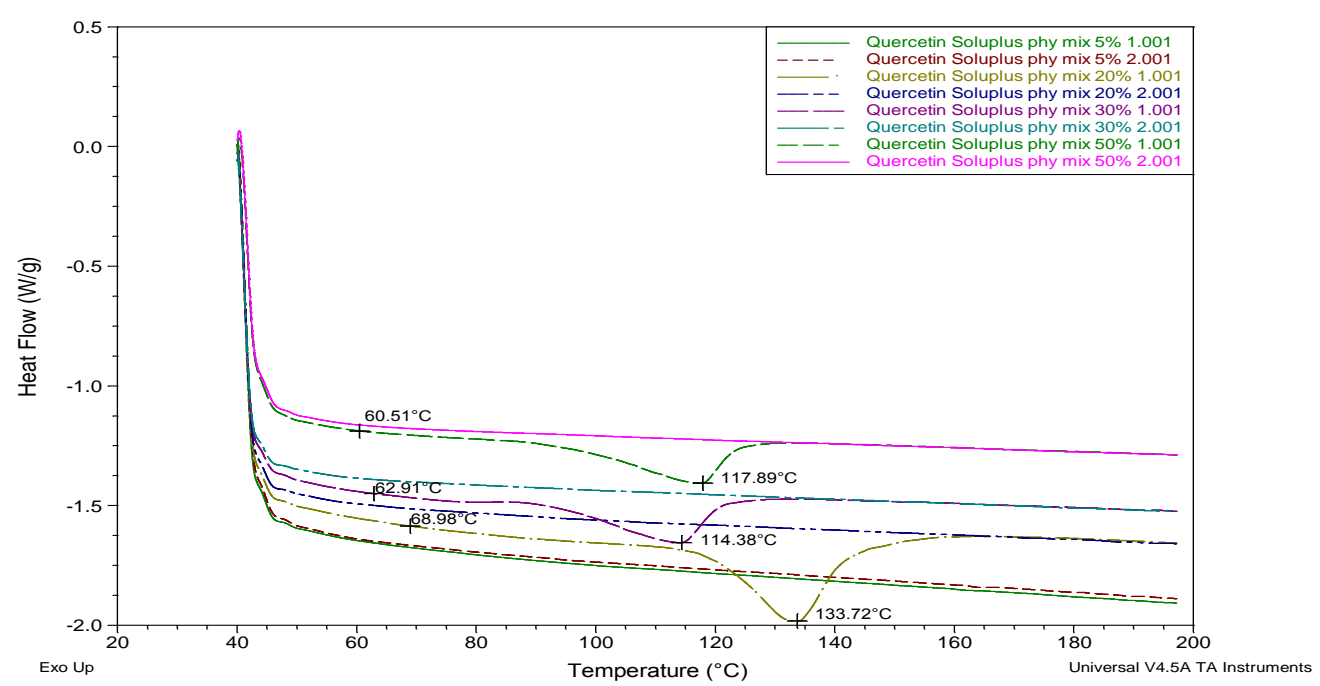

Figure 33: DSC thermograms of the physical mixtures of Quercetin in Soluplus ${ }^{\circ}$

The thermograms of various concentrations of quercetin by weight in Soluplus ${ }^{\circledR}$ were used as physical mixtures ranging from $5 \%$ to $50 \%$ as shown in figure 33 . The runs were conducted such that the first run was followed by immediate cooling of the sample, and a subsequent run. These can be observed from the pair of thermograms obtained for each sample as also carried out previously for resveratrol and Soluplus® physical mixtures and shown in figure 31.

From the thermograms, it is observed that the physical mixtures having quercetin up to an extent of $20 \%, 30 \%$ and $50 \%$ by weight show a glass transition within the sample in the temperature range of $115^{\circ} \mathrm{C}$ to $133^{\circ} \mathrm{C}$. These are characteristic of quercetin and are also observed in a pure sample of quercetin. This can be seen in a separate thermogram of a sample of quercetin shown in figure 34. For comparison, the thermogram of Soluplus ${ }^{\circledR}$ is shown in figure 35 below which shows a glass transition 
at close to $70^{\circ} \mathrm{C}$ and has no other glass transition phases. An interesting characteristic of these thermograms is the reduction in the glass transition temperature value as the amount of quercetin in the sample increases from $20 \%$ to $50 \%$ by weight. Thus, the mixture containing 50\% quercetin shows a change in the curve (glass transition) at $115^{\circ} \mathrm{C}$. This increases to $118^{\circ} \mathrm{C}$ in the mixture containing $30 \%$ by weight of quercetin and further increases to $133^{\circ} \mathrm{C}$ in the mixture containing $20 \%$ by weight of quercetin. The mixture containing $5 \%$ by weight of quercetin shows a glass transition temperature of close to $70^{\circ} \mathrm{C}$ which is not very clearly visible in the endotherm but occurs as a slight change in the curve. In the physical mixtures of quercetin and Soluplus $®$, a reduction in the glass transition temperatures of Soluplus ${ }^{\circledR}$ is observed as the concentration of quercetin in the sample increases. In the physical mixture containing $20 \%$ by weight of quercetin and Soluplus®, the glass transition temperature is observed as $68.98^{\circ} \mathrm{C}$. Further, the $\mathrm{Tg}$ (glass transition temperature) of the physical mixtures containing $30 \%$ and $50 \%$ by weight of quercetin and Soluplus ${ }^{\circledR}$ are $62.91{ }^{\circ} \mathrm{C}$ and $60.51^{\circ} \mathrm{C}$ respectively.

From figure 33 , it is observed that an increase in the concentration of quercetin in the mixture of quercetin and Soluplus ${ }^{\circledR}$ has caused a depression in the glass transition temperature. Thus, quercetin has an apparent plasticization effect on Soluplus ${ }^{\circledR}$ causing reduction in the glass transition temperature. In the case of resveratrol, there is no significant change in the glass transition temperature as the concentration of resveratrol increases in the mixture. Summarily, quercetin may have a certain plasticization effect on the polymer Soluplus ${ }^{\circledR}$ while resveratrol produces no such effect. The following thermogram in figure 34 is the thermogram of quercetin alone. 


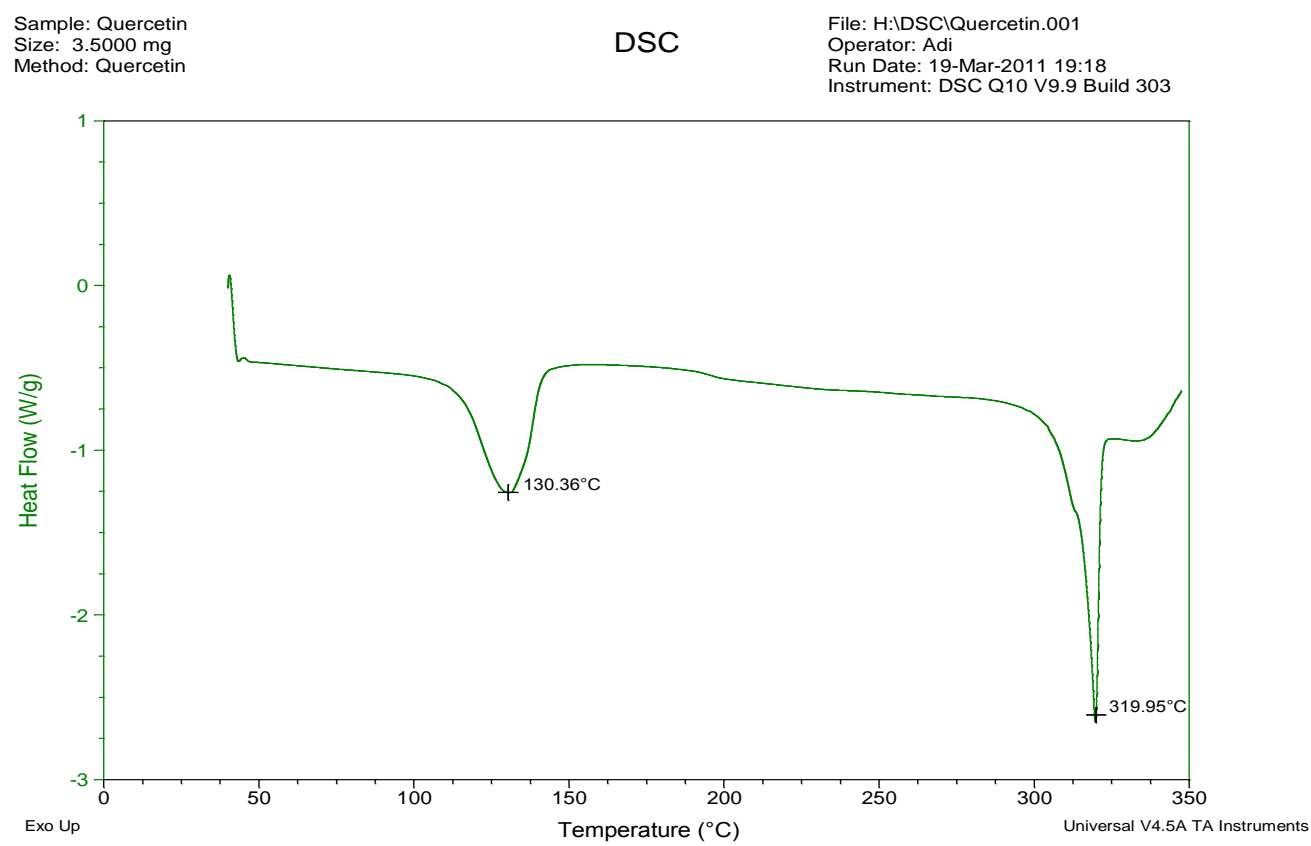

Figure 34: DSC thermogram of quercetin

Figure 34 shows the DSC thermogram of quercetin. As can be seen from the thermogram, the melting point or melting temperature $(\mathrm{tm})$ is obtained as a sharp peak. This is observed at $319.95^{\circ} \mathrm{C}$ in the sample while the tm has been recorded as $316^{\circ} \mathrm{C}$ in the literature. A glass transition is observed in quercetin at $130.36^{\circ} \mathrm{C}$ which was also seen in the thermograms in figure 33.

The following thermogram was obtained on the DSC analysis of a sample of Soluplus ${ }^{\circledR}$. 


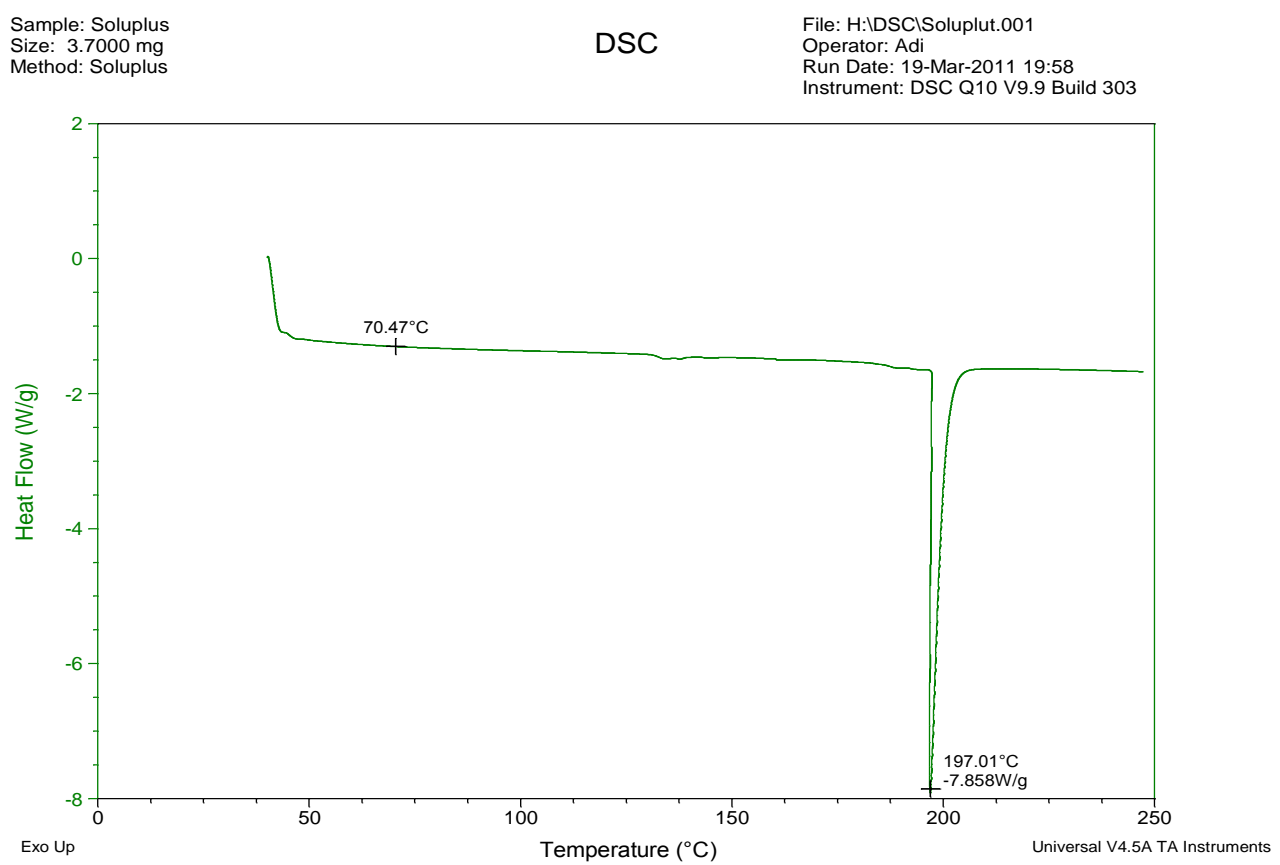

Figure 35: DSC thermogram of Soluplus $®$.

The figure 35 above shows the thermogram of Soluplus ${ }^{\circledR}$. The typical features of this thermogram are the glass transition temperature at $70.47^{\circ} \mathrm{C}$ that occurs as a slight change in the curve and the sharp melting peak obtained at $\sim 197^{\circ} \mathrm{C}$. No other glass transition phases are observed for Soluplus ${ }^{\circledR}$ as seen in figure 35.

Phase two of the DSC test involved the analysis of the HME mixtures of resveratrol and quercetin in Soluplus ${ }^{\circledR}$ in order to determine their uniformity and detect any recrystallization of the constituents if present. Figure 36 below shows the thermograms obtained for the HME mixtures of resveratrol and Soluplus® along with the thermograms of their corresponding physical mixtures. 


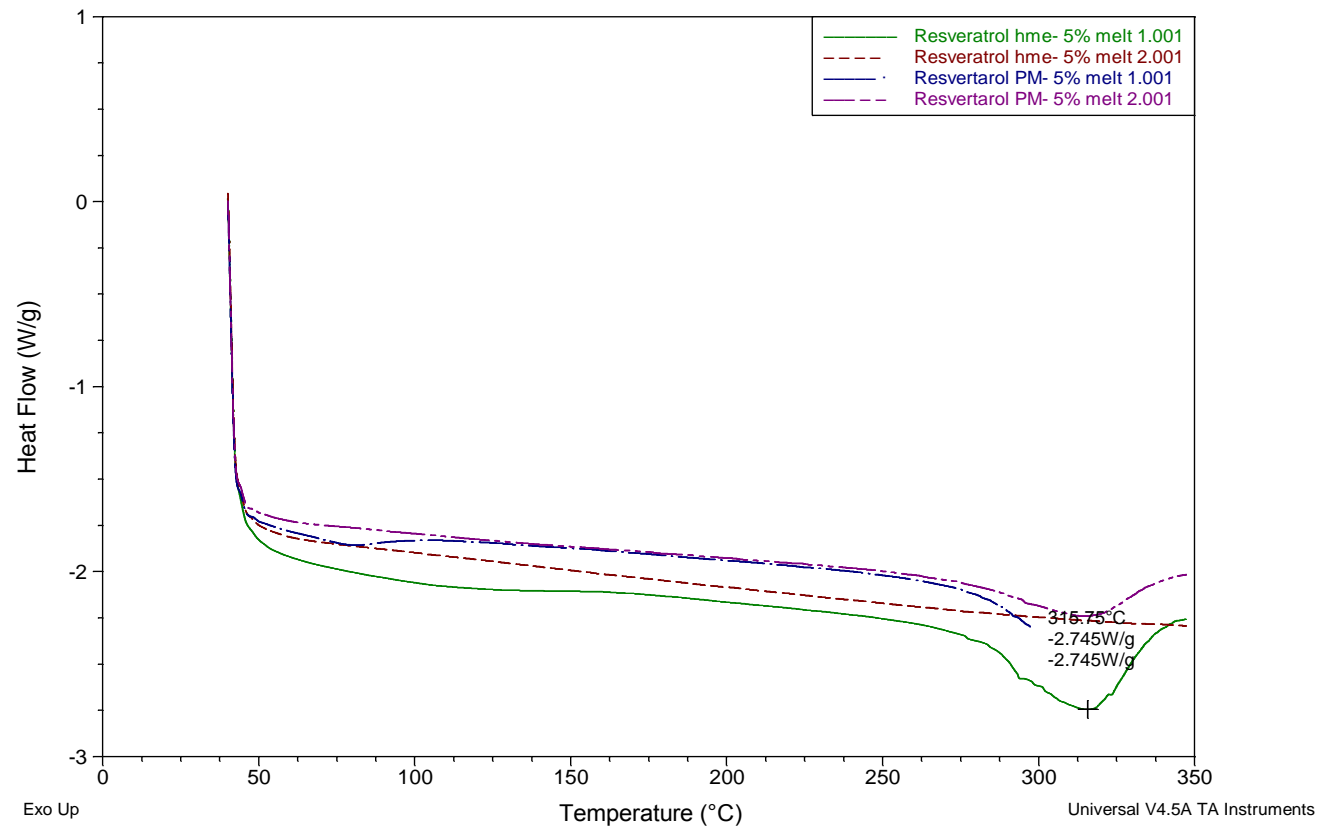

Figure 36: DSC thermograms of the hot-melt extruded and the physical mixture of $5 \%$ resveratrol by weight and Soluplus ${ }^{\circledR}$.

In the thermograms in figure 36 above, it is observed that, both the mixtures melt at close to $316^{\circ} \mathrm{C}$. The melting point of pure resveratrol is $254^{\circ} \mathrm{C}$. In the thermogram obtained for pure resveratrol, this melt occurred at close to $265^{\circ} \mathrm{C}$ as seen in figure 32.Thus, the mixtures melt at a significantly higher temperature than the pure compound. The second run carried out after cooling the sample at the end of the initial run revealed no sharp melting peaks or crystallinity. Glass transition is a characteristic feature of the amorphous regions within a polymer while a sharp melting peak or melting temperature is indicative of a crystalline substance. Thus, the product remained amorphous after extrusion, but melted at an extremely high temperature. A similar run was carried out for the mixtures containing $20 \%$ by 
weight of resveratrol and Soluplus®. The thermograms obtained have been shown in figure 37 below.

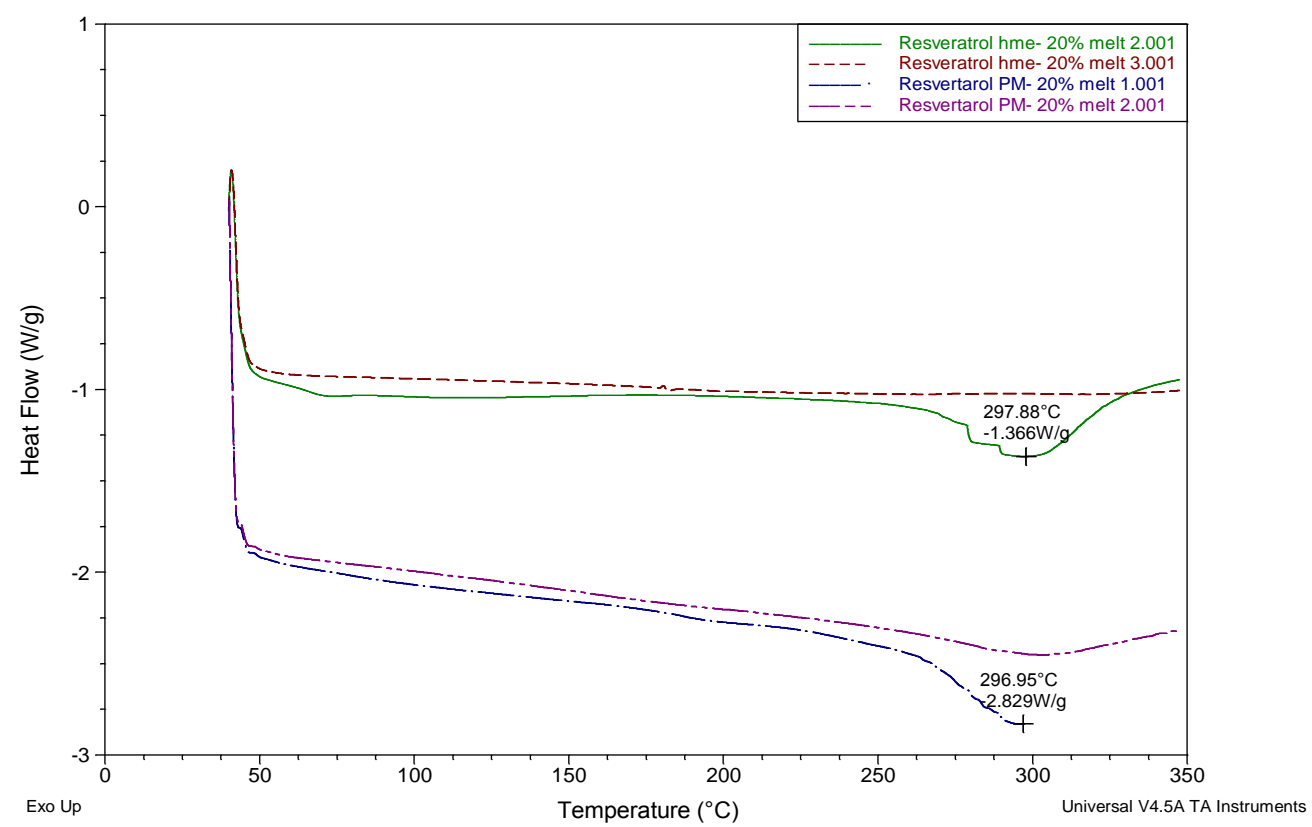

Figure 37: DSC thermograms of the hot-melt extruded and the physical mixture of $20 \%$ resveratrol by weight and Soluplus ${ }^{\circledR}$.

In the thermograms in figure 37 above, a pattern similar to the mixtures in containing 5\% resveratrol by weight are observed. However, the melting point of the mixtures containing $20 \%$ by weight of resveratrol is lower than the mixtures containing $5 \%$ by weight of resveratrol. The physical mixture and the HME mixture containing $20 \%$ resveratrol by weight melt at a temperature of about $297^{\circ} \mathrm{C}$. 
In figures 36 and 37, the first run for the physical mixture was performed up to a temperature of $300^{\circ} \mathrm{C}$. The mixtures of $5 \%$ resveratrol by weight and Soluplus ${ }^{\circledR}$ (HME and physical mixtures) melted at $315^{\circ} \mathrm{C}$ and at close to $297^{\circ} \mathrm{C}$ in the physical mixture containing $20 \%$ resveratrol by weight. The final product obtained after hot-melt extrusion, thus, had a significantly higher melting point than pure polyphenolic resveratrol. This was confirmed when the HME mixtures containing the two weight fractions of resveratrol (5\% and $20 \%$ by weight of resveratrol) melted at temperatures similar to their corresponding physical mixtures.

The following figures 38 and 39 show the thermograms obtained for the HME and the physical mixtures of quercetin and Soluplus ${ }^{\circledR}$. 


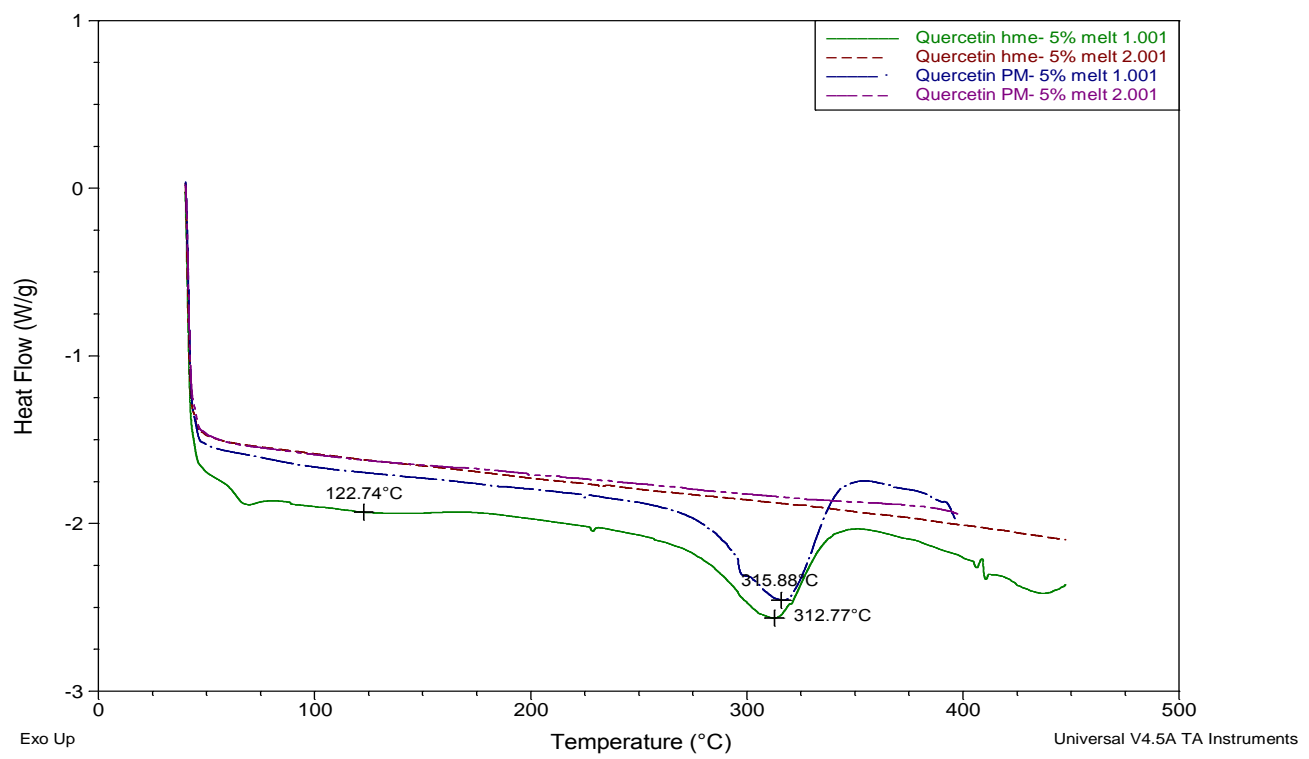

Figure 38: DSC thermograms of the hot-melt extruded and the physical mixture of $5 \%$ quercetin by weight and Soluplus®.

In figure 38 above, the thermograms for the physical mixture and the HME mixture containing $5 \%$ quercetin by weight and Soluplus ${ }^{\circledR}$ are observed. As compared to the thermogram obtained in figure 34 , the mixtures are seen to melt at a temperature of close to $315^{\circ} \mathrm{C}$. The melting point of pure quercetin is $316^{\circ} \mathrm{C}$ and the melting temperature obtained for the quercetin sample as seen in figure 34 is close to $320^{\circ} \mathrm{C}$. Thus, in this mixture there is no increase in the melting point as compared to pure quercetin as was observed with the mixtures containing $5 \%$ resveratrol by weight and Soluplus®. The melting point of the mixtures also does not appear like a sharp crystalline melting transition. Additionally, a glass transition phase in the HME and physical mixture is observed at close to $123^{\circ} \mathrm{C}$. This glass transition shows that the HME mixture of $5 \%$ by weight of quercetin and Soluplus ${ }^{\circledR}$ is amorphous. The figure 
39 shows the thermograms obtained for the HME and physical mixtures containing $20 \%$ quercetin by weight and Soluplus®.

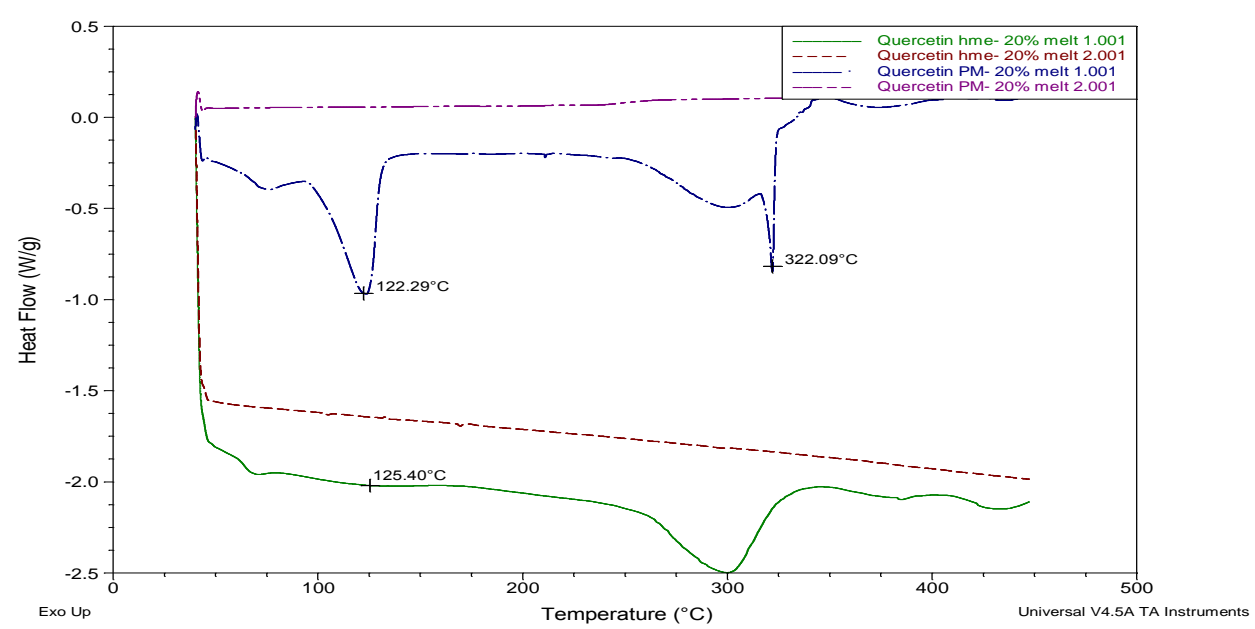

Figure 39: DSC thermograms of the hot-melt extruded and the physical mixture of $20 \%$ quercetin by weight and Soluplus ${ }^{\circledR}$.

In figure 39, the thermograms obtained for the HME and physical mixtures containing $20 \%$ quercetin by weight and Soluplus ${ }^{\circledR}$ have been shown. The glass transition temperatures are nearly similar and the melting temperatures for both mixtures are different. The $\operatorname{tg}$ of the HME mixture is $125.40^{\circ} \mathrm{C}$ while that of the physical mixture is $122.29^{\circ} \mathrm{C}$. In comparison with the HME mixture containing $5 \%$ by weight of quercetin and Soluplus®, the HME mixture containing $20 \%$ by weight of quercetin and Soluplus ${ }^{\circledR}$ shows a lower melting temperature while the physical mixture of $20 \%$ by weight of quercetin and Soluplus ${ }^{\circledR}$ shows a melting temperature of $322.09^{\circ} \mathrm{C}$.

In conclusion, the pre-formulation studies revealed that the in the physical mixtures of resveratrol, the glass transition phase for Soluplus ${ }^{\circledR}$ was observed whose temperature 
range was remained nearly the same as the concentration of resveratrol was increased to about $50 \%$ by weight.

The physical mixtures and HME mixtures of quercetin displayed two glass transition phases (one for Soluplus ${ }^{\circledR}$ and the other for quercetin) and the increase in the amount of quercetin had an effect of plasticization or the reduction in the glass transition temperature of Soluplus ${ }^{\circledR}$.

The DSC runs of the HME mixtures of resveratrol showed that there was a significant increase in the melting point of the final product as compared to the pure sample of polyphenolic resveratrol. However, as the concentration of resveratrol increased from $5 \%$ to $20 \%$ by weight, the melting point of mixtures was reduced. The DSC runs also showed that there was no sharp melting peaks characteristic of crystalline substances which might indicate the amorphous nature of these mixtures after extrusion. Similarly, the DSC runs of the HME mixtures of quercetin showed no sharp melting peaks and displayed a glass transition temperature above $120^{\circ} \mathrm{C}$. Glass transition temperatures occur in the amorphous part of a polymer molecule wherein a phase transition occurs from the glassy to the rubbery state. Thus, the absence of sharp crystalline melting peaks and the presence of a glass transition phase might show that the HME mixture of quercetin and Soluplus ${ }^{\circledR}$ remains amorphous after extrusion. In the case of the HME mixtures of quercetin, again different melting temperatures were observed for the HME and physical mixture containing $5 \%$ by weight of quercetin and the HME and physical mixture containing $20 \%$ by weight of quercetin. 
Study 7

A study of the properties of tablets produced from resveratrol and quercetin

Results

The hot-melt extruded and the physical mixtures of the polyphenols in Soluplus ${ }^{\circledR}$ were formulated into tablets weighing approximately $200 \mathrm{mg}$ each. These tablets were subjected to the tests described in the methodology earlier. The data obtained as a result was used for the comparison of the properties of these mixtures upon formulation as tablets. The figures below depict the tablets formulated from a few of the mixtures.

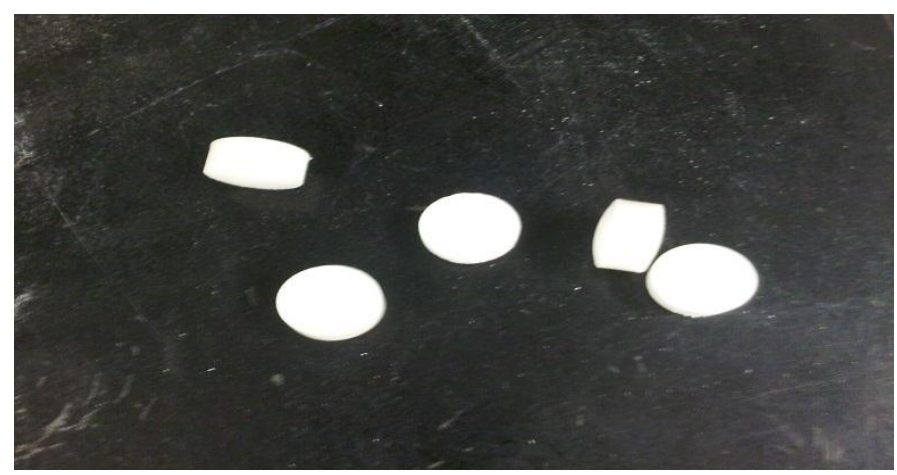

Figure 40: Tablets of HME mixtures containing $2 \%$ resveratrol by weight and Soluplus ${ }^{\circledR}$. 


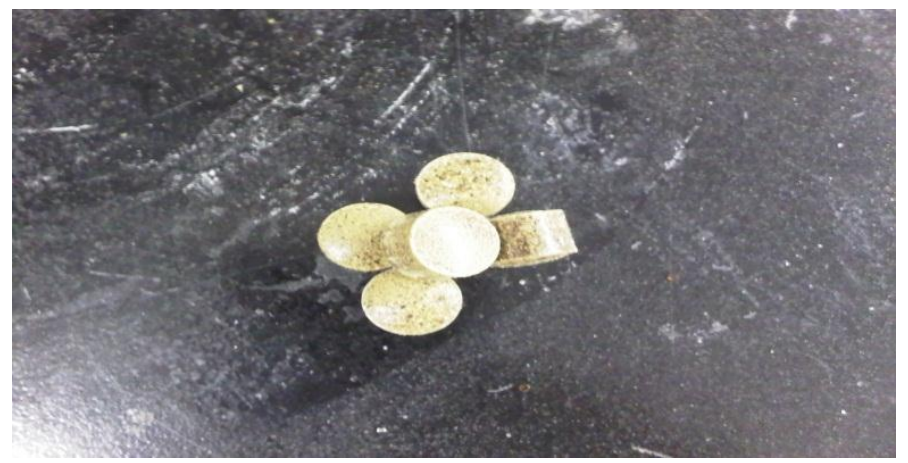

Figure 41: Tablets of HME mixtures containing 5\% quercetin by weight and Soluplus®.

The following tables describe the data obtained for the three types of characterization tests carried out on the tablets. 


\begin{tabular}{|c|c|c|c|c|c|}
\hline \multicolumn{6}{|c|}{ Hardness test for mixtures containing resveratrol } \\
\hline \multicolumn{3}{|c|}{ Physical mixture } & \multicolumn{3}{|c|}{ Hot-melt extruded mixture } \\
\hline $2 \%$ & $5 \%$ & $20 \%$ & $2 \%$ & $5 \%$ & $20 \%$ \\
\hline resveratrol & resveratrol & resveratrol & resveratrol & resveratrol & resveratrol \\
\hline by weight & by weight & by weight & by weight & by weight & by weight \\
\hline + Soluplus & +Soluplus & +Soluplus & +Soluplus & + Soluplus & + Soluplus \\
\hline \multicolumn{6}{|c|}{ Hardness in kilograms } \\
\hline 3 & 4.3 & 1.8 & 5.0 & 6.5 & 5.1 \\
\hline 3.5 & 4.4 & 2.0 & 5.2 & 6.3 & 4.8 \\
\hline 3.5 & 4.2 & 2.0 & 5.3 & 6.5 & 4.8 \\
\hline 3.4 & 4.4 & 2.1 & 5.1 & 6.5 & 4.9 \\
\hline 3.5 & 4.4 & 1.9 & 5.1 & 6.4 & 5 \\
\hline Average & Average & Average & Average & Average & Average \\
\hline hardness $=$ & hardness $=$ & hardness $=$ & hardness $=$ & hardness = & hardness $=$ \\
\hline $3.38 \mathrm{~kg}$ & $4.34 \mathrm{~kg}$ & $1.96 \mathrm{~kg}$ & $5.14 \mathrm{~kg}$ & $6.44 \mathrm{~kg}$ & $4.92 \mathrm{~kg}$ \\
\hline
\end{tabular}

Table 7: The average hardness of the tablets of the HME and corresponding physical mixtures of resveratrol with Soluplus ${ }^{\circledR}$ in kilograms

In table 7 above, the data obtained for the hardness test performed on the tablets produced from the HME and physical mixtures containing resveratrol have been shown. The test was performed on the Erweka hardness tester which measures the weight required in kilograms to break a tablet as its hardness parameter. The data shows that the average hardness of the tablets produced from the HME mixtures is 
higher than those produced from the physical mixtures of resveratrol and Soluplus ${ }^{\circledR}$. In a given set of mixtures, the hardness of the tablets increases when the concentration of resveratrol increases from $2 \%$ to $5 \%$ by weight in the mixture. When the concentration of resveratrol reaches $20 \%$ by weight, the hardness again decreases. The physical mixtures containing $20 \%$ resveratrol by weight and Soluplus ${ }^{\circledR}$ were very brittle and broke upon slight pressure as can be seen from the low values of hardness. Thus, tablets produced from the HME mixtures were harder than the tablets produced by the physical mixture. The following table shows the data obtained for the hardness test performed on the tablets produced from the HME and the physical mixtures of quercetin and Soluplus ${ }^{\circledR}$. 


\begin{tabular}{|c|c|c|c|}
\hline \multicolumn{4}{|c|}{ Hardness test for mixtures containing quercetin } \\
\hline \multicolumn{2}{|c|}{ Physical mixture } & \multicolumn{2}{|c|}{ Hot-melt extruded } \\
\hline $\begin{array}{l}5 \% \text { quercetin by } \\
\text { weight } \\
+ \text { Soluplus }{ }^{\circledR}\end{array}$ & $\begin{array}{l}20 \% \text { quercetin by } \\
\text { weight } \\
+ \text { Soluplus }{ }^{\circledR}\end{array}$ & $\begin{array}{l}5 \% \text { quercetin by } \\
\text { weight } \\
\text { +Soluplus }{ }^{\circledR}\end{array}$ & $\begin{array}{l}20 \% \text { quercetin by } \\
\text { weight } \\
\text { +Soluplus }{ }^{\circledR}\end{array}$ \\
\hline \multicolumn{4}{|c|}{ Hardness in kilograms } \\
\hline 5.1 & 0 & 7.0 & 6.2 \\
\hline 4.9 & 0 & 7.3 & 6.2 \\
\hline 5.0 & 0 & 7.2 & 6.0 \\
\hline 5.0 & 0 & 7.3 & 6.1 \\
\hline 5.0 & 0 & 7.0 & 6.2 \\
\hline $\begin{array}{l}\text { Average hardness }= \\
5 \mathrm{~kg}\end{array}$ & $\begin{array}{l}\text { Average hardness }= \\
0 \mathrm{~kg}\end{array}$ & $\begin{array}{l}\text { Average hardness= } \\
7.16 \mathrm{~kg}\end{array}$ & $\begin{array}{l}\text { Average hardness }= \\
6.14 \mathrm{~kg}\end{array}$ \\
\hline
\end{tabular}

Table 8: Data obtained for the hardness test performed on the tablets produced from the physical mixtures and the hot-melt extruded mixtures of quercetin and Soluplus $®$.

Table 8 above shows the data obtained for the hardness test performed on the physical mixtures and the HME mixtures of quercetin and Soluplus ${ }^{\circledR}$. The test was performed using the same method and instrument employed for the mixtures of resveratrol and Soluplus®. The striking feature of this table is the absence of data for the physical mixture containing $20 \%$ quercetin by weight and Soluplus ${ }^{\circledR}$. This is because the formulation of this mixture into tablets failed completely and only masses of compact mixtures that disintegrated upon application of light pressure were obtained on pressing within the tablet press. The data also shows that tablets produced from the 
physical mixtures containing quercetin and Soluplus ${ }^{\circledR}$ were harder than the tablets produced by the physical mixture of resveratrol and Soluplus ${ }^{\circledR}$. Another feature of the data is the increased hardness of the HME mixtures containing quercetin and Soluplus ${ }^{\circledR}$ as compared to the corresponding physical mixtures. The average hardness for the tablets produced from these mixtures is above $6 \mathrm{~kg}$. Thus, harder tablets were produced from the mixtures containing quercetin as compared to the mixtures containing resveratrol and the physical mixture containing $20 \%$ quercetin by weight and Soluplus ${ }^{\circledR}$ could not be successfully formulated into tablets.

The following table shows the data obtained for the friability test performed on the tablets produced. 


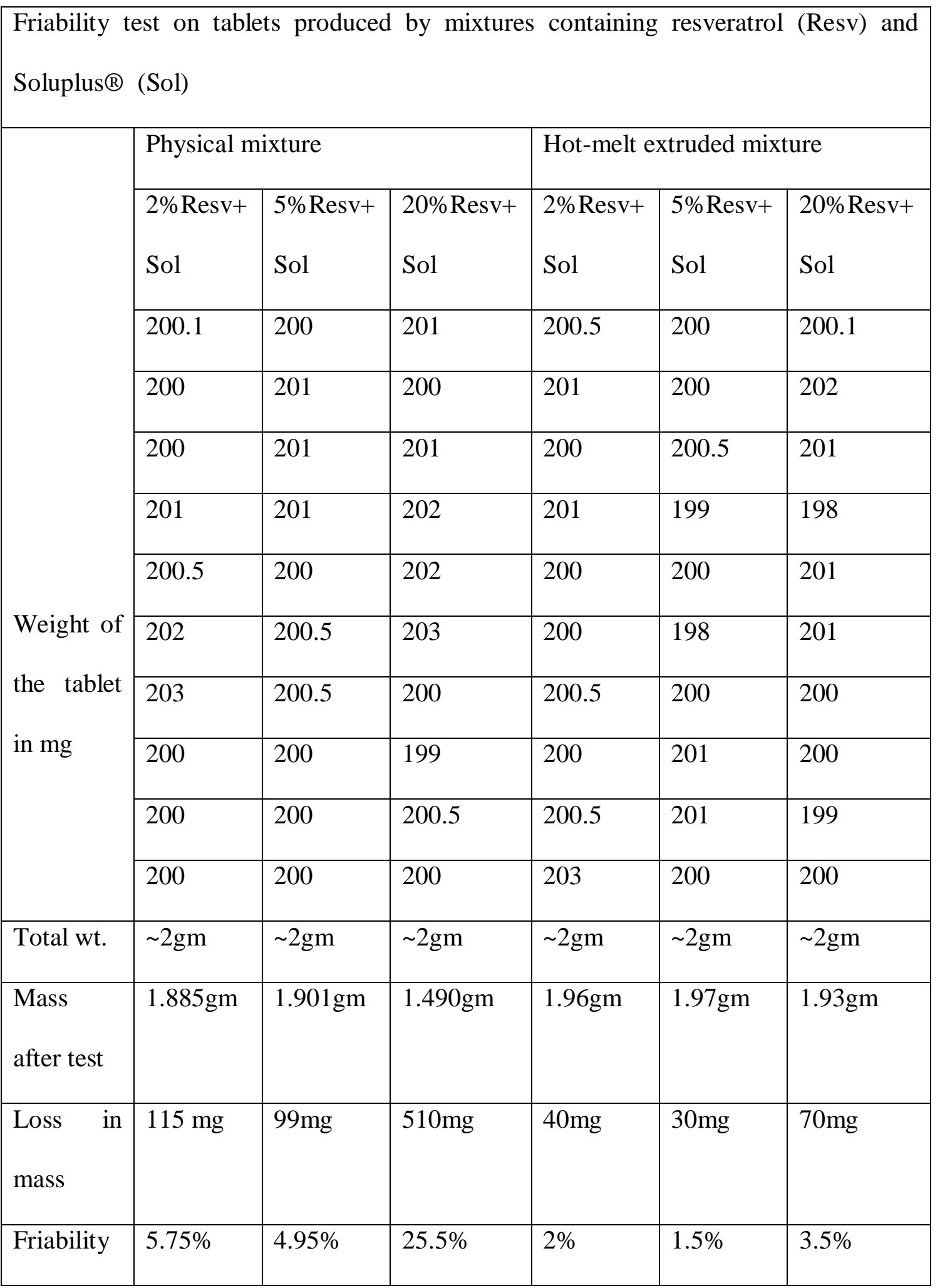

Table 9: Data showing the friability in percentage for the tablets produced from the physical mixture and the HME mixture containing resveratrol and Soluplus®. 
In table 9 above, the percent friability of the tablets produced by the physical mixture and the hot-melt extruded mixture containing resveratrol and quercetin have been shown.

From the table, it is observed that the tablets produced from the physical mixture are more friable as compared to the tablets produced from the HME mixtures. The friability values obtained for the physical mixtures containing resveratrol and Soluplus ${ }^{\circledR}$ are relatively higher than the tablets produced from the HME mixtures of the same. The tablets containing $5 \%$ resveratrol by weight have the lowest friability while the tablets produced from the physical mixture containing $20 \%$ resveratrol by weight have the highest friability. The values of friability assist in determining the loss in mass or damage the tablets may undergo during the process of packaging, storage and transportation. Additionally, they are indicators of the hardness of a tablet in a certain way. Thus, predictably, as the tablets produced from the physical mixture containing $20 \%$ by weight of resveratrol had the lowest hardness values, they are observed to be the most friable from the data above. The following table shows the data obtained for the friability test conducted on the tablets produced from the mixtures of quercetin and Soluplus ${ }^{\circledR}$. 


\begin{tabular}{|c|c|c|c|c|}
\hline \multicolumn{5}{|c|}{$\begin{array}{l}\text { Friability test on the tablets pr } \\
\text { Soluplus }{ }^{\circledR}(\text { Sol). }\end{array}$} \\
\hline \multirow{12}{*}{$\begin{array}{l}\text { Weight } \\
\text { of the } \\
\text { tablet in } \mathrm{mg}\end{array}$} & \multicolumn{2}{|l|}{ Physical mixture } & \multicolumn{2}{|c|}{ Hot-melt extruded mixture } \\
\hline & $\begin{array}{l}\text { 5\% Quer + } \\
\text { Sol }\end{array}$ & $\begin{array}{l}20 \% \text { Quer }+ \\
\text { Sol }\end{array}$ & $5 \%$ Quer + Sol & $\begin{array}{l}20 \% \text { Quer + } \\
\text { Sol }\end{array}$ \\
\hline & 200 & $\mathrm{n} / \mathrm{a}$ & 202 & 199 \\
\hline & 200.1 & $\mathrm{n} / \mathrm{a}$ & 201 & 200 \\
\hline & 200 & $\mathrm{n} / \mathrm{a}$ & 200 & 200 \\
\hline & 200 & $\mathrm{n} / \mathrm{a}$ & 202 & 200 \\
\hline & 200 & $\mathrm{n} / \mathrm{a}$ & 200.5 & 201 \\
\hline & 201 & $\mathrm{n} / \mathrm{a}$ & 202 & 200 \\
\hline & 198 & $\mathrm{n} / \mathrm{a}$ & 201 & 200 \\
\hline & 200 & $\mathrm{n} / \mathrm{a}$ & 200 & 201 \\
\hline & 200 & $\mathrm{n} / \mathrm{a}$ & 199 & 200 \\
\hline & 200.5 & $\mathrm{n} / \mathrm{a}$ & 200.5 & 200 \\
\hline Total weight & $\sim 2 \mathrm{gm}$ & $\mathrm{n} / \mathrm{a}$ & $\sim 2 \mathrm{gm}$ & $\sim 2 \mathrm{gm}$ \\
\hline Mass after test & $1.700 \mathrm{gm}$ & $\mathrm{n} / \mathrm{a}$ & $1.985 \mathrm{gm}$ & $1.972 \mathrm{gm}$ \\
\hline Loss in mass & $300 \mathrm{mg}$ & $\mathrm{n} / \mathrm{a}$ & $15 \mathrm{mg}$ & $28 \mathrm{mg}$ \\
\hline Friability & $15 \%$ & $\mathrm{n} / \mathrm{a}$ & $0.75 \%$ & $1.4 \%$ \\
\hline
\end{tabular}

Table 10: Data obtained for the friability test on the tablets produced from the physical mixtures and the hot-melt extruded mixtures of quercetin and Soluplus ${ }^{\circledR}$ 
Table 10 shows the data obtained for the friability test conducted on the physical mixtures and the hot-melt extruded mixtures of quercetin and Soluplus®. From the table, it is observed that the tablets produced by the physical mixture containing $5 \%$ quercetin by weight are the most friable with a value totaling $15 \%$. The friability of the tablets produced from the HME mixtures of quercetin and Soluplus $®$ are least friable as compared to the tablets produced from the physical mixture of quercetin and Soluplus ${ }^{\circledR}$ and the tablets produced from the physical mixtures and the HME mixtures of resveratrol and Soluplus®. Again, the table does not show any data for the tablets of the physical mixture containing $20 \%$ quercetin by weight and Soluplus ${ }^{\circledR}$ as the mixture could not be successfully formulated into tablets. Thus, the data shows that the tablets produced by the HME mixtures of quercetin are least friable and these are confirmed by their higher hardness values obtained from the hardness test.

The following table shows the data obtained from the disintegration test for the tablets produced from the physical mixture and the hot-melt extruded (HME) mixtures of resveratrol and quercetin. 


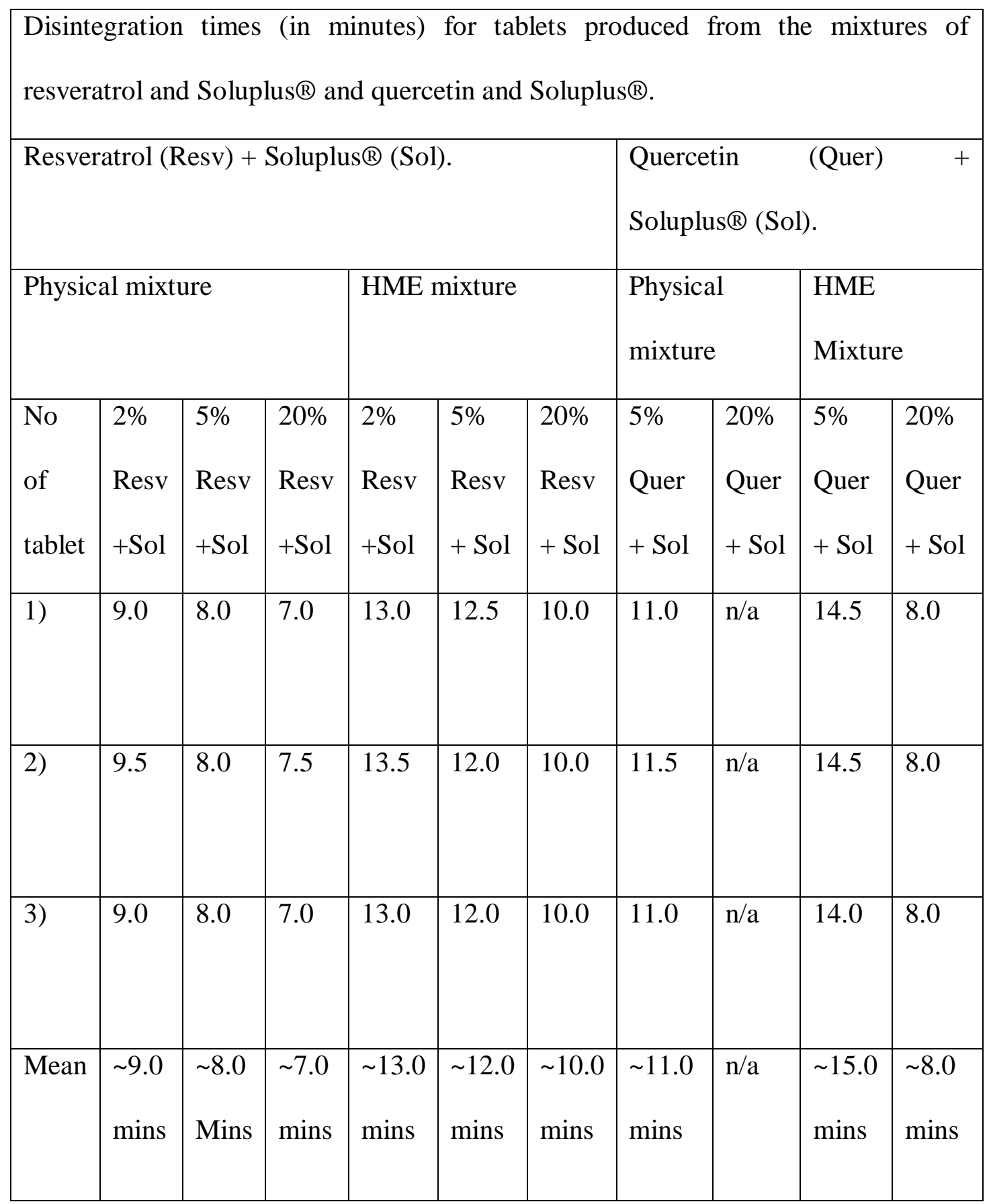

Table 11: Data obtained for the mean disintegration times $( \pm$ SD) of the tablets produced by the tablets obtained from the physical mixtures and HME mixtures of resveratrol and Soluplus ${ }^{\circledR}$ and quercetin and Soluplus ${ }^{\circledR}$.

In table 11, the data obtained for the disintegration time of the tablets produced from the physical mixtures and HME mixtures of resveratrol and Soluplus ${ }^{\circledR}$ and quercetin 
and Soluplus ${ }^{\circledR}$ have been shown. The data was obtained by conducting the test in a Vanguard LIJ-3 Disintegration Tester using simulated intestinal fluid at a temperature of $37^{\circ} \mathrm{C}$ for 15 minutes. Three tablets produced from each type of mixture were tested and the data shown is the average time taken by the three tablets to disintegrate.

The disintegration test is an in-vitro test used in order to predict the time taken by a tablet to undergo the process of swelling, cracking and breaking in the fluid environment of the GIT in addition to releasing the active compound for absorption. As per the standards set by the United States Pharmacopoeia (USP), the tablet should not take more than 15 minutes to disintegrate during the test. From the data obtained, it is seen that the tablets produced by the HME mixtures take longer than the tablets produced from the physical mixture to undergo disintegration. Amongst the HME mixtures, the tablets produced from the mixture containing 5\% quercetin by weight and Soluplus ${ }^{\circledR}$ takes nearly 15 minutes to disintegrate. The tablets produced from the HME mixture containing $20 \%$ quercetin by weight takes the shortest time to disintegrate amongst all the tablets produced from the HME mixtures of resveratrol and Soluplus ${ }^{\circledR}$ and quercetin and Soluplus ${ }^{\circledR}$. Due to their brittle nature, the tablets produced by the physical mixtures of resveratrol have comparatively shorter disintegration times than the tablets produced by the physical mixture of quercetin and Soluplus®.

Interpretations and conclusion

The study involved the formulation of tablets produced from the HME mixtures and the physical mixtures of resveratrol and Soluplus ${ }^{\circledR}$ and quercetin and Soluplus ${ }^{\circledR}$ and a 
comparison of their characteristics by performing the standard analytical tests on a batch of tablets. The data obtained from the tests showed that the tablets produced by the physical mixture of resveratrol with Soluplus ${ }^{\circledR}$ were more brittle, more friable and had shorter disintegration times as compared to the tablets produced from the mixture of quercetin with Soluplus®. However, the formulation of the physical mixture containing $20 \%$ quercetin by weight and Soluplus® into tablets was not successful. Hence, no data could be obtained on this mixture.

The higher friability of the tablets produced from the mixture of resveratrol and Soluplus ${ }^{\circledR}$ shows that the tablets might undergo cracking, breaking, chipping or a loss of mass upon packaging, storage and transportation. The lower hardness values confirm the above finding and suggest that the tablets might not sustain the rigors of further processing. The reverse is true for the tablets produced from the HME mixtures of quercetin and Soluplus ${ }^{\circledR}$ as they have sufficient hardness and low friability values as observed. The disintegration test indicated the shorter disintegration times for the tablets produced from the physical mixtures of the polyphenol and Soluplus@. Additionally, the tablets produced from the HME mixture of $20 \%$ quercetin by weight and Soluplus@ has a short disintegration time which suggests faster rate of breaking and perhaps, faster rate of dissolution than the tablets produced from the other HME mixtures. Summarily, the tablets produced from the HME mixtures of both polyphenols in Soluplus ${ }^{\circledR}$ have better characteristics than the tablets produced from their corresponding physical mixtures in terms of adequate hardness, lower friability and acceptable disintegration times. 
Study 8

A study of the change in the properties of the hot-melt extruded mixtures under forced degradation at conditions of elevated temperature and humidity

Results

About $5 \mathrm{gm}$ of the samples of the stress-tested HME mixtures of $2 \%$ and $5 \%$ resveratrol by weight and Soluplus ${ }^{\circledR}$ and $5 \%$ and $20 \%$ by weight of quercetin and Soluplus ${ }^{\circledR}$ were filled into clean glass vials and placed in an oven maintained at $40^{\circ} \mathrm{C}$ under $75 \%$ relative humidity for a period of one month. At the end of this period, the samples were first dried, re-weighed and analyzed by HPLC. The chromatograms obtained as a result have been shown below.

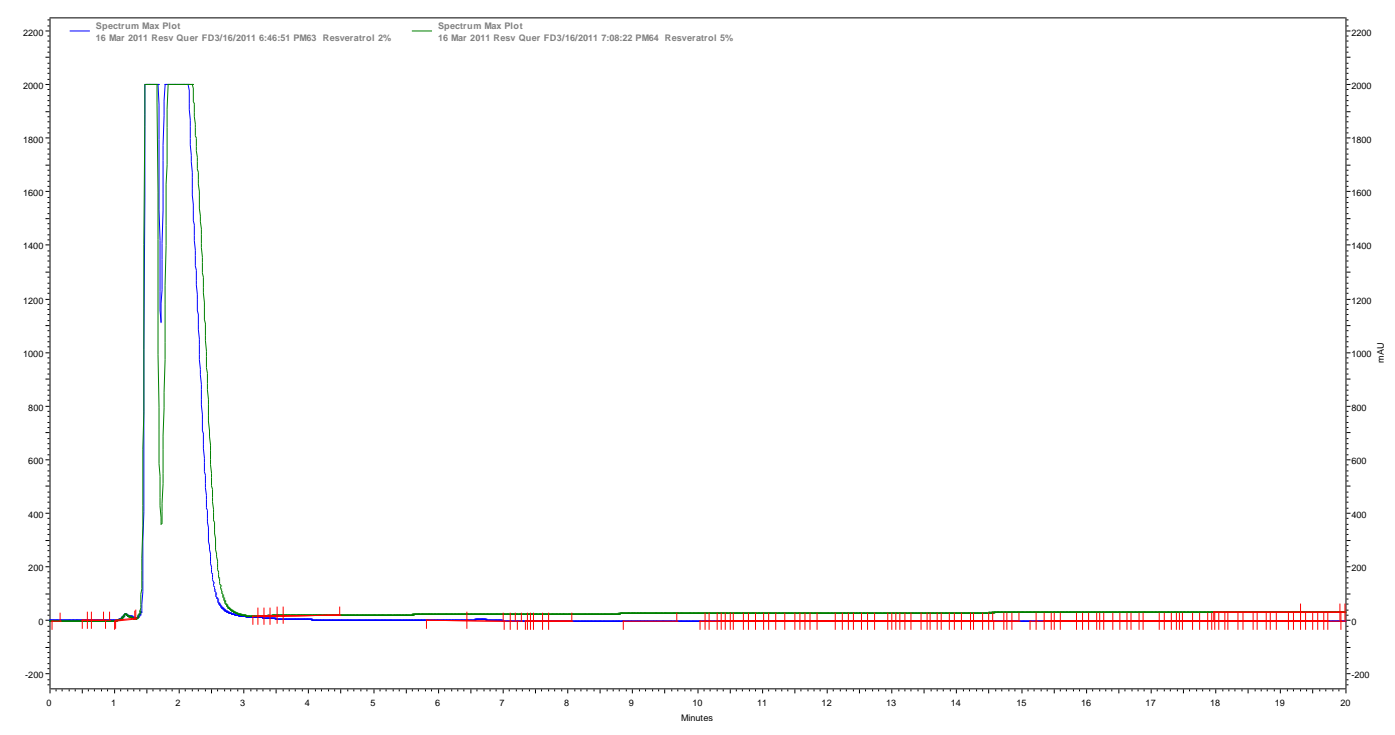

Figure 42: Multiple chromatogram view of the HME mixtures of $2 \%$ and $5 \%$ resveratrol by weight and Soluplus ${ }^{\circledR}$ after forced degradation.

A chromatogram was also obtained for the HME mixture containing $20 \%$ by weight of resveratrol and Soluplus ${ }^{\circledR}$ as shown in figure 46 


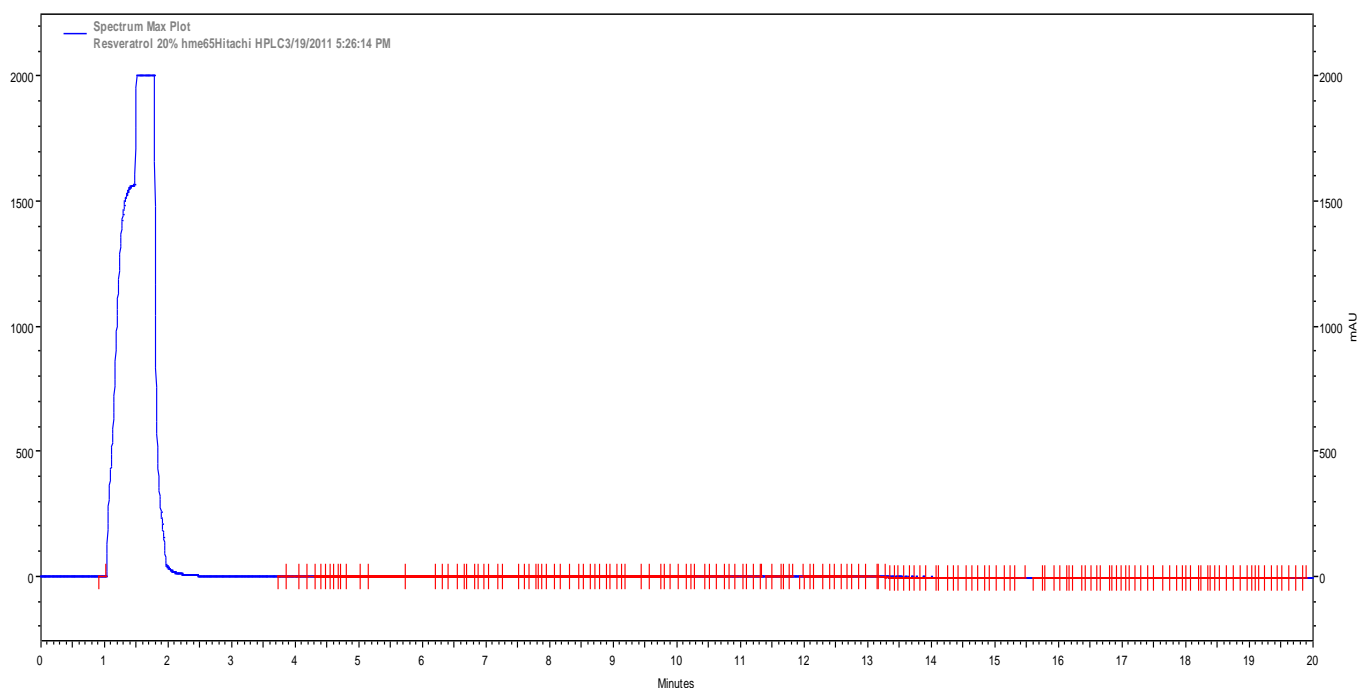

Figure 43: Chromatogram obtained for HME mixture containing 20\% resveratrol by weight and Soluplus ${ }^{\circledR}$ after forced degradation.

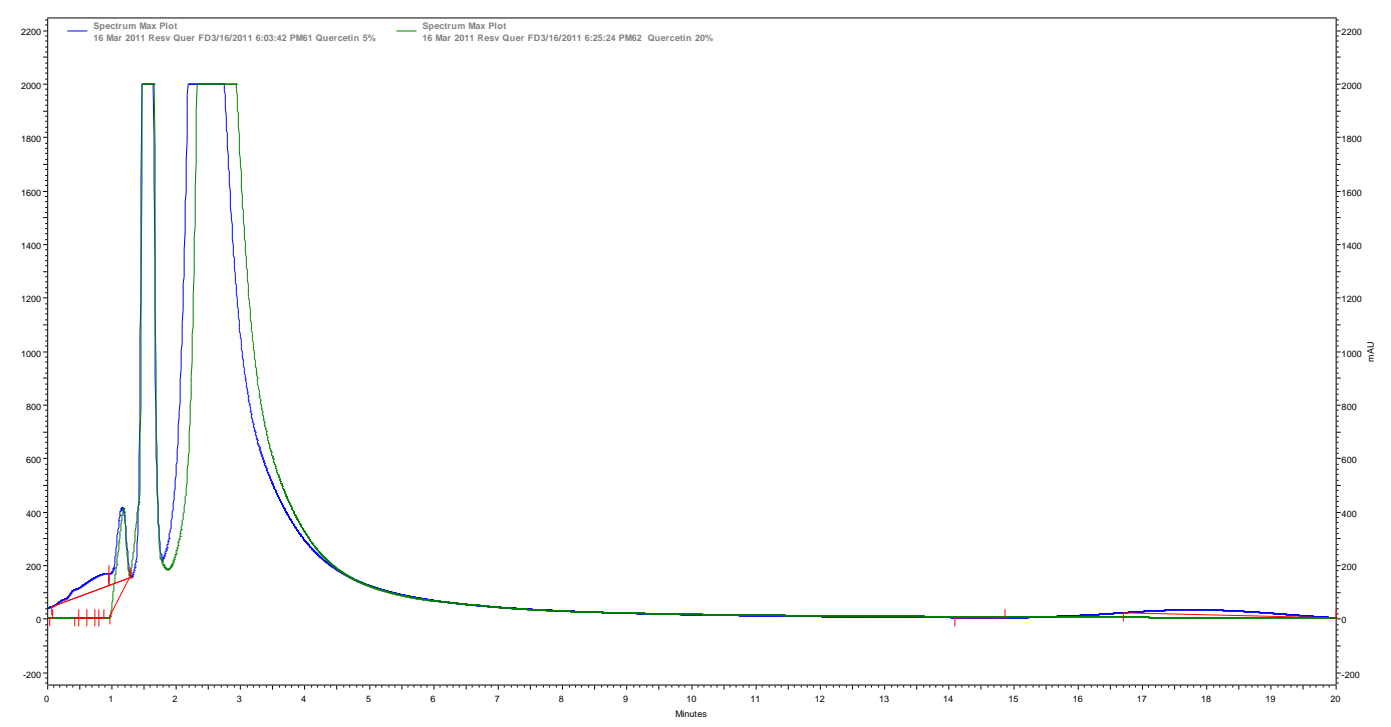

Figure 44: Multiple chromatogram view of the HME mixtures of $5 \%$ and $20 \%$ by weight of quercetin and Soluplus ${ }^{\circledR}$ after forced degradation.

The chromatogram obtained for each type of mixture was then compared to the chromatogram obtained for the same mixture before the degradation studies were 
carried out. The following figures show the chromatograms obtained on overlaying the chromatograms obtained in figures 42 and 44 with the chromatograms obtained on performing the content uniformity test for the HME mixtures of the polyphenols and Soluplus@.

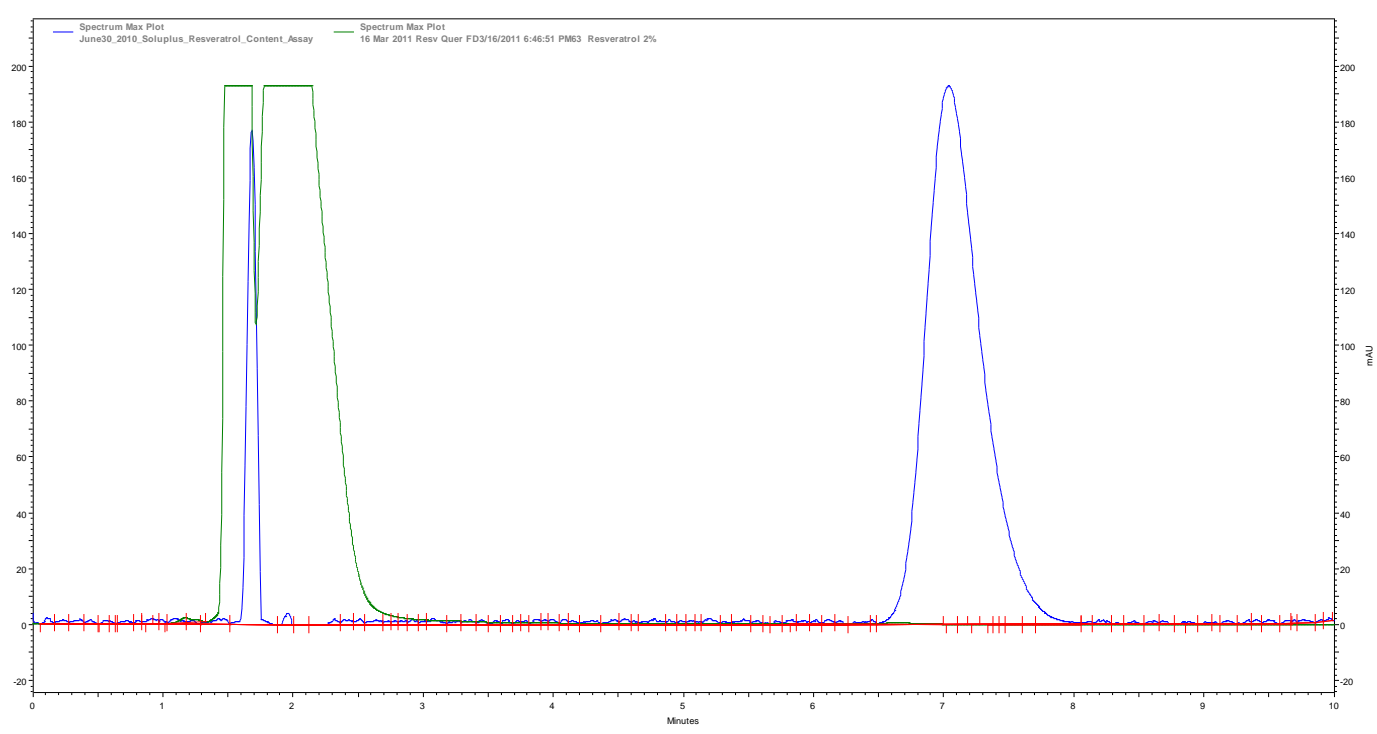

Figure 45: Multiple chromatogram view of $2 \%$ resveratrol by weight, HME with Soluplus $®$, before and after forced degradation. 


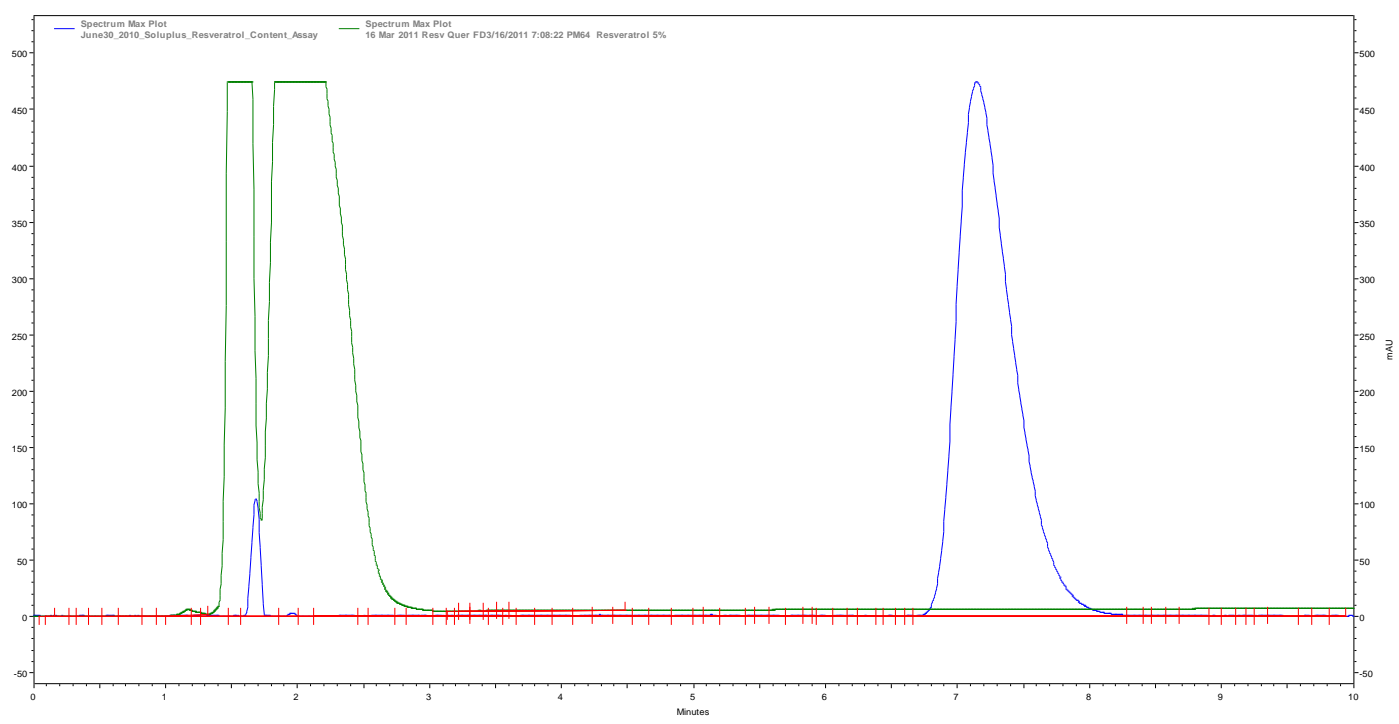

Figure 46: Multiple chromatogram view of 5\% resveratrol by weight, HME with Soluplus ${ }^{\circledR}$, before and after forced degradation.

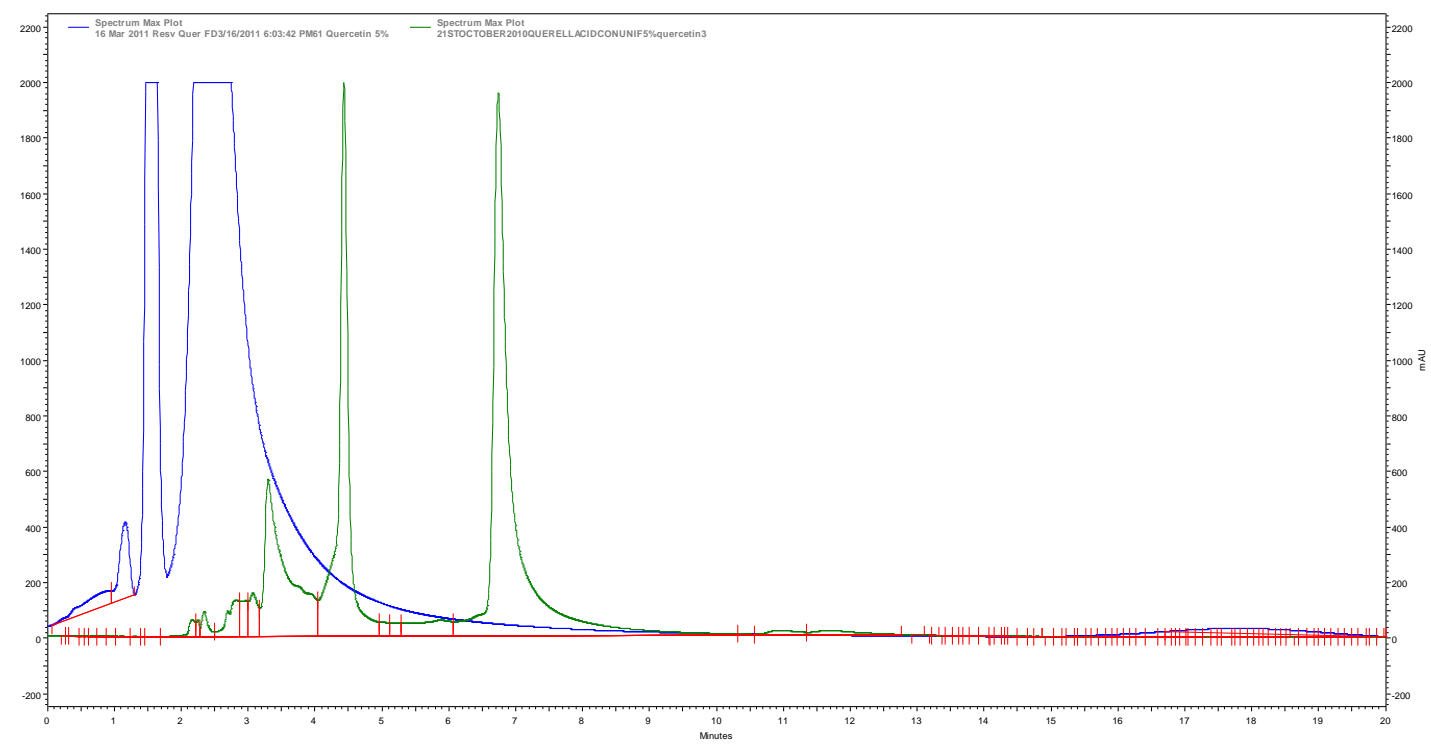

Figure 47: Multiple chromatogram view of $5 \%$ quercetin by weight, HME with Soluplus ${ }^{\circledR}$, before and after forced degradation. 


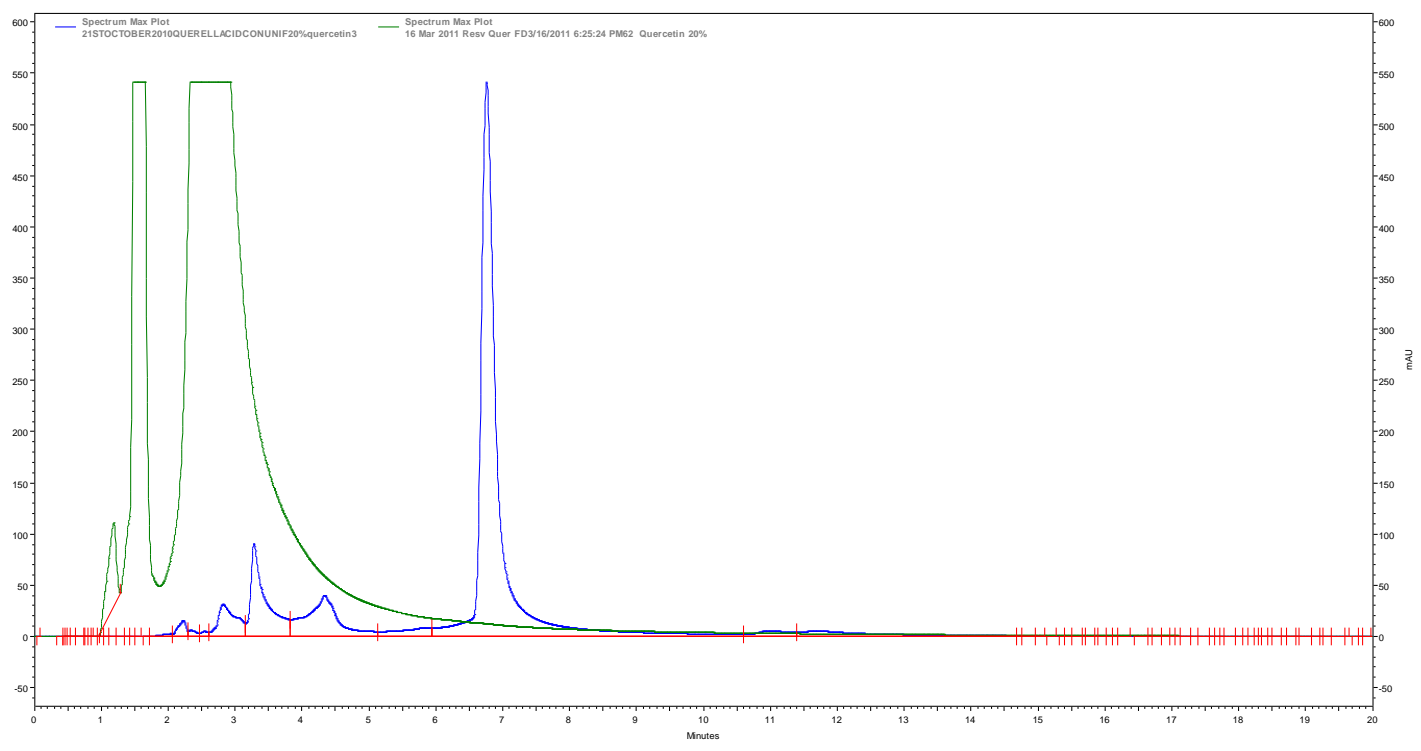

Figure 48: Multiple chromatogram view of $20 \%$ quercetin by weight, HME with Soluplus®, before and after forced degradation.

From the figures 45 and 46, it is observed that the peak value that was initially obtained in the case of the HME mixtures of resveratrol with a retention time between 6 and 7 minutes is not seen after forced degradation studies. Only a split peak is obtained with a retention time between 1 and 2 minutes. All the HME mixtures of resveratrol show the same trend in their respective chromatograms before and after forced degradation studies.

From the figures 47 and 48, similar observations can be made in the case of the HME mixtures containing quercetin. The split peaks obtained with a retention time between 2 and 4 minutes in the HME mixtures before degradation is now obtained with a retention time between 1 and 3 minutes. The other peak seen in the HME mixtures of quercetin before forced degradation with a retention time at close to 7 minutes is also not observed in the mixtures after forced degradation. 
Hence, the exposure of the HME mixtures to relative humidity of $75 \%$ and an elevated temperature of $40^{\circ} \mathrm{C}$ for one month has perhaps resulted in some amount of degradation of the mixtures resulting in, perhaps, the loss of the active polyphenol in each HME mixture. As a result of this degradation, the HPLC peaks characteristic of the polyphenols that were observed before degradation are now absent. This disappearance of such characteristic peaks from the mixture might be an indication of this degradation. Lastly, a slight shift in the peaks characteristic of Soluplus ${ }^{\circledR}$ within the HME mixtures containing quercetin might also provide some indication of this degradation. 


\section{Chapter 6}

\section{Conclusions}

Naturally occurring polyphenolic nutraceuticals such as resveratrol and quercetin have shown pharmacological activity against certain disease states. These nutraceuticals have very low solubility in water and have low bioavailability. In order to produce pharmaceutically active oral dosage forms of these nutraceuticals, techniques that improve their aqueous solubility and bioavailability have to be employed. Certain pharmaceutical manufacturing techniques such as spray drying, solvent evaporation and, more recently, hot-melt extrusion, have been used for the formulation of APIs with low aqueous solubility characteristics. However, in the case of resveratrol and quercetin, solvent evaporation did not produce any satisfactory results in terms of a viable dosage form instead giving rise to gummy masses that were not amenable to milling or tableting. Thus, hot-melt extrusion with a novel polymeric solubilizer, Soluplus ${ }^{\circledR}$, was employed. The extruded product was then analyzed for standard pharmaceutical properties such as its solubility, miscibility characteristics in organic solvents and in water, film formulations using these HME mixtures, FT-IR spectroscopy in order to determine the functional group characteristics of the HME mixtures, dissolution properties of these HME mixtures using hard gelatin capsules, a study of the thermal properties of the HME mixtures using DSC, a study of the properties of tablets produced from the HME mixtures and lastly, their content uniformity and forced degradation studies. 
The solubility and miscibility studies of the HME mixtures showed that melt extrusion with Soluplus ${ }^{\circledR}$ might perhaps have an effect of improving their solubility in water. The film formulation studies did not produce stable films for topical delivery of the polyphenols and showed signs of cracking and re-crystallization. The content uniformity studies showed that the HME mixtures remained uniform with respect to resveratrol and quercetin being used in different concentrations along with Soluplus®. The dissolution studies showed that smaller particles of the HME mixture were perhaps released at a more rapid rate than the larger particles from a hard gelatin capsule. From FT-IR spectroscopy of the HME mixtures, it was observed that there was an intimate interaction between resveratrol and Soluplus ${ }^{\circledR}$ and quercetin and Soluplus ${ }^{\circledR}$ during melt extrusion that might have produced certain changes at the molecular level in the polyphenols. Differential scanning calorimetry of the HME mixtures showed that the mixtures remain amorphous after extrusion and that quercetin had an apparent plasticization effect on Soluplus ${ }^{\circledR}$ when they are mixed. The tablet formulation studies might indicate towards quercetin tablets produced from the HME mixtures being harder, less friable and having suitable disintegration times as compared to the tablets produced from the HME mixtures of resveratrol. Lastly, the forced degradation studies may show that the HME mixtures undergo degradation under $40^{\circ} \mathrm{C}$ at $75 \%$ relative humidity and a loss in the concentration of the active polyphenol may occur as a result. 


\section{Bibliography}

(1) http://www.entheogens.org - Date accessed- 09/10

(2) Jingjun Huang, and Rodney J.Wignet, University of the Sciences in Philadelphia, Determination of drug and polymer miscibility and solid solubility for design of stable amorphous solid dispersions, American Pharmaceutical Review July/August 2009 18-26

(3) Stefano Petti, 'Sanarelli', Department of Public Health Sciences, "Sapienza', University of Rome, and Crispian Scully, "Eastman' Dental Institute, University College London, University of London, Polyphenols, oral health and disease: A review, Journal of Dentistry 37(2009) 413-423

(4) Farina A, Ferranti C, Marra C (2006). "An improved synthesis of resveratrol". Natural Product Research 20 (3): 247-52.

(5) Website: http://webbook.nist.gov/chemistry - Date accessed: 09/10

(6) "Pharma seeks genetic clues to healthy ageing". Reuters. 6 April 2010

(7) Bass TM, Weinkove D, Houthoofd K, Gems D, Partridge L (October 2007). "Effects of resveratrol on lifespan in Drosophila melanogaster and Caenorhabditis elegans". Mechanisms of Ageing and Development 128 (10): $546-52$

(8) Laura K. Stewart, Jeff L. Soileau, David Ribnicky, Zhong Q. Wang, Ilya Raskin, Alexander Poulev, Martin Majewski, William T. Cefalu, and Thomas W. Gettys (2008). "Quercetin transiently increases energy expenditure but persistently decreases circulating markers of inflammation in C57BL/6J mice fed a high-fat diet". Metabolism 57.

(9) J. Mark Davis, E. Angela Murphy, Martin D. Carmichael, and Ben Davis (2009), "Quercetin increases brain and muscle mitochondrial biogenesis and exercise tolerance", American Journal of Physiology; Regulatory, Integrative and Comparative Physiology 296. 
(10) http://webbook.nist.gov/chemistry. Date accessed: 10/10

(11) USDA Database for the Flavonoid Content of Selected Foods

Date accessed: $11 / 10$

(12) www.wikipedia.org/resveratrol. Date accessed: 03/10

(13) www.wikipedia.org/resveratrol. Date accessed: 03/10

(14) Völkel W, Colnot T, Csanády GA, Filser JG, Dekant W, "Metabolism and kinetics of bisphenol a in humans at low doses following oral administration," Chemical Research in Toxicology, 2002 Oct;15(10):1281-7.

(15) Makino T, Shimizu R, Kanemaru M, Suzuki Y, Moriwaki M, Mizukami H, Nagoya City University, "Enzymatically modified isoquercitrin, alphaoligoglucosyl quercetin 3-O-glucoside, is absorbed more easily than other quercetin glycosides or aglycone after oral administration in rats," Biological and Pharmaceutical Bulletin; 32(12):2034-40; 2009 December.

(16) Mao-Jung Lee, Joshua D. Lambert, Saileta Prabhu, Xiaofeng Meng, Hong Lu, Pius Maliakal, Chi-Tang Ho, and Chung S. Yang, "Delivery of Tea Polyphenols to the Oral Cavity by Green Tea Leaves and Black Tea Extract," Cancer Epidemiology, Biomarkers \& Prevention, Vol. 13, 132-137, January 2004.

(17) Leonarduzzi G, Testa G, Sottero B, Gamba P, Poli G, "Design and development of nanovehicle-based delivery systems for preventive or therapeutic supplementation with flavonoids," Current Medicinal Chemistry, 2010;17(1):74-95.

(18) Ghosh D, Ghosh S, Sarkar S, Ghosh A, Das N, Das Saha K, Mandal AK, "Quercetin in vesicular delivery systems: evaluation in combating arsenicinduced acute liver toxicity associated gene expression in rat model," Chemico-Biological Interactions. 2010 Jun 7; 186(1):61-71. Epub 2010 April 4.

(19) Hung CF, Lin YK, Huang ZR, Fang JY, "Delivery of resveratrol, a red wine polyphenol, from solutions and hydrogels via the skin," Biological and Pharmaceutical Bulletin 2008 May; 31(5):955-62. 
(20) http://www.walgreens.com/store/catalog/Vitamins/Resveratrol.

Date accessed: 10/10

(21) Shahani K, Swaminathan SK, Freeman D, Blum A, Ma L, Panyam J, "Injectable sustained release microparticles of curcumin: a new concept for cancer chemoprevention," Cancer Research. 2010 Jun 1; 70(11):4443-52.

(22) Crowley MM, Zhang F, Repka MA, Thumma S, Upadhye SB, Battu SK, McGinity JW, Martin C, "Pharmaceutical applications of hot-melt extrusion: part I," Drug Development and Industrial Pharmacy 2007 Sep; 33(9):909-26.

(23) Repka MA, Battu SK, Upadhye SB, Thumma S, Crowley MM, Zhang F, Martin C, McGinity JW, "Pharmaceutical applications of hot-melt extrusion: Part II,” Drug Development and Industrial Pharmacy 2007 Oct; 33(10):104357.

(24) Amidon, G.L., Lennernas, H., Shah, V.P. and Crison. J. R., "A theoretical basis for a biopharmaceutic drug classification: The correlation of in vitro drug product dissolution and in vivo bioavailability,"Pharmaceutical Research, $1995 ; 12: 413-420$.

(25) Jingjun Huang, and Rodney J.Wignet, "Determination of drug and polymer miscibility and solid solubility for design of stable amorphous solid dispersions," American Pharmaceutical Review, July/August 2009 18-26.

(26) R.J. Chokshi et al., Journal of Pharmaceutical Sciences, 2008, 97(6), 22862298.

(27) Breitenbach J, "Melt extrusion: from process to drug delivery technology," European Journal of Pharmaceutics and Biopharmaceutics, 2002, 54:107-117.

(28) Greenhalgh, D., Williams, A. C., Timmins, P., York, P., "Solubility parameters as predictors of miscibility in solid dispersions," Journal of Pharmaceutical Sciences, 88: 1182-1190.

(29) Skoog, Douglas A., F. James Holler and Timothy Nieman (1998). Principles of Instrumental Analysis (5 ed.). New York. pp. 805-808.

(30) Gavin P. Andrews, David S. Jones, Osama Abu Diak, Daniel N. Margetson, Mark S. McAllister, Hot-melt extrusion: an emerging drug delivery technology, Jan 2009, Pharmaceutical Technology Europe, Volume 21, Issue 1.

(31) www.basf.com. Date accessed: 06/10 
(32) www.pharma-ingredients.basf.com/Soluplus. Date accessed: 06/10

(33) A. S. Mujumdar (2007). Handbook of industrial drying. CRC Press. p. 710.

(34) Walter R. Niessen (2002). Combustion and incineration processes. CRC Press. p. 588.

(35) Charles Onwulata (2005). Encapsulated and powdered foods. CRC Press. p.66.

(36) http://en.wikipedia.org/wiki/File:SDXFamily1.JPG. Date accessed: 02/11.

(37) Priyanka Pandya, Surendra Gattani, Pankaj Jain, Lokesh Khirwal, Sanjay Surana, "Co-solvent Evaporation Method for Enhancement of Solubility and Dissolution Rate of Poorly Aqueous Soluble Drug Simvastatin: In vitro-In vivo evaluation," AAPS Pharmaceutical Sciences Technology; Vol. 9 Number 4, Dec 2008.

(38) http://www.rsc.org/Publishing/Journals/cc/News/B812111G Sasaki.asp

Date accessed: 02/11

(39) Hendrik Hardung, Dejan Djuric, and Shaukat Ali, "Combining HME and Solubilization- Soluplus ${ }^{\circledR}$ the solid solution,” Drug Delivery Technology; Vol. 10 Number 3, Page 20, April 2010.

(40) Williams RL, Adams WP, Poochikian G, Hauck WW, "Content uniformity and dose uniformity: current approaches, statistical analyses, and presentation of an alternative approach, with special reference to oral inhalation and nasal drug products," Pharmaceutical Research 2002 April; 19(4):359-66.

(41) Hua Zhang, and Lawrence X. Yu, "Dissolution Testing for Solid Oral Drug Products: Theoretical Considerations;Dissolution," American Pharmaceutical Review, Vol. 7, Issue 5, September/October 2004; 26-31.

(42) Sabine Pfeffer - Hennig \& Miriam Bellus, "Application of FT - Raman and FT - IR Spectroscopy in Pharmaceutical Development," American Pharmaceutical Review, Vol. 7, Issue 5, September/October 2004.

(43) A.T. Riga, L.M. Oberoil and K.S. Alexander, "Differential Scanning Calorimetry as a Pre-formulation Tool for Studying Eutectics Relevant in Pharmaceuticals," American Pharmaceutical Review, March/April 2004.

(44) Pungor, Erno (1995). A Practical Guide to Instrumental Analysis. Florida: Boca Raton. pp. 181-191. 
(45) Dean, John A. (1995). The Analytical Chemistry Handbook. New York: McGraw Hill, Inc.. pp. 15.1-15.5.

(46) Kibbe, A.H., ed. Handbook of Pharmaceutical Excipients. 3rd Edition ed. 2000, American Pharmaceutical Association \& Pharmaceutical Press: Washington, DC \& London, UK.

(47) USP <1217> chapter "Tablet breaking force" in USP 31 NF6, 20 January 2008.

(48) www.lib.njutcm.edu.cn/yaodian/jp/14data/Tablet_Friability_Test.

Date accessed: 03/11

(49) http://web.wilkes.edu/arthur.kibbe/lect16.html. Date accessed: 02/11

(50) Dan W. Reynolds, "Forced Degradation of Pharmaceuticals," American Pharmaceutical Review, May/June 2004.

(51) Robert M. Silverstein, Francis X. Webster, David J. Kiemle, " Spectrometric identification of organic compounds; Infra-red Spectrometry," $7^{\text {th }}$ Edition, 73110 . 